\title{
Propagação de singularidades para a equação da onda em variedades Lorentzianas: o teorema de Hörmander
}

\author{
Sandra María Zapata Yepes
}

\author{
DISSERTAÇÃO APRESENTADA \\ $\mathrm{AO}$ \\ INSTITUTO DE MATEMÁTICA E ESTATÍSTICA \\ DA \\ UNIVERSIDADE DE SÃO PAULO \\ PARA OBTENÇÃO DO GRAU \\ $\mathrm{DE}$ \\ MESTRE EM MATEMÁTICA APLICADA
}

Área de Concentração: Equações Diferenciais Parciais Orientador: Prof. Dr. Pedro Paulo Schirmer

Durante a elaboração deste trabalho o autor recebeu apoio financeiro da $\mathrm{CNPq}$ 


\section{PROPAGAÇÃO DE SINGULARIDADES PARA A EQUAÇÃO DA ONDA EM VARIEDADES LORENTZIANAS: O TEOREMA DE HÖRMANDER}

Este exemplar corresponde à redação final da tese de mestrado, devidamente corrigida e defendida por Sandra María Zapata Yepes aprovada pela comissão julgadora.

São Paulo, 23 de março de 2000.

Banca Examinadora:

- Prof.Dr. Pedro Paulo Schirmer - IME/USP

- Prof.Dr. Severino Toscano - IME/USP

- Prof.Dr. Milton da Costa Lopes Filho - IME/UNICAMP 
Aos meus pais. 


\section{Agradecimentos}

-Um agradecimento especial ao professor Dr. Pedro Paulo Schirmer, pela excelente orientação e dedicação.

-A meus amigos Jairo, Jose e Gladys por toda ajudả e presença em todos os momentos.

-A toda minha familia pelo apoio, amor e por suportar tanta saudade.

-A Adrilayne, Celso e Calixto pela grande colaboração na revisão ortográfica.

-A todos os amigos do IME e Enrique pela maravilhosa amizade e por compartilhar muitos bons momentos

-Ao CNPq pelo apoio financeiro. 


\section{Resumo}

A análise microlocal tem servido como ferramenta no estudo das equações diferenciais parciais lineares e não lineares.

Neste trabalho, são estudados alguns fatos gerais de operadores pseudo-diferenciais, operadores integrais de Fourier e construção da parametriz de um operador estritamente hiperbólico, aplicados na solução do problema de Cauchy para a equação da onda em variedades Lorentzianas. Também estudamos algumas caracterizações do conjunto frente de ondas permitindo uma análise mais completa das singularidades com a prova de uma versão simples do teorema devido a Hörmander sobre propagação de singularidades ao longo das bicaracterísticas nulas. 


\section{Abstract}

The microlocal analysis has served as a tool in the study of linear partial differential equations.

In this work, we have studed some general facts of pseudo-differential operators, Fourier integral operators and construction of the parametrix of the strictly hiperbolic operators, this applies to the solution of the Cauchy problem for the wave equation in Lorentzian Manifolds. Also we study some characteristics of the wave front set that permits a more complete analysis of the singularities with the proof of the simplest version of the Hörmander's theorem on propagation of singularities along the null bicharacteristics. 


\section{Sumário}

$\begin{array}{ll}\text { Introdução } & 6\end{array}$

1 Operadores Diferenciais e suas soluções fundamentais $\quad 10$

1.1 Algumas distribuições importantes . . . . . . . . . . . 10

1.2 Soluções Fundamentais . . . . . . . . . . . . . . . . . . . . 18

1.2.1 A solução fundamental do operador de Laplace em $\mathbb{R}^{n}$. . 18

1.2.2 A solução fundamental do operador de onda em $\mathbb{R}^{n+1} \ldots 19$

2 Cálculo dos operadores Pseudo-diferenciais $\quad 25$

2.1 Integrais Oscilatórias . . . . . . . . . . . . . . 25

2.2 Estimativas da fase estacionária: . . . . . . . . . . . . . 33

2.2 .1 Caso 1-dimensional: . . . . . . . . . . . . . . 35

2.2 .2 Fase estacionária em dimensões superiores: . . . . . . . . 38

2.3 Operadores Pseudo-diferenciais . . . . . . . . . . . 42

2.3 .1 Definições . . . . . . . . . . . . . . . . . . 42

2.3.2 Cálculo Pseudo-Diferencial . . . . . . . . . . . . . . 44

2.3.3 Continuidade em $L^{2}$ dos Operadores Pseudo-Diferenciais . 49

3 Conjunto Frente de Ondas 53

4 Operadores integrais de Fourier $\quad 69$

4.1 Álgebra de simplectomorfismos . . . . . . . . . . . . 69

4.2 Distribuições Lagrangeanas . . . . . . . . . . . . . . . 74

4.3 Continuidade dos operadores integrais de Fourier . . . . . 79 
5 Parametriz de um operador hiperbólico de segunda ordem $\quad 85$

5.1 Superfícies características, fluxo bicaracterístico . . . . . . . 85

5.1 .1 Superfícies características . . . . . . . . . 85

5.1.2 Método das bicaracterísticas: . . . . . . . . . . . . 89

5.2 Variedades Lorentzianas . . . . . . . . . . . . . . . . 91

5.3 Parametriz de um operador hiperbólico de segunda ordem . . . . 93

6 O teorema de propagação de singularidades 101

$\begin{array}{ll}\text { A o lema de Morse } & 104\end{array}$

$\begin{array}{ll}\text { B Subespaços lagrangeanos } & 106\end{array}$

$\begin{array}{ll}\text { C Propriedades da função beta } & 107\end{array}$

$\begin{array}{ll}\text { Referências Bibliográficas } & 107\end{array}$

$\begin{array}{ll}\text { Índice Remissivo } & 109\end{array}$ 


\section{Introdução}

O objetivo desta dissertação é estudar a equação da onda linear em variedades Lorentzianas $\left(M^{n+1}, g\right)$ dotada da métrica $g=g_{\alpha \beta} d x^{\alpha} d x^{\beta}$, isto é,

$$
\left\{\begin{array}{c}
P u=g^{\alpha \beta} \partial_{\alpha} \partial_{\beta} u+b^{\alpha} \partial_{\alpha} u+c u=0 \\
\left.u\right|_{\Sigma}=u_{0},\left.\quad \partial_{\nu} u\right|_{\Sigma}=u_{1}
\end{array}\right.
$$

onde $\Sigma \subset M^{n+1}$ é uma hiperfície de tipo espaço e $v \in(T \Sigma)^{\perp}$ é o vetor normal à hiperfície $\Sigma$. Como as estimativas apresentadas neste trabalho sã்o apenas locais, podemos assumir que $M^{n+1}$ é um aberto do espaço de Minkowski $\mathbb{R}^{n+1}$ e que $\Sigma$ seja a hiperfície $\{t=0\}$. O problema de obter estimativas globais para variedades Lorentzianas não compactas $M^{n+1}$ é muito mais difícil e foge ao escopo deste trabalho. Quando a métrica $g$ é a métrica de Minkowski $g=\operatorname{diag}(-1,1, \ldots, 1) \mathrm{em}$ $\mathbb{R}^{n+1}$, então é muito simples achar uma solução do problema de Cauchy usando a transformada de Fourier. De fato,

$$
\begin{gathered}
\partial_{t}^{2} f-\Delta f=0 \\
\left.f\right|_{t=0}=0, \quad \partial f /\left.\partial t\right|_{t=0}=u(x) \quad \in C_{0}^{\infty}\left(\mathbb{R}^{n}\right) .
\end{gathered}
$$

Vamos achar a solução de (1)-(2) com ajuda da transformada de Fourier em $x$,

$$
\hat{f}(x, t)=\int e^{-i y \cdot \xi} f(t, y) d y .
$$

Assumindo que é válida a diferenciação sob o sinal de integração, temos

$$
\begin{aligned}
\partial^{2} \hat{f} / \partial t^{2} & =\int \partial^{2} f(t, y) / \partial t^{2} e^{-i y \xi} d y=\int \Delta f e^{-i y \xi} d y \\
& =\int f \Delta\left(e^{-i y \xi}\right) d y=-|\xi|^{2} \int f e^{-i y \xi} d y=-|\xi|^{2} \hat{f}(t, \xi) .
\end{aligned}
$$


Assim,

$$
\partial^{2} \hat{f} / \partial t^{2}=-|\xi|^{2} \hat{f}(t . \xi)
$$

com

$$
\left.\hat{f}\right|_{t=0}=0, \quad \partial \hat{f} /\left.\partial t\right|_{t=0}=\hat{u}(\xi),
$$

onde $\hat{u}(\xi)$ é a transformada de Fourier de $u(x)$. De (3)-(4) temos

$$
\hat{f}(t, \xi)=\hat{u}(\xi) \frac{\operatorname{sen} t|\xi|}{|\xi|} \text {. }
$$

Pela fórmula inversa da transformada de Fourier temos

$$
\begin{aligned}
f(t, x) & =(2 \pi)^{-n} \int e^{i x \xi} \hat{f}(t, \xi) d \xi \\
& =(2 \pi)^{-n} \int e^{i x \xi} \hat{u}(\xi) \frac{e^{i t|\xi|}-e^{-i t|\xi|}}{2 i|\xi|} d \xi \\
& =(2 \pi)^{-n} \iint e^{i x \xi} e^{-i y \xi} u(y) d y \frac{e^{i t|\xi|}-e^{-i t|\xi|}}{2 i|\xi|} d \xi \\
& =(2 \pi)^{-n} \iint e^{i(x-y) \xi+i t|\xi|}(2 i|\xi|)^{-1} u(y) d y d \xi \\
& -(2 \pi)^{-n} \iint e^{i[(x-y) \xi-t|\xi|]}(2 i|\xi|)^{-1} u(y) d y d \xi \\
& =\sum \pm \frac{1}{(2 \pi)^{n}} \iint e^{i[(x-y) \xi \pm t|\xi|]} \frac{1}{2 i|\xi|} u(y) d y d \xi, \\
f(t, x) & =\sum \pm \frac{1}{(2 \pi)^{n}} \iint e^{i[(x-y) \xi \pm t|\xi|]} \frac{1}{2 i|\xi|} u(y) d y d \xi .
\end{aligned}
$$

Quando a métrica $g$ não é a métrica de curvatura constante, então o procedimento acima não pode ser aplicado e devemos desenvolver novos métodos. Mais especificamente gostaríamos de obter uma parametriz local para o problema de Cauchy e examinar suas propriedades de regularidade. Vamos mostrar que para todo ponto $x \in M^{n+1}$ e para todo inteiro $N$ podemos construir em uma vizinhança $U$ de $x$ um operador que tem a forma

$$
u=I_{a, \phi}+R_{N},
$$

onde $I_{a, \phi}=\int e^{i \phi} a$ é um operador integral de Fourier que descreve a parte singular da solução e $R_{N} \in L^{-\infty}$ é um resto regularizante desprezível para o qual temos

$$
R_{N}: H^{s} \mapsto H^{s+N}, \quad \forall s .
$$


O trabalho consta de duas partes. Na primeira delas examinamos em detalhe a construção clásica da parametriz. Se $P$ é um operador de ordem $m$ e $u, \phi \in C^{\infty}(\Omega)$ então

$$
P\left(e^{i \phi} a\right)=e^{i \phi} P\left(x, \partial_{x}+i \partial \phi\right) a=e^{i \phi}(p(x . \operatorname{grad} \phi) a+\mathcal{T} a+\mathcal{R}),
$$

onde $p$ é uma função homogênea de grau $m$ sobre o fibrado cotangente $T^{*}(\Omega)$, chamado de polinômio característico ou símbolo principal de $P, \mathcal{T}$ é um operador diferencial de ordem 1 dependendo de $\rho$ e $\mathrm{R}$ é um resto desprezível. Os zeros de $p$ em $T^{*}(\Omega) \backslash\{0\}$ são chamados de características. A teoria de Hamilton-Jacobi para a equação característica $p(x, \operatorname{grad} \phi)=0$ se baseia na análise de certas curvas nas superfícies de nível de $\phi$, chamadas de bicaracterísticas (ver subseção 5.1.2) que permitem descrever a solução da equação eikonal $p(x, \operatorname{grad} \phi)=0$. Para determinar o símbolo, o método consiste em substituir a amplitude $a(t, x, \xi)$ pela série $a_{j k l} \quad l=1 \ldots N$ e comparar termos com o mesmo grau de homogeneidade, obtendo a solução de

$$
(P(x, D)+\mathcal{T})\left(a_{j k 0}+a_{j k 1}+\ldots+a_{j k N}\right)=0
$$

Além disto estabelecemos estimativas de Sobolev para o resto. Finalmente, examinando a parte singular $I_{a, \phi}$, estabelecemos as propriedades de propagação de singularidades, provando o célebre teorema de Hörmander no caso particular de operadores hiperbólicos. Dado que os objetos geométricos associados com $P$, tal como as características, moram no fibrado cotangente de $\Omega$, é natural esperar que elas possam ser mais facilmente relacionadas ao conjunto frente de ondas (WF). Assim, o teorema do capítulo 6 afirma que as singularidades propagam-se ao longo das bicaracterísticas nulas.

No primeiro capítulo, serão estudadas algumas distribuições importantes e a solução fundamental para alguns operadores diferenciais. As principais referências são os livros de J. Hounie [4], L. Hörmander [\$] e P. Cordaro [11].

No segundo capítulo, serão estudadas: integrais oscilatórias, estimativas da fase estacionária e algumas propriedades dos operadores pseudo-diferenciais. As principais referências são os livros de J. Hounie [־], L. Hörmander [8], C. Sogge [1], L. Nirenberg [9] e M.Taylor [3].

No terceiro capítulo, serão estudadas generalidades sobre o conjunto frente de ondas. As principais referências são os trabalhos de L. Hörmander [8, 7, 2], C. 
Sogge [1], L. Nirenberg [9] e Shubin [10].

No quarto capítulo, serão estudados os operadores integrais de Fourier definidos com seu kernel nos espaços de distribuições Lagrangianas. As principais referências são os livros de C. Sogge [1] e F. Treves [3].

No quinto capítulo, será estuda a construção da parametriz como uma aplicação para o operador de onda, em dois casos: com coeficientes constantes e com coeficientes variáveis. As principais referências são os livros de F. Treves [3] e P.P. Schirmer [12].

No sexto - e último - capítulo será estudada uma versão simples do teorema de propagação de singularidades de Hörmander. As principais referências são os trabalhos de L. Nirenberg [9], L. Hörmander [6] e Shubin [10]. 


\section{Capítulo 1}

\section{Operadores Diferenciais e suas soluções fundamentais}

\subsection{Algumas distribuições importantes}

Definição 1.1 Uma distribuição num aberto $\Omega \subseteq \mathbb{R}^{n}$ é um funcional linear contínuo $u: C_{0}^{\infty}(\Omega) \rightarrow \mathbb{C}$, tal que para todo compacto $K \subseteq \Omega$ existe uma constante $C>0, \quad e \quad k \in \mathbb{Z}_{+}$com

$$
|u(\phi)| \leq C \sum_{|\alpha| \leq k} \sup \left|\partial^{\alpha} \phi\right| \quad \forall \phi \in C_{0}^{\infty}(K) .
$$

Denotamos por $\mathcal{D}^{\prime}(\Omega)$ o espaço das distribuições sobre $\Omega$. Se $k \in \mathbb{Z}_{+}$pode ser escolhido uniformemente para todo compacto $K \subseteq \Omega$, dizemos que $u$ tem ordem $\leq k$ e denotamos $u \in \mathcal{D}_{(k)}^{\prime}(\Omega)$.

Definição 1.2 Se $u \in \mathcal{D}^{\prime}(X)$, então o suporte singular de $u$, denotado por suppsing $(u)$, é o conjunto complementar do maior aberto onde $u \in C^{\infty}$.

Exemplos:

1. Delta de Dirac: $\delta \in \mathcal{D}^{\prime}(\Omega)$, de fato, para todo compacto $K \subseteq \Omega$, existe $C$ tal que

$$
|\delta(\phi)|=|\phi(0)| \leq C \sup |\phi| \quad \forall \phi \in C_{0}^{\infty}(K), \quad \text { suppsing }(\delta)=\{0\}
$$


2. Parte principal da integral de Cauchy $P\left(\frac{1}{x}\right)$. É uma renormalização da função não-integrável $\frac{1}{x} \notin L_{\text {Loc }}^{1}(\mathbb{R})$

$$
P\left(\frac{1}{x}\right): \phi \mapsto \lim _{\epsilon \rightarrow 0} \int_{|x| \geq \epsilon} \frac{1}{x} \phi(x) d x
$$

com $\phi \in \mathcal{S}(\mathbb{R})$. Vejamos que $P\left(\frac{1}{x}\right) \in \mathcal{D}_{(1)}^{\prime}(\mathbb{R})$. Sejam $a>0$, e $\phi \in$ $C_{0}^{\infty}([-a, a])$. Pelo teorema fundamental do cálculo

$$
\phi(x)=\phi(0)+\int_{0}^{x} \phi^{\prime}(t) d t=\phi(0)+x \int_{0}^{1} \phi^{\prime}(t x) d t .
$$

Seja $\chi(x)=\int_{0}^{1} \phi^{\prime}(t x) d t$ então

$$
\sup _{[-a, a]}|\chi| \leq \sup _{[-a, a]}\left|\phi^{\prime}\right| .
$$

Usando o fato que $x \mapsto \frac{1}{x}$ é uma função ímpar,

$$
\begin{aligned}
\int_{\epsilon \leq|x| \leq a} \frac{\phi(x)}{x} d x & =\int_{\epsilon \leq|x| \leq a} \frac{\phi(x)-\phi(0)}{x} d x+\phi(0) \underbrace{\int_{\epsilon \leq|x| \leq a} \frac{1}{x} d x=0}_{\epsilon \leq|x| \leq a} \\
& =\int \chi(x) d x
\end{aligned}
$$

e então eliminando a singularidade:

$$
\lim _{\epsilon \rightarrow 0} \int_{|x| \geq \epsilon} \frac{1}{x} \phi(x) d x=\int_{|x| \leq a} \chi(x) d x .
$$

Assim

$$
\left|P\left(\frac{1}{x}\right)(\phi)\right| \leq 2 a \sup _{[-a, a]}\left|\phi^{\prime}\right|, \quad \forall \phi \in C_{0}^{\infty}([-a, a])
$$

Definição 1.3 Se $u \in \mathcal{D}^{\prime}(\Omega)$ e se $\alpha \in \mathbb{Z}_{+}{ }^{n}$, definimos a derivada distribuicional

$$
\partial^{\alpha} u: C_{0}^{\infty}(\Omega) \mapsto \mathbb{C}
$$

$\operatorname{por}\left(\partial^{\alpha} u\right)(\phi)=(-1)^{|\alpha|} u\left(\partial^{\alpha} \phi\right)$.

Definição 1.4 Se $f \in C^{\infty}(\Omega)$ e $u \in \mathcal{D}^{\prime}(\Omega)$ definimos

$$
(f u)(\phi)=u(f \phi), \quad \forall \phi \in C_{0}^{\infty}(\Omega) .
$$




\section{Exemplos:}

1. (função de Heaviside)

$$
H(x)=\left\{\begin{array}{ccc}
1 & \text { se } \quad x>0 \\
0 & \text { se } \quad x \leq 0
\end{array} .\right.
$$

Vejamos que $H^{\prime}=\delta$ em $\mathcal{D}^{\prime}(\mathbb{R}) ;$ de fato, se $\phi \in C_{0}^{\infty}(\mathbb{R})$

$$
\begin{aligned}
H^{\prime}(\phi)=-H\left(\phi^{\prime}\right) & =-\int H(x) \phi^{\prime}(x) d x \\
& =-\int_{0}^{\infty} \phi^{\prime}(x) d x=\phi(0) .
\end{aligned}
$$

2. $(\log |x|)^{\prime}=P\left(\frac{1}{x}\right)$; de fato, $\log |x| \in L_{L o c}^{1}(\mathbb{R})$ e se $\phi \in C_{0}^{\infty}(\mathbb{R})$ então

$$
\begin{aligned}
(\log |x|)^{\prime}(\phi) & =-(\log |x|)\left(\phi^{\prime}\right)=-\int_{\mathbb{R}} \log |x| \phi^{\prime}(x) d x \\
& =-\lim _{\epsilon \rightarrow 0^{+}} \int_{|x| \geq \epsilon} \log |x| \phi^{\prime}(x) d x \\
& =-\lim _{\epsilon \rightarrow 0^{+}}\left\{\int_{\epsilon}^{\infty} \log (x) \phi^{\prime}(x) d x+\int_{-\infty}^{-\epsilon} \log (-x) \phi^{\prime}(x) d x\right\} \\
& =-\lim _{\epsilon \rightarrow 0^{+}}\left\{[\log x \phi(x)]_{\epsilon}^{\infty}-\int_{\epsilon}^{\infty} \frac{\phi(x)}{x} d x\right. \\
& \left.+[\log (-x) \phi(x)]_{-\infty}^{-\epsilon}-\int_{-\infty}^{-\epsilon} \frac{\phi(x)}{x} d x\right\} \\
& =\lim _{\epsilon \rightarrow 0^{+}}\left\{\log \epsilon\left[\phi(\epsilon)-[\phi(-\epsilon)]+\int_{|x| \geq \epsilon} \frac{\phi(x)}{x} d x\right\}\right. \\
& =P\left(\frac{1}{x}\right)(\phi) .
\end{aligned}
$$

3. $(x \log |x|)^{\prime \prime}=P\left(\frac{1}{x}\right)$; a prova é análoga ao (2.).

Uma classe importante de distribuições aparece como valores de fronteira de certas funções holomorfas. Para este fim, adotamos coordenadas complexas em $\mathbb{R}^{2}=\mathbb{C}, z=x+i y$ e definimos os operadores de Cauchy-Riemann:

$$
\frac{\partial}{\partial z}=\frac{1}{2}\left(\frac{\partial}{\partial x}-i \frac{\partial}{\partial y}\right), \quad \frac{\partial}{\partial \bar{z}}=\frac{1}{2}\left(\frac{\partial}{\partial x}+i \frac{\partial}{\partial y}\right)
$$


Definição 1.5 Se $\Omega \subseteq \mathbb{C}$ é um aberto, uma função $f \in C^{1}(\Omega)$ é holomorfa $\mathrm{em}$ $\Omega$ se $\frac{\partial f}{\partial \bar{z}}=0$. Denotamos o espaço das funções holomorfas em $\Omega$ por $\mathcal{O}(\Omega)$.

Lema 1.1 Se $\Omega \subseteq \mathbb{C}$ é um aberto convexo e $f \in \mathcal{O}(\Omega)$ então existe $g \in \mathcal{O}(\Omega)$ tal que $g^{\prime}=f$, isto é, $\frac{\partial g}{\partial z}=f$.

Prova: Se $f \in \mathcal{O}(\Omega), w=f d z$ então

$$
d w=d f \wedge d z=\left(\frac{\partial f}{\partial z} d z+\frac{\partial f}{\partial \bar{z}} d \bar{z}\right) \wedge d z
$$

como $\frac{\partial f}{\partial \bar{z}}=0$ então $d w=0$ e pelo Lema de Poincaré, existe uma função $g \in C^{\infty}(\Omega)$ tal que $d g=w$, isto é, $d g=\frac{\partial g}{\partial z} d z+\frac{\partial g}{\partial \bar{z}} d \bar{z}=f d z$ então $\frac{\partial g}{\partial z}=f$ e $\frac{\partial g}{\partial \bar{z}}=0$ portanto $g \in \mathcal{O}(\Omega)$.

\section{Lema 1.2 (Teorema de Gauss no plano complexo)}

Sejam $\Omega \subseteq \mathbb{C}$ um aberto conexo e limitado com fronteira $\partial \Omega$ de classe $C^{1}$ orientada positivamente, $\bar{\Omega} \subseteq U$ e $\phi \in C_{0}^{1}(U)$. Então

$$
\int_{\Omega} \frac{\partial \phi}{\partial \bar{z}} d x d y=\frac{1}{2 i} \int_{\partial \Omega} \phi
$$

Prova: Tomando $f=\phi+i \phi \operatorname{com} \operatorname{div} f=2 \frac{\partial \phi}{\partial \bar{z}}$ e

$s$ o comprimento de arco, $\partial \Omega=(x(s), y(s)), \vec{\eta}=\left(-\frac{d y}{d s}, \frac{d x}{d s}\right)$ (normal exterior)

$f . \vec{\eta} d s=\left(-\phi \frac{d y}{d s}+i \phi \frac{d x}{d s}\right) d s$.

Pela fórmula de Gauss-Green sabemos que

$$
\int_{\Omega} \operatorname{divf}=-\int_{\partial \Omega} f \cdot \vec{\eta} d s
$$

Então temos que

$$
2 \int_{\Omega} \frac{\partial \phi}{\partial \bar{z}} d x d y=\int_{\Omega} \operatorname{divf}=-\int_{\partial \Omega}\left(-\phi \frac{d y}{d s}+i \phi \frac{d x}{d s}\right) d s=-i \int_{\partial \Omega} \phi d(x+i y) .
$$

Teorema 1.1 (Valor de fronteira de funções holomorfas)

Sejam uma faixa complexa de largura $\delta>0$ sobre um intervalo real $I=[a, b]$, 
$U=\{z=x+i y, \Re z \in[a, b],|\Im z|<\delta\}$ e $f \in \mathcal{O}(U)$ uma função holomorfa satisfazendo

$$
|f(z)| \leq \frac{C}{|\Im z|^{N}}, \quad z \in U .
$$

Então para cada $\phi \in C_{0}^{\infty}(I)$ existem os limites

$$
\lim _{y \rightarrow 0^{ \pm}} \int_{\mathbb{R}} f(x+i y) \phi(x) d x .
$$

Os funcionais $C_{0}^{\infty}(I) \mapsto \mathbb{C}$ definidos por

$$
\phi \mapsto \lim _{y \rightarrow 0^{ \pm}} \int_{\mathbb{R}} f(x+i y) \phi(x) d x
$$

definem distribuições sobre $I$, denominadas valores de fronteira de $f$ e denotadas por $f(x \pm i 0)^{1}$.

Antes de demonstrar este teorema, apresentamos um corolário importante:

Corolário 1.1 (Relações de Sokhotskii)

Os valores de fronteira da função complexa $f(z)=\frac{1}{z}$ são as distribuições

$$
\frac{1}{x \pm i 0}=P\left(\frac{1}{x}\right) \mp i \pi \delta(x)
$$

e satisfazem

$$
\frac{1}{x+i 0}-\frac{1}{x-i 0}=-2 i \pi \delta(x) \text {. }
$$

Vamos fazer duas provas para o teorema 1.1, a primeira será usada na prova do corolário de Sokhotskii a seguir e a segunda será usada num teorema mais adiante.

Prova do teorema 1.1: Vamos considerar $0<\Im(z)<\delta$ :

$$
\Gamma(y)=\int f(x+i y) \phi(x) d x .
$$

Neste caso

$$
\Gamma^{\prime}(y)=\int \partial_{y}\{f(x+i y)\} \phi(x) d x .
$$

Como $\partial_{y} f=i \partial_{x} f$ então

$$
\Gamma^{\prime}(y)=-i \int f(x+i y) \phi^{\prime}(x) d x .
$$

\footnotetext{
${ }^{1}$ Às vezes denotadas também por $b^{ \pm}(f) \in \mathcal{D}^{\prime}(\mathcal{I})$
} 
Por indução

$$
\Gamma^{(k)}(y)=(-i)^{k} \int f(x+i y) \phi^{(k)}(x) d x
$$

Assim

$$
\left|\Gamma^{(k)}(y)\right| \leq \int\left|f(x+i y) \phi^{(k)}(x)\right| d x \leq \frac{C_{k}}{y^{N}} .
$$

Logo $\Gamma \in C^{\infty}([0, \delta))$ portanto existe o limite

$$
\lim _{y \rightarrow 0^{+}} \int f(x+i y) \phi(x) d x=\Gamma(0) .
$$

Pelo lema 1.1 existe $g \in \mathcal{O}(I+i(0, \delta)) \cap C(I+i[0, \delta))$ tal que $g^{(N+1)}=f$. Então

$$
\begin{aligned}
\int f(x+i y) \phi(x) d x & =\int \partial_{x}^{\mathrm{N}+1}\{g(x+i y)\} \phi(x) d x \\
& =(-1)^{N+1} \int g(x+i y) \phi^{(N+1)}(x) d x
\end{aligned}
$$

e portanto

$$
b(f)(\phi)=(-1)^{N+1} \int g(x) \phi^{(N+1)}(x) d x .
$$

Consequentemente, $b(f)=g_{0}^{(N+1)}$ onde $g_{0}=\left.g\right|_{I+i\{0\}}$.

Prova do corolário de Sokhotskii: Sejam $f(z)=\frac{1}{z}, \Im z>0$ e $g \in \mathcal{O}(\{\Im z>$ $0\}) \cap C\{\Im z \geq 0\}) \operatorname{com} g^{(2)}(z)=\frac{1}{z}$. Assim, $g(z)=z \log (z)-z \operatorname{com} \log (z)=$ $\log |z|+\operatorname{iarg}(z)$

$$
g_{0}(x)=\left\{\begin{array}{ccc}
x \log (x)-x & \text { se } & x>0 \\
0 & \text { se } & x=0 \\
x(\log |x|+i \pi)-x & \text { se } & x<0
\end{array}\right.
$$

isto é, $g_{0}(x)=x \log |x|-x+i x \pi H(-x)$. Pelos exemplos 1.) e 3.) temos que

$$
b^{+}\left(\frac{1}{z}\right)=g_{0}^{\prime \prime}(x)=P\left(\frac{1}{x}\right)-i \pi \delta(x) .
$$

Analogamente, para $\Im z<0$

$$
b^{-}\left(\frac{1}{z}\right)=g_{0}^{\prime \prime}(x)=P\left(\frac{1}{x}\right)+i \pi \delta(x)
$$

e portanto

$$
b^{+}\left(\frac{1}{z}\right)-b^{-}\left(\frac{1}{z}\right)=-2 i \pi \delta(x)
$$


A outra prova do teorema 1.1: Para $\phi \in C_{0}^{N+1}(I)$

$$
\Phi(x, y)=\sum_{j \leq N} \phi^{(j)} \frac{(i y)^{j}}{j !}
$$

é a N-ésima soma parcial da expansão de Taylor em $y$. Então $\Phi(x, 0)=\phi(x)$ e

$$
2 \frac{\partial \Phi}{\partial \bar{z}}=\left(\frac{\partial}{\partial x}+\frac{\partial}{\partial y}\right) \Phi=\phi^{(N+1)}(x) \frac{(i y)^{N}}{N !}
$$

fixando $Y \operatorname{com} 0<Y<\gamma$. Se $0<y<\gamma-Y$ e aplicando o lema 1.2 a $\Phi(z) f(z+i y)$ com $0<\Im z<Y$ obtemos

$$
2 \int_{\Omega} \frac{(\partial \Phi(z) f(z+i y))}{\partial \bar{z}} d \lambda(z)=-i \int_{\partial \Omega} \Phi(z) f(z+i y) d z
$$

Como $f$ é holomorfa e pelo teorema de Stokes temos que

$2 i \iint_{0<\Im z<Y} f(z+i y) \frac{\partial \Phi(z)}{\partial \bar{z}} d \lambda(z)=\int \Phi(x, 0) f(x+i y) d x-\int \Phi(x, Y) f(x+i Y+i y) d x$,

onde $d \lambda$ é a medida de Lebesgue em $\mathbb{C}$. De (1.2) com $z=x+i t Y, \quad 0<t<1$ temos que

$$
\begin{aligned}
\int f(x+i y) \phi(x) d x & =\int \Phi(x, Y) f(x+i Y+i y) d x \\
& +\iint_{0}^{1} f(x+i t Y+i y) \phi^{(N+1)}(x) \frac{(i Y)^{N+1} t^{N}}{N !}
\end{aligned}
$$

Existe o limite uniforme para o integrando na dupla integral com $y \rightarrow 0$ e portanto

$$
\begin{aligned}
& \int f(x+i y) \phi(x) d x \rightarrow \\
& \int \Phi(x, Y) f(x+i Y) d x+\iint_{0}^{1} f(x+i t Y) \phi^{(N+1)}(x) \frac{(i Y)^{N+1} t^{N}}{N !} d x d t
\end{aligned}
$$

Definição 1.6 Uma distribuição $u \in \mathbb{R}^{n} \backslash\{0\}$ é chamada homogênea de grau a se

$<u, \phi>=t^{a}<u, \phi_{t}>\quad$ com $\phi_{t}(x)=t^{n} \phi(t x), \quad$ se $\phi \in C_{0}^{\infty}\left(\mathbb{R}^{n} \backslash\{0\}\right), t>0$ 
Se $a$ é um número complexo com $\Re a>-1$ então as funções sobre $\mathbb{R}$

$$
x_{+}^{a}=\left\{\begin{array}{ccc}
x^{a} & \text { se } & x>0 \\
0 & \text { se } & x \leq 0
\end{array} \quad \text { e } x_{-}^{a}=\left\{\begin{array}{ccc}
0 & \text { se } & x>0 \\
|x|^{a} & \text { se } & x \leq 0
\end{array}\right.\right.
$$

são localmente integráveis e cada uma define uma distribuição.

Propriedades:

1. $x x_{+}^{a}=x_{+}^{a+1}$ se $\Re a>-1$

2. $\frac{d}{d x} x_{+}^{a}=a x_{+}^{a-1}$ se $\Re a>0$

3. $<x_{-}^{a}, \phi>=<x_{+}^{a}, \phi(-x)>$

4.

$$
(x \pm i 0)^{a}=r^{a} e^{ \pm i a \theta}=x_{+}^{a}+e^{ \pm \pi i a} x_{-}^{a} .
$$

De fato, pelo teorema 1.1 (valor de fronteira) a função $z^{a}$, definida em $\mathbb{C} \backslash \mathbb{R}$ _ como $e^{a \log z}$, onde $\log z$ é real se $z \in \mathbb{R}_{+}$, tem valor de fronteira $(x \pm i 0)^{a}$ sobre o eixo real dos meios planos superior e inferior.

Seja $\Gamma(a)=\int_{0}^{\infty} x^{a-1} e^{-x} d x, \quad \Re a>0$, então $\Gamma(a+1)=a \Gamma(a)$ se $\Re a>0$. Definimos

$$
\chi_{+}^{a}=x_{+}^{a} \Gamma(a+1), \quad \Re a>-1,
$$

que é uma função analítica.

Teorema 1.2 Se $u \in \mathcal{D}^{\prime}\left(\mathbb{R}^{n} \backslash\{0\}\right)$ é homogênea de grau a que não é $\leq-n$, então u tem uma única extensão $u^{\prime} \in \mathcal{D}^{\prime}\left(\mathbb{R}^{n}\right)$ a qual é homogênea de grau a. Se $P$ é um polinômio homogêneo então $(P u)^{\prime}=P u^{\prime}$, e se $a \neq 1-n$ então $\left(\partial_{j} u\right)^{\prime}=\partial_{j} u^{\prime}$. A aplicação

$$
\mathcal{D}^{\prime}\left(\mathbb{R}^{n} \backslash\{0\}\right) \ni u \mapsto u^{\prime} \in \mathcal{D}^{\prime}\left(\mathbb{R}^{n}\right)
$$

é contínua.

Prova: Vide (8), capítulo III, $\oint(3.2)$ 


\subsection{Soluções Fundamentais}

Definição 1.7 Uma solução fundamental para o operador diferencial $P(D)=$ $\sum a_{\alpha} D^{\alpha}$ é uma distribuição $E \in \mathcal{D}^{\prime}$ tal que

$$
P(D) E=\delta
$$

O motivo para estudar a solução fundamental é que se definimos $u=E * f$ onde $f \in C_{0}^{\infty}$, então

$$
P(D) u=P(D)(E * f)=P(D) E * f=\delta * f=f .
$$

Assim, se pudermos achar uma solução fundamental, temos um teorema de existência para todas as equações diferencias parciais $P(D) u=f$, onde $f \in C_{0}^{\infty}$. Se pudermos achar uma expressão para $E$, temos uma representação explícita da solução, $u=E * f$.

\subsubsection{A solução fundamental do operador de Laplace em $\mathbb{R}^{n}$}

Teorema 1.3 Considere a função $E \in L_{l o c}^{1}\left(\mathbb{R}^{n}\right)$ definida por ${ }^{2}$ :

$$
E(x)=\left\{\begin{array}{cl}
-\frac{1}{(n-2) \omega_{n}} \frac{1}{\mid x^{n-2}}, & \text { se } n \geq 3 \\
\frac{1}{2 \pi} \log |x|, & \text { se } n=2 .
\end{array}\right.
$$

Prova-se que existem as derivadas parciais no sentido fraco, isto é,

$$
\frac{\partial E}{\partial x_{j}}=\frac{x_{j}}{\omega_{n}|x|^{n}} \in L_{l o c}^{1}\left(\mathbb{R}^{n}\right)
$$

e, além disto, E é a solução fundamental do operador de Laplace, isto é,

$$
\Delta E=\delta \quad \text { em } \quad \mathbb{R}^{n}
$$

Prova: Sabemos que, em coordenadas polares,

$$
\int_{\mathbb{R}^{n}} f(x) d x=\int_{s^{n-1}} \int_{0}^{\infty} f(r w) r^{n-1} d r d s(w) .
$$

\footnotetext{
${ }^{2}$ Aqui $\omega_{n}=\frac{2 \pi^{n / 2}}{\Gamma(n / 2)}$ é a área da esfera unitária em $\mathbb{R}^{n}$.
} 
Se $f(x)=|x|^{a}$ então $f \in L_{\text {loc }}^{1}\left(\mathbb{R}^{n}\right)$ se e somente se $a>-n$. Também sabemos que $\log |x| \in L_{\text {loc }}^{1}\left(\mathbb{R}^{2}\right)$. Temos que

$$
\begin{aligned}
\frac{\partial E}{\partial x_{j}}(\phi) & =-E \frac{\partial \phi}{\partial x_{j}}=-\lim _{\epsilon \rightarrow 0} \int_{|x|>\epsilon} E(x) \frac{\partial \phi}{\partial x_{j}}(x) d x \\
& =\int \frac{\partial E}{\partial x_{j}}(\phi)(x) d x+\lim _{\epsilon \rightarrow 0} \int_{|x|=\epsilon} E(x) \phi(x) \frac{\partial x_{j}}{\partial|x|}(x) d s(x) .
\end{aligned}
$$

Aplicando o teorema da divergência para $F(x)=(0,0, \ldots, E(x) \phi(x), 0, . .0)$ e $\vec{n}=$ $\frac{x}{|x|}$ temos, para $n \geq 3$

$\left|\int_{|x|=\epsilon} E(x) \phi(x) \frac{\partial x_{j}}{\partial|x|}(x) d s(x)\right| \leq$ Const $\frac{1}{\epsilon^{n-2}} \int_{|x|=\epsilon} d s(x)=O(\epsilon) \rightarrow 0 \quad$ com $\quad \epsilon \rightarrow 0$.

Para $n=2$

$$
\left|\int_{|x|=\epsilon} E(x) \phi(x) \frac{\partial x_{j}}{\partial|x|}(x) d s(x)\right| \leq O\left(\epsilon \log \frac{1}{\epsilon}\right) \rightarrow 0
$$

Finalmente

$(\Delta E)(\phi)=E(\Delta \phi)=\lim _{\epsilon \rightarrow 0} \int_{|x|>\epsilon} E(x) \Delta \phi(x) d x=\lim _{\epsilon \rightarrow 0} \int_{|x|>\epsilon}\{E(x) \Delta \phi(x)-\phi \Delta E(x)\} d x$.

Como $\Delta E=0$ no complementar da origem e $\operatorname{div}\{E g r a d \phi-\phi g r a d E\}=E \Delta \phi-$ $\phi \Delta E$, temos que

$$
\begin{aligned}
(\Delta E)(\phi) & =\lim _{\epsilon \rightarrow 0} \int_{|x|=\epsilon}<\phi \operatorname{gradE}-\operatorname{Egrad} \phi, \frac{x}{|x|}>d s(x) \\
& =\lim _{\epsilon \rightarrow 0} \int_{|x|=\epsilon}<\phi \operatorname{grad} E, \frac{x}{|x|}>d s(x) \\
& =\lim _{\epsilon \rightarrow 0} \int_{|x|=\epsilon} \frac{1}{\omega_{n}} \phi(x) \frac{1}{|x|^{n-1}} d(x)=\phi(0) .
\end{aligned}
$$

\subsubsection{A solução fundamental do operador de onda em $\mathbb{R}^{n+1}$}

Seja $A$ uma forma quadrática simétrica real e não singular em $\mathbb{R}^{n+1}$, isto é,

$$
A(x)=\sum a_{j k} x_{j} x_{k}, \quad \text { com } \frac{\partial A}{\partial x} \neq 0, \forall x \neq 0 .
$$

Se $\left(b^{j k}\right)=\left(a_{j k}\right)^{-1}$ é a matriz inversa, associamos à forma $A$ um operador diferencial $P(\partial)$ definido por:

$$
P(\partial)=\sum b^{j k} \partial_{j} \partial_{k}
$$


Suponhamos que $A$ seja uma forma quadrática Lorentziana, isto é, a restrição de $A$ a um subespaço n-dimensional $\mathbb{R}^{n} \subset \mathbb{R}^{n+1}$ é positiva-definida e a restrição ao espaço ortogonal uni-dimensional $\left(\mathbb{R}^{n}\right)^{\perp}$ é negativa. Neste caso, dizemos que o operador associado $P(\partial)$ é um operador de onda. Quando $A$ é a matriz

$$
\mathbb{A}=\left(\begin{array}{cccc}
-1 & 0 & 0 & \ldots \\
0 & 1 & 0 & \ldots \\
\vdots & \vdots & \ddots & \ldots \\
0 & 0 & \ldots & 1
\end{array}\right)
$$

o operador $P(\partial)$ coincide com o operador D' Alembertiano:

$$
P(\partial)=-\square=-\partial_{t}^{2}+\Delta_{\mathbb{R}^{n}}
$$

Vamos mostrar um teorema geral que permite determinar a solução fundamental do operador diferencial $P(\partial)$.

Teorema 1.4 Seja A uma forma quadrática Lorentziana em $\mathbb{R}^{n+1}$ e $P(\partial)=$ $\sum b^{j k} \partial_{j} \partial_{k}$ o operador diferencial associado à forma quadrática A. Se $\omega_{n+1}$ é a área da esfera unitária em $\mathbb{R}^{n+1}$ e $n \geq 2$, então

$$
\begin{gathered}
P(\partial)\left[\frac{(A \pm i 0)^{(1-n) / 2}}{(1-n) \omega_{n+1}|\operatorname{det} A|^{-1 / 2} e^{\mp \pi / 2}}\right]=\delta_{0} \\
P(\partial)\left[\frac{A^{*} \chi_{+}^{(1-n) / 2}}{+4 \pi^{(n-1) / 2} \sin (\pi n / 2)|\operatorname{det} A|^{-1 / 2}}\right]=\delta_{0}
\end{gathered}
$$

$e$

$$
P(\partial)\left[\frac{A^{*} \chi_{-}^{(1-n) / 2}}{-4 \pi^{(n-1) / 2}|\operatorname{det} A|^{-1 / 2}}\right]=\delta_{0}
$$

Para provar (1.7) precisamos do seguinte lema.

Lema 1.3 Sejam $F$ uma função $C^{\infty}(X \times J)$, onde $X \subset \mathbb{R}^{n} e O \in J \subseteq \mathbb{R}$ são conjuntos abertos e $J$ é uma vizinhança de $O \mathrm{em} \mathbb{R}$. Sejam $I=[a, b]$ um intervalo em $\mathbb{R}, U=\{z=x+i y, \Re z \in[a, b],|\Im z|<\delta\}$ e $f \in \mathcal{O}(U)$ uma função holomorfa satisfazendo

$$
|f(z)| \leq \frac{C}{|\Im z|^{N}}, \quad z \in U
$$


como no teorema 1.1. Se $F(x, 0) \in I$ e $\frac{\partial F(x, 0)}{\partial x} \neq 0$ quando $x \in X$, e $F(x, \epsilon) \in U$ quando $x \in X, 0<\epsilon \in J$, segue que

$$
f(F(., \epsilon)) \rightarrow F(., 0)^{*} f(.+i 0) \quad \text { com } \quad \epsilon \rightarrow+0 .
$$

Prova: Como o argumento é local e invariante por mudança de coordenadas, podemos assumir que $F(x, 0)=x_{1}$. Pela prova do teorema 1.1 sabemos que $f=G^{(N+1)}$ onde $G$ é analítica em $\mathbb{Z}$ e contínua em $\overline{\mathbb{Z}}$. Se $\phi \in C_{0}^{\infty}(X)$, por integração por partes com respeito a $x_{1}$ quando $\epsilon \rightarrow 0$, segue que

$$
\begin{aligned}
\int \phi(x) f(F(x, \epsilon)) d x & =\int \phi(x) G^{(N+1)}(F(x, \epsilon)) d x \\
& =\int G(F(x, \epsilon))\left(\partial_{1}\left(-\partial F(x, \epsilon) / \partial x_{1}\right)^{-1}\right)^{N+1} \phi(x) d x \\
& \rightarrow \int G(F(x, 0))\left(-\partial_{1}\right)^{N+1} \phi(x) d x=\int G\left(x_{1}\right)\left(-\partial_{1}\right)^{N+1} \phi(x) d x \\
& =\int<f(.+i 0), \phi\left(., x^{\prime}\right)>d x^{\prime} .
\end{aligned}
$$

Observação: $\mathrm{O}$ conjunto $H$ das matrizes simétricas $A$ com $\Re A$ positiva definida é um conjunto aberto convexo no espaço vetorial complexo $n(n+1) / 2$-dimensional de matrizes simétricas $n \times n$. Se $A \in H$ então $\operatorname{det} A \neq 0$ dado que $A(x)=0, \quad x \in$ $\mathbb{C}^{n}$ implica

$$
0=\Re<A x, \bar{x}>=<(\Re A) x, \bar{x}>,
$$

e daí concluímos que $x=0 .(\operatorname{det} A)^{1 / 2}$ é unicamente definido por continuidade quando $A$ está no fecho de $H$, dado que se $\operatorname{det} A \neq 0$ temos duas ramificações da raiz quadrada em uma vizinhança de $A$, somente uma delas concorda à definição escolhida em $H$. Vamos determinar $(\operatorname{det} A)^{1 / 2}$ quando $A=i B$ é não singular. Podemos assumir que $B$ tem forma diagonal por uma transformação ortogonal real que não muda o $(\operatorname{det} A)^{1 / 2}$ quando $A \in \bar{H}$. Assim, assumimos que $\langle B x, x\rangle=$ $\sum b_{j} x_{j}^{2}, \operatorname{com} b_{j} \neq 0$ para todo $j$. Então

$$
\operatorname{det}(i B+\epsilon I)=\prod\left(\epsilon+i b_{j}\right)
$$

e a raiz quadrada é positiva quando todos os $b_{j}$ identicamente nulos têm o argumento

$$
1 / 2 \sum \arg \left(\epsilon+i b_{j}\right)
$$


onde cada termo está entre $-\pi / 2$ e $\pi / 2$. Portanto,

$$
1 / 2 \sum \arg \left(\epsilon+i b_{j}\right) \rightarrow 1 / 2 \sum \pi / 2 \operatorname{sgn} b_{j} \quad \text { quando } \quad \epsilon \rightarrow 0 \text {. }
$$

Logo

$$
(\operatorname{det}(i B))^{1 / 2}=|\operatorname{det} B|^{1 / 2} e^{(\pi i(\operatorname{sgn} B) / 4)},
$$

onde $\operatorname{sgn} B=\sum \operatorname{sgn} b_{j}$.

Prova do teorema 1.4: Basta verificar só um dos casos, isto é,

$$
P(\partial)(A+i 0)^{(1-n) / 2}=(1-n) \omega_{n+1}|\operatorname{det} A|^{-1 / 2} e^{-\pi i / 2} \delta_{0}
$$

De fato, a conjugação complexa dá o outro caso de (1.7). Primeiro vamos provar que

$$
P(\partial)(A)^{(1-n) / 2}=(1-n) \omega_{n+1}(\operatorname{det} A)^{-1 / 2} \delta_{0},
$$

quando $\Re A$ é positiva definida e $(\operatorname{det} A)^{-1 / 2}$ é definido como em (1.10). De fato $A^{(1-n) / 2}$ é uma extensão homogênea de $A(x)^{(1-n) / 2}$, onde $-\pi / 2<\arg A(x)<\pi / 2$. Pela unicidade da continuidade analítica basta provar (1.12) quando $A$ é positiva definida. Podemos escolher uma bijeção linear $T$ em $\mathbb{R}^{N+1}$ tal que o pullback $T^{*} A$ de $A$ por $T$ é a forma métrica euclidiana. Então

$$
\begin{aligned}
T^{*} P(\partial)(A)^{(1-n) / 2} & =P(\partial) T^{*}(A)^{(1-n) / 2}=P(\partial)|x|^{1-n} \\
& =P(\partial)(1-n) \omega_{n+1} E(x)=(1-n) \omega_{n+1} \delta_{0}
\end{aligned}
$$

e também

$T^{*}\left((1-n) \omega_{n+1}(\operatorname{det} A)^{-1 / 2} \delta_{0}\right)=(1-n) \omega_{n+1}|\operatorname{det} A|^{-1 / 2}|\operatorname{det} T|^{-1} T^{*} \delta_{0}=(1-n) \omega_{n+1} \delta_{0}$.

Logo, pelo teorema 1.3, cada lado de (1.12) tem o mesmo pullback. Provamos (1.12) quando $\Re A$ é positiva-definida. Usamos agora que $A$ é real, não degenerada e sabemos por (1.10) que para $A_{\epsilon}=-i A+\epsilon I, \quad(I=i d), \quad \epsilon>0$ temos que $\left(\operatorname{det} A_{\epsilon}\right)^{-1 / 2} \rightarrow|\operatorname{det} A|^{-1 / 2} e^{i \pi(\operatorname{sgn} A) / 4}, \operatorname{logo}$ aplicando (1.12) para $A_{\epsilon}(x)$ temos

$$
P(\partial) A_{\epsilon}(x)=-i(1-n) \omega_{n+1}|\operatorname{det} A|^{-1 / 2} e^{i \pi(\operatorname{sgn} A) / 4} \delta_{0} .
$$

Mas, pelo Lema 1.3, temos que

$$
A_{\epsilon}^{(1-n) / 2}=\left(e^{-i \pi / 2}(A+\epsilon i I)\right)^{(1-n) / 2} \rightarrow i^{-1} e^{i \pi(n+1) / 4}(A+i 0)^{(1-n) / 2}
$$


em $\mathcal{D}^{\prime}\left(\mathbb{R}^{n} \backslash\{0\}\right)$ e pelo teorema 1.2 , segue que temos convergência em $\mathcal{D}^{\prime}\left(\mathbb{R}^{n}\right)$ para extensões homogêneas. Assim

$$
P(\partial) A_{\epsilon}^{(1-n) / 2}=i^{-1} e^{i \pi(n+1) / 4} P(\partial)(A+i 0)^{(1-n) / 2},
$$

e portanto,

$$
\begin{aligned}
P(\partial)(A+i 0)^{(1-n) / 2} & =e^{-i \pi(n+1) / 4+i \pi(\operatorname{sgn} A) / 4}(1-n) \omega_{n+1}|\operatorname{det} A|^{-1 / 2} \delta_{0} \\
& =(1-n) \omega_{n+1}|\operatorname{det} A|^{-1 / 2} e^{-i \pi / 2} \delta_{0} .
\end{aligned}
$$

Vamos mostrar (1.8). Os dois casos são permutações substituindo $A$ por $-A$. Por (1.5), temos

$$
x_{+}^{a}\left(e^{\pi i a}-e^{-\pi i a}\right)=(x-i 0)^{a} e^{\pi i a}-(x+i 0)^{a} e^{-\pi i a}, \quad \Re a>0 .
$$

Pela observação feita no apêndice $C$ temos que $\Gamma(a+1) \Gamma(-a)=-\pi \sin (\pi a)$ e por (1.6) sabemos que $\chi_{+}^{a}=x_{+}^{a} / \Gamma(a+1)$ então,

$$
\chi_{+}^{a}=i \Gamma(-a) / 2 \pi\left((x-i 0)^{a} e^{\pi i a}-(x+i 0)^{a} e^{-\pi i a}\right), \quad \Re a>0, \quad a \notin \mathbb{Z}_{+},
$$

e, por continuidade analítica, isto é estendido para todo $a \notin \mathbb{Z}_{+}$. Notemos que

$$
(1-n) \omega_{n+1} \Gamma((n-1) / 2) / \pi=-4 \pi^{(n-1) / 2}
$$

e

$$
\pi i / 2+\pi i((1-n) / 2)=\pi i((1-n) / 2)
$$

Vejamos que (1.8) é uma consequência de (1.7). Sabemos que

$$
\begin{aligned}
A^{*} \chi_{+}^{(1-n) / 2} & =\chi_{+}^{(1-n) / 2}(A) \\
& =i \Gamma((n-1) / 2) / 2 \pi\left((A-i 0)^{(1-n) / 2} e^{\pi i(1-n) / 2}-(A+i 0)^{(1-n) / 2} e^{-\pi i(1-n) / 2}\right) .
\end{aligned}
$$

\section{Então}

$$
\begin{aligned}
P(\partial) A^{*} \chi_{+}^{(1-n) / 2} & =i \Gamma((n-1) / 2) / 2 \pi\left[e^{\pi i(1-n) / 2} P(\partial)(A-i 0)^{(1-n) / 2}\right. \\
& \left.-e^{-\pi i(1-n) / 2} P(\partial)(A+i 0)^{(1-n) / 2}\right] \\
& =4 \pi^{(n-1) / 2}|\operatorname{det} A|^{-1 / 2} \delta_{0}\left(e^{\pi i(1-n) / 2} / 2 i-e^{-\pi i(1-n) / 2} / 2 i\right) \\
& =4 \pi^{(n-1) / 2}|\operatorname{det} A|^{-1 / 2} \delta_{0} \sin (\pi n / 2) .
\end{aligned}
$$


Exemplo: [Uma solução fundamental do operador de onda]

O operador de onda em $\mathbb{R}^{n+1}$

$$
\square=\frac{\partial^{2}}{\partial t^{2}}-\Delta
$$

onde $t \in \mathbb{R}$ e $\Delta$ é o laplaciano em $x \in \mathbb{R}^{n}$. Na notação anterior este é o operador $P(\partial)$ se

$$
A=t^{2}-|x|^{2} .
$$

Como um caso particular de (1.8) uma solução fundamental é dada por

$$
E=\frac{\pi^{(1-n) / 2} C A^{*} \chi_{+}^{(1-n) / 2}}{4}, \quad n \geq 1
$$

Isto tem suporte no duplo cone de luz onde $A \geq 0$. 


\section{Capítulo 2}

\section{Cálculo dos operadores Pseudo-diferenciais}

\subsection{Integrais Oscilatórias}

Integrais oscilatórias expressam naturalmente as soluções fundamentais dos operadores de onda. Nosso objetivo é definir rigorosamente expressões do tipo

$$
I_{\phi, a}=\int_{\mathbb{R}^{n}} e^{i \phi(x, \theta)} a(x, \theta) d \theta
$$

para uma certa classe de amplitudes $a(x, \theta) \in C^{\infty}\left(\mathbb{R}^{n}, \mathbb{R}^{n}\right)$. Para este fim, introduzimos as seguintes definições:

Definição 2.1 Sejam $X \subset \mathbb{R}^{n}$ aberto e $\Gamma \subset\left(\mathbb{R}^{N} \backslash\{0\}\right)$ um cone aberto. ${ }^{1}$ Dizemos que uma função $\phi \in C^{\infty}(X \times \Gamma)$ é uma função de fase em $X \times \Gamma$ se e somente se

1. $\phi(x, t \theta)=t \phi(x, \theta)$ se $(x, \theta) \in X \times \Gamma, t>0$

2. $\operatorname{Im} \phi \geq 0$ em $X \times \Gamma$

3. $d \phi \neq 0$ em $X \times \Gamma$.

\footnotetext{
${ }^{1}$ Isto significa que $\Gamma$ é invariante sob multiplicação por escalares positivos da componente em $\mathbb{R}^{N}$.
} 
Definição 2.2 Sejam $m, \rho$, e $\delta$ números reais, com $0<\rho \leq 1$. Denotaremos por $S_{\rho, \delta}^{m}\left(X \times \mathbb{R}^{N}\right)$ o conjunto de todas as funções a $\in C^{\infty}\left(X \times \mathbb{R}^{N}\right)$ tais que para todo compacto $K \subset X$ e todo multi-índice $\alpha, \beta$ vale

$$
\left|D_{x}^{\beta} D_{\theta}^{\alpha} a(x, \theta)\right| \leq C_{\alpha, \beta, K}(1+|\theta|)^{m-\rho|\alpha|+\delta|\beta|},
$$

onde $x \in K, \theta \in \mathbb{R}^{N}$ e $C_{\alpha, \beta, K}$ é constante. Os elementos de $S_{\rho, \delta}^{m}$ são ditos símbolos de ordem m e tipo $\rho, \delta$. Quando $\rho=1$ e $\delta=0$ dizemos que $a \in S_{1,0}^{m}$ é um símbolo clássico e escrevemos simplesmente $a \in S^{m}$

Um exemplo importante de símbolos é aquele descrito pela seguinte proposição.

Proposição 2.1 Seja $m \in \mathbb{N}$ e sejam funções $a_{\alpha} \in C_{b}^{\infty}\left(\mathbb{R}^{n}\right),|\alpha| \leq m$. Então,

$$
p(x, \xi)=\sum_{|\alpha| \leq m} a_{\alpha}(x) \xi^{\alpha}
$$

é um símbolo do tipo (1,0), $p \in S_{1,0}^{m}$, isto é, para todo $\alpha, \beta \in \mathbb{N}^{n} \quad \exists C_{\alpha, \beta}>0$, tal que para todo $x, \xi \in \mathbb{R}^{n}$ vale

$$
\left|\left[D_{x}^{\alpha} D_{\xi}^{\beta} p\right](x, \xi)\right| \leq C_{\alpha, \beta}(1+|| \xi||)^{m-|\beta|}
$$

Prova: Sejam $\beta, \gamma \in \mathbb{N}^{n}$ como todas as derivadas das funções $a_{\alpha}$ são limitadas temos que

$$
\begin{aligned}
\left|\left[D_{x}^{\beta} D_{\xi}^{\gamma} p\right](x, \xi)\right| & =\left|\sum_{|\alpha| \leq m}\left[D_{x}^{\beta} a_{\alpha}\right](x)\left[D_{\xi}^{\gamma} \xi^{\alpha}\right]\right| \\
& \leq \sup _{x \in \mathbb{R}^{n},|\alpha| \leq m}\left|D_{x}^{\beta} a_{\alpha}(x)\right| \sum_{|\alpha| \leq m}\left|D_{\xi}^{\gamma} \xi^{\alpha}\right| \\
& \leq C_{1}(\beta) \sum_{|\alpha| \leq m}\left|D_{\xi}^{\gamma} \xi^{\alpha}\right| .
\end{aligned}
$$


Além disso,

$$
\begin{aligned}
\left|D^{\gamma} \xi^{\alpha}\right| & =\left|\gamma !\left(\begin{array}{c}
\alpha \\
\gamma
\end{array}\right) \xi^{\alpha-\gamma}\right| \\
& \leq \gamma !\left(\begin{array}{c}
\alpha \\
\gamma
\end{array}\right)\left(1+\|\xi\|^{2}\right)^{|\alpha|-|\gamma|} \\
& \leq \gamma !\left(\begin{array}{c}
\alpha \\
\gamma
\end{array}\right)\left(1+\|\xi\|^{2}\right)^{m-|\gamma|} \\
& \leq C\left(1+\|\xi\|^{2}\right)^{m-|\gamma|}
\end{aligned}
$$

onde $C=\max \left\{\gamma !\left(\begin{array}{c}\alpha \\ \gamma\end{array}\right) ;|\alpha| \leq m, 0<|\gamma| \leq|\alpha|\right\}$, para todo $\gamma \in \mathbb{N}^{n},|\alpha| \leq m$.

Da desigualdade

$$
(1+|t|)^{2}=1+2|t|+|t|^{2} \leq 1+\left(1+|t|^{2}\right)+|t|^{2}=2\left(1+|t|^{2}\right) \leq 2(1+|t|)^{2}
$$

temos

$$
1 / 2(1+\|\xi\|) \leq\left(1+\|\xi\|^{2}\right)^{1 / 2} \leq 1+\|\xi\| \text { para todo } \xi \in \mathbb{R}^{n} .
$$

Então

$$
\left|\left[D_{x}^{\beta} D_{\xi}^{\gamma} p\right](x, \xi)\right| \leq C_{1}(\beta) C(1+|| \xi||)^{m-|\gamma|} .
$$

Para o estudo das integrais oscilatórias vamos analisar os funcionais lineares $I_{\phi, a}: C_{c}^{\infty} \mapsto \mathbb{R}$ definidos por:

$$
I_{\phi, a}(u)=\iint e^{i \phi(x, \theta)} a(x, \theta) u(x) d \theta d x .
$$

O seguinte teorema fundamental permite que interpretemos uma integral oscilatória como uma distribuição.

\section{Teorema 2.1 (Integral oscilatória)}

Sejam $\Gamma \subset X \times \mathbb{R}^{N}$ um cone aberto, $F \subset \Gamma \cup(X \times\{0\})$ um cone fechado, e $\phi \in \Gamma$ uma função fase em $\Gamma$. Então o funcional

$$
I_{\phi, a}: u \mapsto I_{\phi, a}(u)
$$


definido por

$$
I_{\phi, a}(u)=\iint e^{i \phi(x, \theta)} a(x, \theta) u(x) d x d \theta
$$

quando a integral é absolutamente convergente pode ser extendida a um único caminho para todo $a \in \cup S_{\rho, \delta}^{m}\left(X \times \mathbb{R}^{N}\right)$ com $m \in \mathbb{Z}^{+}, \rho \in(0,1], \delta \in[0,1)$ com suporte em $F$ e todo $u \in C_{0}^{\infty}(X)$, tal que $I_{\phi, a}(u)$ é uma função linear continua. A forma linear

$$
I_{\phi, a}: u \mapsto I_{\phi, a}(u)
$$

define uma distribuição de ordem $\leq k$ caso tenhamos

$$
m-k \rho<-N, \quad m-k(1-\delta)<-N .
$$

Para provar este teorema precisamos do seguinte teorema:

Teorema 2.2 Sejam $K \subset \mathbb{R}^{n}$ um conjunto compacto, $X$ uma vizinhança aberta de $K$ e $k$ inteiro não negativo. Se $u \in C_{0}^{k}(K), \quad e \quad f \in C^{k+1}(X)$ é real, então

$$
\left|\int u(x) e^{i \lambda f(x)} d x\right| \leq \frac{c}{\lambda^{k}} \sum_{|\alpha| \leq k} \sup \left|D^{\alpha} u\right|\left|f^{\prime}\right|^{|\alpha|-2 k}, \quad \lambda>0 .
$$

Prova: Vamos fazer a prova por indução. Quando $k=0$ temos

$$
\left|\int u(x) e^{i \lambda f(x)} d x\right| \leq C \sup (u) \text {. }
$$

Por isso podemos assumir na prova que $k>0$ e que (2.4) é provado para valores menores que $k$. Definamos $N=\left|f^{\prime}\right|^{2}$.

Observemos que

$$
N u=u \sum_{1}^{n}\left|\partial f / \partial x_{\nu}\right|^{2}
$$

Assim, $u=\sum_{1}^{n} u_{\nu} \partial f / \partial x_{\nu}$ se $N u_{\nu}=u \partial \bar{f} / \partial x_{\nu}$ quando $\nu \neq 0$.

Dado que $i \lambda \partial f / \partial x_{\nu} e^{i \lambda f}=\partial_{\nu} e^{i \lambda f}$ obtemos, integrando por partes,

$$
\begin{aligned}
\int u(x) e^{i \lambda f(x)} d x & =\int \sum_{1}^{n} u_{\nu} \partial f / \partial x_{\nu} e^{i \lambda f(x)} d x \\
& =\sum_{1}^{n} \int \frac{1}{i \lambda} u_{\nu} \partial_{\nu} e^{i \lambda f} d x \\
& =\sum_{1}^{n} \frac{1}{i \lambda} \int e^{i \lambda f} \partial_{\nu} u_{\nu} d x
\end{aligned}
$$


Pela hipótese de indução e com a notação

$$
|u|_{\mu}=\sum_{|\alpha|=\mu}\left|\partial^{\alpha} u\right|
$$

temos que

$$
\begin{aligned}
\lambda^{k}\left|\int u(x) e^{i \lambda f(x)} d x\right| & =\lambda^{k}\left|\sum_{1}^{n} \frac{1}{i \lambda} \int e^{i \lambda f(x)} \partial_{\nu} u_{\nu} d x\right| \\
& =\left|\sum_{1}^{n} \frac{\lambda^{k-1}}{i} \int e^{i \lambda f} \partial_{\nu} u_{\nu} d x\right| \\
& \leq 1 / i \sum_{1}^{n}\left|\lambda^{k-1} \int e^{i \lambda f} \partial_{\nu} u_{\nu} d x\right| \\
& \leq\left.\frac{1}{i} C \sum_{\nu=1}^{n} \sum_{|\alpha| \leq k-1} \sup \left|D^{\alpha}\left(\partial_{\nu} u_{\nu}\right)\right| f\right|^{|\alpha|-2(k-1)} \\
& \leq C \sup \sum_{\nu=1}^{n} \sum_{\mu=0}^{k-1} \sum_{|\alpha|=\mu} \partial^{\alpha}\left(\partial_{\nu} u_{\nu}\right) N^{|\alpha| / 2-k+1} \\
& =C \sup \sum_{\mu=0}^{k-1} \sum_{\nu=1}^{n}\left|u_{\nu}\right|_{\mu+1} N^{|\mu| / 2-k+1}
\end{aligned}
$$

ou seja,

$$
\lambda^{k}\left|\int u(x) e^{i \lambda f(x)} d x\right| \leq C \sup \sum_{\mu=0}^{k-1} \sum_{\nu=1}^{n}\left|u_{\nu}\right|_{\mu+1} N^{|\mu| / 2-k+1} .
$$

Para estimar o lado direito precisamos do seguinte lema:

Lema 2.1 Se $g \in C^{2}(-\delta, \delta)$ é não negativa, então

$$
\left|g^{\prime}(0)\right|^{2} \leq \frac{g(0)}{\delta^{2}}\left(g(0)+2 \sup _{|x| \leq \delta} \delta^{2}\left|g^{\prime \prime}(x)\right|\right) .
$$

Prova: A prova é reduzida para o caso $\delta=1$ se $x / \delta$ é uma nova variável. Podemos assumir que $g(0)=1$.

Pela fórmula de Taylor temos

$$
1+g^{\prime}(x)+M x^{2} / 2 \geq g(x) \geq 0, \quad|x|<1 ; \quad M=\sup \left|g^{\prime \prime}\right| .
$$

Se $M \leq 2$ tomamos $x^{2}=1$ e temos para $x=1 \quad g^{\prime}(0) \geq-1-M / 2$ e para $x=-1 \quad g^{\prime}(0) \geq 1+M / 2$ portanto $\left|g^{\prime}(0)\right| \geq 1+M / 2 \leq(1+2 M)^{1 / 2}$ dado que $M^{2} / 4 \leq M$, e se $M>2$ tomamos $x^{2}=2 / M$ então $\left|g^{\prime}(0)\right| \geq 2 /|x| \leq(2 M)^{1 / 2}$. 
Fim da prova do teorema 2.2: Aplicando o lema 2.1 a $f$ temos

$$
\left|\partial_{\nu} f\right|^{2} \leq C f
$$

Basta provar que

$$
N^{1 / 2} \sum_{\nu=1}^{n}\left|u_{\nu}\right|_{\mu} \leq C \sum_{r=0}^{\mu}|u|_{r}|N|^{(r-\mu) / 2} .
$$

Por indução, temos que para $\mu=0$ pela definição de $N$ e $u_{\nu}$ temos

$$
\begin{aligned}
N^{1 / 2} \sum_{\nu=1}^{n} u_{\nu} & =N^{1 / 2} \sum_{\nu=1}^{n} \frac{u}{N} \partial \bar{f} / \partial x_{\nu} \\
& \leq u C\left(\sum_{\nu=1}^{n} \frac{\left(\partial f / \partial x_{\nu}\right)^{2}}{N}\right)^{1 / 2}=C u .
\end{aligned}
$$

Podemos assumir que $0<\mu \leq k$ e que a estimativa é provada para valores menores que $\mu$. Seja $|\alpha|=\mu$ e aplicamos $\partial^{\alpha}$ para $N u_{\nu}=u \partial \bar{f} / \partial x_{\nu}$ quando $\nu \neq 0$. Pela desigualdade triangular, e como $f \in C^{k+1}(X)$ com $X$ sendo uma vizinhança do compacto $K$, então $\partial^{\alpha-\beta} N$ e $\partial^{\alpha-\beta}\left(\partial \bar{f} / \partial x_{\nu}\right)$ são limitadas para $\beta>1$ e assim

$$
N\left|u_{\nu}\right|_{\mu} \leq C\left(|N|_{1}\left|u_{\nu}\right|_{\mu-1}+\left|u_{\nu}\right|_{\mu-2}+\ldots+\left|u_{\nu}\right|_{0}+|u|_{\mu}|f|_{1}+|u|_{\mu-1}+\ldots+|u|_{0}\right),
$$

pelo lema 2.1 e pela hipótese de indução temos

$$
\begin{aligned}
N\left|u_{\nu}\right|_{\mu} & \leq C^{\prime}\left(N^{1 / 2}\left|u_{\nu}\right|_{\mu-1}+\left|u_{\nu}\right|_{\mu-2}+\ldots+\left|u_{\nu}\right|_{0}+|u|_{\mu} N^{1 / 2}+|u|_{\mu-1}+\ldots+|u|_{0}\right) \\
& \leq C^{\prime \prime} \sum_{r=0}^{\mu}|u|_{r}|N|^{(r-\mu+1) / 2} .
\end{aligned}
$$

Portanto, substituindo (2.7) em (2.5) obtemos (2.4).

Prova do teorema 2.1: Considere uma partição diádica da unidade, isto é $\left\{\chi_{\nu}\right\}$ tal que $\sum_{\nu=0}^{\infty} \chi_{\nu}=1$ e $2^{\nu-1} \leq|\theta| \leq 2^{\nu+1}$ quando $\theta \in \operatorname{supp} \chi_{\nu}, \nu \neq 0$. Isto pode ser conseguido tomando $\chi \in C_{0}^{\infty}\left(\mathbb{R}^{N}\right)$, com $\chi(\theta)=1$ quando $|\theta|<1$ e $\chi(\theta)=0$ quando $|\theta|>2$, já que

$$
\chi_{\nu}(\theta)=\chi\left(2^{-\nu} \theta\right)-\chi\left(2^{1-\nu} \theta\right), \quad \nu>0 ; \quad \chi_{0}(\theta)=\chi(\theta) .
$$


Se $a \in S_{\rho, \delta}^{m}$ e $x \in K \Subset X$, temos

$$
\left|D_{x}^{\beta} D_{\theta}^{\alpha}\left(\chi_{\nu}(\theta)-1\right) a(x, \theta)\right| \leq C_{\alpha, \beta, K}(1+|\theta|)^{m^{\prime}-\rho|\alpha|+\delta|\beta|},
$$

visto que $\left|D^{\alpha} \chi_{\nu}(\theta)\right| \leq C_{\alpha}(1+|\theta|)^{-|\alpha|}$, com $C_{\alpha}$ uma constante independente de $\nu$. Daí a série $\sum_{\nu=0}^{\infty} \chi_{\nu} a$ converge para $a$ em $S_{\rho, \delta}^{m^{\prime}}$ se $m^{\prime}>m$, já que no máximo dois termos na série têm suportes coincidindo. Se existe uma extensão de $I_{\phi}$ com as propriedades requeridas, ela é dada por

$$
I_{\phi, a}(u)=\sum_{\nu} I_{\phi, \chi_{\nu} a}(u)
$$

O teorema fica provado se mostrarmos que a série do lado direito converge e que as somas têm as propriedades do teorema.

Para $\nu \geq 0$ e

$$
\begin{aligned}
\chi_{\nu+1}(\theta) & =\chi\left(2^{-\nu-1} \theta\right)-\chi\left(2^{-\nu} \theta\right)=\chi_{1}\left(2^{-\nu} \theta\right) I_{\phi}\left(\chi_{\nu+1} a u\right) \\
& =\int e^{i \phi(x, \theta)} \chi\left(2^{-\nu} \theta\right) a(x, \theta) u(x) d x d \theta \\
& =2^{N \nu} \int e^{i \omega \phi(x, \theta)} \chi_{1}(\theta) a(x, \omega \theta) u(x) d x d \theta
\end{aligned}
$$

$\operatorname{com} \omega=2^{\nu}$.

Por hipótese $\gamma=\max (\delta, 1-\rho)<1$, e se

$$
\left|D_{x}^{\beta} D_{\theta}^{\alpha} a(x, \theta)\right| \leq M(1+|\theta|)^{m-\rho|\alpha|+\delta|\beta|}, \quad|\alpha+\beta| \leq k, \quad x \in K,
$$

temos

$$
\left|D_{x}^{\alpha} D_{\theta}^{\alpha} a\left(x, 2^{\nu} \theta\right)\right| \leq C M 2^{\nu(m+\gamma|\rho|)}
$$

se $x \in K, \quad 1 / 2<|\theta|<2, \quad|\alpha| \leq k$ do teorema 2.2 segue que

$$
\left|I_{\phi}\left(\chi_{\nu+1} a u\right)\right| \leq C M 2^{\nu(N+m+\gamma k-k)} \sum_{|\alpha| \leq k} \sup \left|D^{\alpha} u\right|, \quad u \in C_{0}^{\infty}(K) .
$$

Quando $(1-\gamma) k>N+m$, concluímos que (2.8) converge e que $u \mapsto I_{\phi, a}(u)$ é uma distribuição de ordem $\leq k$. 
Definição 2.3 A definição extendida de (2.3), na qual $a(x, \theta) \in S_{\rho, \delta}^{m}$ e $\phi(x, \theta)$ é uma função fase, é dita ser uma integral oscilatória. Para a distribuição $u \mapsto I_{\phi, a}(u)$ será usada a notação

$$
I_{\phi, a}=\int_{\mathbb{R}^{n}} e^{i \phi(x, \theta)} a(x, \theta) d \theta .
$$

Exemplo: (Transformada inversa de Fourier)

$$
\int_{\mathbb{R}^{n}} e^{i x \cdot \theta} d \theta=\delta_{0}(x)(2 \pi)^{n}
$$

Por definição consideramos a integral oscilatória

$$
\iint e^{i x \cdot \theta} u(x) d x d \theta, \quad u \in C_{0}^{\infty}\left(\mathbb{R}^{n}\right) .
$$

Se $\chi \in C_{0}^{\infty}$ é 1 numa vizinhança de zero, temos $\chi(. / t) \rightarrow 1$ em $S_{1,0}^{m}$ para qualquer $m>0$ com $t \rightarrow \infty$. Assim a integral oscilatória é o limite da integral dupla convergente

$$
\begin{aligned}
& \iint e^{i x \cdot \theta} \chi(\theta / t) u(x) d x d \theta=\iint e^{-i \theta / t(-t x)} \chi(\theta / t) u(x) d x d \theta=\int t^{n} \widehat{\chi}(-t x) u(x) d x \\
& =\int \widehat{\chi}(-x) u(x / t) d x \rightarrow u(0) \int \widehat{\chi}(-x) d x=u(0)(2 \pi)^{n} \chi(0)=u(0)(2 \pi)^{n},
\end{aligned}
$$

e isto é uma outra maneira para expressar a fórmula inversa de Fourier.

Nosso objetivo é descrever as irregularidades da distribuição $I_{\phi, a}$. Vamos ver que os pontos singulares estão contidos no conjunto de pontos críticos da função de fase.

Teorema 2.3 Para a distribuição $I_{\phi, a}$ definida por (2.3) temos

$$
\operatorname{suppsing}\left(I_{\phi, a}\right) \subset \operatorname{Crit}(\phi)=\left\{x \in X ; \phi_{\theta}^{\prime}(x, \theta)=0 \quad \text { para algum }(x, \theta) \in F\right\} .
$$

$A$ restrição de $I_{\phi, a}$ para $X \backslash C r i t(\phi)$ é portanto uma função $C^{\infty}$

$$
x \mapsto \int e^{i \phi(x, \theta)} a(x, \theta) d \theta,
$$

que é definida para $x$ fixo como uma integral oscilatória. 
Prova: A definição de $\operatorname{Crit}(\phi)$ significa que $\phi(x, \theta)$ é uma função de fase de $\theta$ quando $x$ é fixo em $X \backslash C$ rit $(\phi)$ então a integral oscilatória em (2.9) é definida. Esta é uma função contínua de $x$, para provar a existência da integral oscilatória provamos que é o limite localmente uniforme com respeito a $x$, da função $C^{\infty}$

$$
\int e^{i \phi(x, \theta)} a(x, \theta) \chi(\theta / t) d \theta
$$

A derivada com respeito a $x$ é

$$
\int e^{i \phi(x, \theta)}\left(i \phi_{x}^{\prime}(x, \theta) a(x, \theta)+a_{x}^{\prime}(x, \theta)\right) \chi(\theta / t) d \theta,
$$

a qual converge para a integral oscilatória obtida diferenciando (2.9) sob o sinal de integração. Assim a função (2.9) pertence a $C^{1}(X \backslash$ Crit $(\phi))$ e a derivada pode ser computada por diferenciação formal sob o sinal de integração. Visto que $\phi_{x}^{\prime}(x, \theta) a(x, \theta)(1-\chi(\theta)) \in S_{\rho, \delta}^{m+1}$, podemos repetir o processo e concluir que (2.9) é uma função $C^{\infty}(X \backslash C r i t(\phi))$. Esta função é igual à distribuição $u \mapsto I_{\phi}(a u)$, se $u \in C_{0}^{\infty}(X \backslash \operatorname{Crit}(\phi))$ temos

$$
\begin{aligned}
I_{\phi}(a u) & =\lim _{t \rightarrow \infty} \int u(x) d x\left(\int e^{i \phi(x, \theta)} a(x, \theta) \chi(\theta / t) d \theta\right) \\
& =\int u(x) d x\left(\int e^{i \phi(x, \theta)} a(x, \theta) d \theta\right) .
\end{aligned}
$$

\subsection{Estimativas da fase estacionária:}

O método da fase estacionária é de central importância em análise clássica dado que integrais da forma

$$
I(\lambda)=\int_{R^{N}} e^{i \lambda \Phi(y)} a(y) d y, \quad \lambda>0
$$

surgem naturalmente como soluções fundamentais de certos operadores diferenciais em muitos problemas. Vamos estudar estas integrais quando $\Phi \in C^{\infty}$ é real e $a \in C_{0}^{\infty}$. Se $\Phi$ é uma constante, o fator $e^{i \lambda \Phi(y)}$ torna-se altamente oscilatório quando $\lambda \rightarrow \infty$ e, por causa disto, esperamos que $I(\lambda)$ decaia quando $\lambda$ é grande. A fase estacionária é uma ferramenta que permite fazer estimativas precisas para essas integrais quando a função fase $\Phi$ satisfaz condições naturais envolvendo suas derivadas. 


\section{Lema 2.2 (Fase não-estacionária)}

Sejam $a \in C_{0}^{\infty} e \Phi \in C^{\infty}$ tais que $\left|\nabla_{x} \Phi\right| \geq c>0$ sob supp a. Então, para $\lambda>1$,

$$
\left|\int e^{i \lambda \Phi(x)} a(x) d x\right| \leq C_{N} \lambda^{-N}, \quad N=1,2 \ldots
$$

onde $C_{N}$ depende somente de c se $\Phi$ e a pertencem a um subconjunto limitado de $C^{\infty}$ e a é suportada num conjunto compacto fixo.

Prova: Dado $x_{0} \in \operatorname{supp} a$ existe uma direção $\nu$ tal que $|\langle\nu, \nabla \Phi\rangle| \geq c / 2$ sob alguma bola centrada em $x_{0}$. Assim, por compacidade, podemos escolher uma partição da unidade finita $\alpha_{j} \in C_{0}^{\infty}$ a que correspondem vetores unitários $\nu_{j}$ tal que $\sum_{\text {suppa }} \alpha_{j}(x)=1$ e $|\langle\nu, \nabla \Phi\rangle| \geq c / 2$ sob supp $\alpha_{j}$. Se colocarmos $a_{j}(x)=\alpha_{j}(x) a(x)$, é suficiente provar que para cada $j$

$$
\left|\int e^{i \lambda \Phi(x)} a_{j}(x) d x\right| \leq C_{N} \lambda^{-N} .
$$

Através de uma mudança de coordenadas podemos assumir que $\nu_{j}=(1,0, \ldots 0)$, o que significa que $\left|\frac{\partial \Phi}{\partial x_{1}}\right| \geq c / 2$ sob supp $\alpha_{j}$. Se definimos

$$
L(x, D)=\frac{1}{i \lambda \frac{\partial \Phi}{\partial x_{1}}} \frac{\partial}{\partial x_{1}},
$$

então $L(x, D) e^{i \lambda \Phi(x)}=e^{i \lambda \Phi(x)}$. Como consequência temos que se $L^{*}=L^{*}(x, \dot{D})=$ $\frac{\partial}{\partial x_{1}}\left(\frac{1}{i \lambda \frac{\partial \Phi}{\partial x_{1}}}\right)$ é o adjunto, então

$$
\int e^{i \lambda \Phi(x)} a_{j}(x) d x=\int e^{i \lambda \Phi(x)}\left(L^{*}\right)^{N} a_{j}(x) d x .
$$

As suposições implicam que

$$
\left(L^{*}\right)^{N} a_{j}=O\left(\lambda^{-N}\right) .
$$

Este lema diz que

$$
I(\lambda)=O\left(\lambda^{-N}\right), \quad \forall N \quad \text { se } \quad \nabla \Phi \neq 0 .
$$

Se $\nabla \Phi=0$ em algum lugar, mas o determinante da matriz $n \times n \quad\left(\frac{\partial^{2} \Phi}{\partial y_{j} \partial y_{k}}\right)$ não é identicamente nulo, então

$$
I(\lambda)=O\left(\lambda^{-n / 2}\right)
$$


Vamos considerar integrais oscilatórias 1-dimensional que envolvem funções fase não degeneradas com pontos críticos não degenerados e as amplitudes envolvem o parâmetro $\lambda$. A condição natural para $a(\lambda, y)$ é que são identicamente nulas quando não pertencem a um conjunto compacto fixo, e que

$$
\left|\left(\frac{\partial}{\partial y}\right)^{\gamma}\left(\frac{\partial}{\partial \lambda}\right)^{\alpha} a(\lambda, y)\right| \leq C_{\alpha \gamma}(1+\lambda)^{-\alpha}
$$

para todo $\alpha, \gamma$. Para melhor entendermos esta situação vamos considerar inicialmente o caso 1-dimensional para depois analisarmos o caso n-dimensional.

\subsubsection{Caso 1-dimensional:}

Teorema 2.4 Com as mesmas condições anteriores e supondo que $\Phi(0), \Phi^{\prime}(0)=$ 0 e $\Phi^{\prime}(y) \neq 0$ sob supp $a(\lambda,.) \backslash\{0\}$. Seja

$$
I(\lambda)=\int e^{i \lambda \Phi(y)} a(\lambda, y) d y
$$

então se $\Phi^{\prime \prime}(0) \neq 0$ e $\alpha=0,1,2, \ldots$,

$$
\left|\left(\frac{\partial}{\partial \lambda}\right)^{\alpha} I(\lambda)\right| \leq C_{\alpha}(1+\lambda)^{-1 / 2-\alpha} .
$$

Prova: Para provar (2.12) notemos que, pela regra do produto,

$$
\left(\frac{\partial}{\partial \lambda}\right)^{\alpha} I(\lambda)=\sum_{j+k=\alpha} \frac{\alpha !}{j ! k !} \int_{0}^{\infty} e^{i \lambda \Phi(y)}(i \Phi(y))^{j}\left(\frac{\partial}{\partial \lambda}\right)^{k} a(\lambda, y) d y .
$$

Mas, as hipóteses sob $\Phi$ e o teorema de Taylor implicam que existe uma função $\eta$ não nula tal que

$$
\Phi(y)=y^{2} \eta(y) \quad \text { sob supp } \quad a .
$$

Daí,

$$
\Phi^{\alpha}=y^{2 \alpha} \eta^{\alpha} .
$$

Assim, por (2.11), vemos que (2.12) segue do seguinte lema:

\section{Lema 2.3 (Van der Corput)}

Seja $\Phi$ tal que $\Phi(0), \Phi^{\prime}(0)=0$ e $\Phi^{\prime}(y) \neq 0$ sob supp $a(\lambda,.) \backslash\{0\}$ como no teorema 2.4. Então, para $k=0,1,2, \ldots$,

$$
\left|\int_{0}^{\infty} e^{i \lambda \Phi(y)}(y)^{k} a(\lambda, y) d y\right| \leq C_{k} \lambda^{-1 / 2-k / 2} .
$$


Prova: Denotemos por $I_{k}$ a integral em (2.13). Para estimar isto fixamos uma função $\rho(y) \in C^{\infty}$ que é igual a 1 quando $y<1$ e zero para $y>2$. Então, para $\delta>0$,

$$
I_{k}=\int_{0}^{\infty} e^{i \lambda \Phi}(y)^{k} a(\lambda, y) \rho(y / \delta) d y+\int_{0}^{\infty} e^{i \lambda \Phi}(y)^{k} a(\lambda, y)(1-\rho(y / \delta)) d y=I+I I .
$$

Temos

$$
|I| \leq C \int_{0}^{2 \delta}(y)^{k} d y=C^{\prime} \delta^{1+k}
$$

para a segunda integral vamos fazer integração por partes. Seja

$$
L^{*}(x, D)=\frac{\partial}{\partial y} \frac{1}{i \lambda \Phi^{\prime}} .
$$

Então para $N=0,1, \ldots$

$$
\begin{aligned}
|I I| & =\left|\int e^{i \lambda \Phi}\left(L^{*}(x, D)\right)^{N}\left(y^{k} a(\lambda, y)(1-\rho(y / \delta))\right) d y\right| \\
& \leq \int_{y>\delta}\left|\left(L^{*}(x, D)\right)^{N}\left(y^{k} a(\lambda, y)(1-\rho(y / \delta))\right)\right| d y .
\end{aligned}
$$

De qualquer maneira

$$
\left|\frac{1}{\Phi^{\prime}(y)}\right| \leq C \frac{1}{y}
$$

e também a regra do produto para diferenciação implica que o último integrando em (2.14) é majorado por

$$
\lambda^{-N} \max \left(y^{k-2 N}, y^{k-N} \delta^{-N}\right) \quad \text { para qualquer } \quad N .
$$

Portanto se o suporte de $a$ está contido em $[-C, C]$, onde $C<\infty$, isto significa que, para $N \geq k+2$, podemos dominar $|I I|$ por

$$
\lambda^{-N} \int_{\delta}^{C}\left(y^{k-2 N}+y^{k-N} \delta^{-N}\right) d y \leq \lambda^{-N} \delta^{1+k-2 N} .
$$

Junto com nossas estimativas, concluímos que

$$
\left|I_{k}\right| \leq C\left(\delta^{1+k}+\lambda^{-N} \delta^{1+k-2 N}\right) .
$$

O lado direito é menor quando os dois somandos concordam, isto é, quando $\delta=\lambda^{-1 / 2}$, o que dá

$$
\left|I_{k}\right| \leq C \lambda^{-1 / 2-k / 2} .
$$


Exemplo: (Funções de Bessel)

$$
J_{k}(\lambda)=(2 \pi)^{-1} \int_{0}^{2 \pi} e^{i \lambda \operatorname{sen} \theta} e^{i k \theta} d \theta, \quad k=0,1, \ldots
$$

O teorema 2.4 implica que

$$
\left|J_{k}(\lambda)\right| \leq C_{k} \lambda^{-1 / 2}
$$

Para muitos problemas é útil ter uma versão com coeficientes variáveis do teorema 2.4. Provaremos o seguinte resultado:

Corolário 2.1 Suponhamos que $\Phi(x, y) \in C^{\infty}\left(\mathbb{R}^{n} \times \mathbb{R}^{n}\right)$ uma função de fase real tal que

$$
\Phi_{y}^{\prime}(0,0)=0, \quad \Phi_{y y}^{\prime \prime}(0,0) \neq 0
$$

e seja $a(\lambda, x, y) \in C^{\infty}\left(\mathbb{R} \times \mathbb{R}^{n} \times \mathbb{R}^{n}\right)$ uma função que tem o y-suporte suficientemente pequeno, satisfazendo:

$$
\left|\left(\frac{\partial}{\partial \lambda}\right)^{\alpha}\left(\frac{\partial}{\partial x}\right)^{\beta_{1}}\left(\frac{\partial}{\partial y}\right)^{\beta_{2}} a(\lambda, x, y)\right| \leq C_{\alpha \beta}(1+\lambda)^{-\alpha} .
$$

Então, para $I(x, \lambda)=\int e^{i \lambda \Phi(x, y)} a(\lambda, x, y) d y$ e $\alpha, \beta=0,1,2, \ldots$, temos

$$
\left|\left(\frac{\partial}{\partial \lambda}\right)^{\alpha}\left(\frac{\partial}{\partial x}\right)^{\beta}\left(e^{-i \lambda \Phi(x, y(x))} I(x, \lambda)\right)\right| \leq C_{\alpha \beta}(1+\lambda)^{-1 / 2-\alpha}
$$

quando $x$ é pequeno.

Prova: Pelo teorema da função implícita, pode existir uma função $y(x) \in C^{\infty}$ para a equação

$$
\Phi_{y}^{\prime}(x, y(x))=0
$$

com $y(0)=0$, quando $x$ é suficientemente pequeno. Colocamos

$$
\widetilde{\Phi}(x, y)=\Phi(x, y)=\Phi(x, y(x)) \text {, }
$$

e notemos que esta função fase é igual a zero quando $y=y(x)$. Então, se $\beta=0$, a quantidade necessária para a estimativa é

$$
\sum_{j+k=\alpha} \frac{\alpha !}{j ! k !}\left(\frac{\partial}{\partial \lambda}\right)^{j}\left(e^{i \lambda \tilde{\Phi}(x, y(x))}\right)\left(\frac{\partial}{\partial \lambda}\right)^{k} a(\lambda, x, y) d y .
$$


De qualquer maneira, pelo teorema 2.4 e por (2.16), $\alpha$ vezes tal somando é $O\left(\lambda^{-1 / 2-\alpha}\right)$, o que implica (2.17) para este caso especial. Para tratar o caso envolvendo $\beta \neq 0$, note que $\widetilde{\Phi}$ tem um zero de ordem 2 na variável $y$ quando $y=y(x)$. Por isto, vemos que o argumento acima prova que a desigualdade para $\beta$ arbitrário segue de (2.13).

Observação: Se $\Phi^{(j)}(0)=0$ para $0 \leq j \leq m-1$ mas $\Phi^{(m)}(0) \neq 0$ então

$$
\left|\int_{0}^{\infty} e^{i \lambda \Phi(y)} a(y) d y\right| \leq C \lambda^{-1 / m}
$$

desde que $a$ tem suporte suficientemente pequeno.

\subsubsection{Fase estacionária em dimensões superiores:}

Seja a integral oscilatória n-dimensional

$$
I(\lambda)=\int_{\mathbb{R}^{n}} e^{i \lambda \Phi(y)} a(\lambda, y) d y, \quad \lambda>0
$$

com funções $\Phi, a \in C^{\infty}$, com $\Phi$ real e $a$ com suporte compacto. Vamos estender o teorema 2.4. A função fase $\Phi$ tem pontos críticos não degenerados, notemos que pontos críticos não degenerados podem ser isolados dado que, pelo teorema de Taylor, se chamamos $H=\left(\frac{\partial^{2} \Phi}{\partial y_{j} \partial y_{k}}\right)$ a matriz Hessiana então próximo do ponto crítico $y_{0}$,

$$
\Phi(y)=1 / 2<H\left(y-y_{0}\right),\left(y-y_{0}\right)>+O\left(\left|y-y_{0}\right|^{3}\right),
$$

e daí

$$
\nabla \Phi(y)=H\left(y-y_{0}\right)+O\left(\left|y-y_{0}\right|^{2}\right) .
$$

Finalmente, dizemos que $\Phi$ é uma função fase não degenerada se todos seus pontos são não degenerados. As amplitudes têm as mesmas propriedades anteriores.

Teorema 2.5 Suponhamos que a é como acima, $\Phi(0)=0$ e 0 é um ponto crítico não degenerado de $\Phi$. Seja

$$
I(\lambda)=\int_{\mathbb{R}^{n}} e^{i \lambda \Phi(y)} a(\lambda, y) d y .
$$


Então, se $\nabla \Phi(y) \neq 0$ sob supp $a(\lambda,.) \backslash\{0\}$,

$$
\left|\left(\frac{\partial}{\partial \lambda}\right)^{\alpha} I(\lambda)\right| \leq C_{\alpha}(1+\lambda)^{-n / 2-\alpha} .
$$

Primeiro vamos provar este teorema para o caso quando

$$
\Phi=Q(y)=1 / 2\left(y_{1}^{2}+\ldots+y_{j}^{2}-y_{j+1}^{2} \ldots-y_{n}^{2}\right) .
$$

A forma quadrática $Q$ é não degenerada dado que a matriz Hessiana é a matriz diagonal de 1 e -1 .

Teorema 2.6 Se $Q$ e a são como acima,

$$
\left(\frac{\partial}{\partial \lambda}\right)^{\alpha} \int e^{i \lambda Q(y)} a(\lambda, y) d y=O\left(\lambda^{-n / 2-|\alpha|}\right) .
$$

A prova deste teorema é uma consequência do seguinte lema.

Lema 2.4 Se, para um multi-índice $\alpha$ dado, temos $y^{\alpha}=y_{1}^{\alpha_{1}} \ldots y_{n}^{\alpha_{n}}$, então

$$
\left|\int e^{i \lambda Q(y)} a(\lambda, y) y^{\alpha} d y\right| \leq(1+\lambda)^{-[n+|\alpha|] / 2} .
$$

Além disso, se $|\alpha|$ é impar, a integral é

$$
O\left((1+\lambda)^{-[n+|\alpha|+1] / 2}\right) .
$$

Prova: Por indução. No lema anterior vimos que (2.24) mantém-se para $n=1$. Por isso, assumimos que o resultado é certo para $n-1$. Escrevamos a integral em (2.24) como

$$
\int_{R^{n-1}} e^{i \lambda Q\left(y^{\prime}\right)}\left\{\int_{-\infty}^{\infty} e^{i \lambda y_{1}^{2} / 2} a\left(\lambda, y_{1}, y^{\prime}\right) y_{1}^{\alpha_{1}} d y_{1}\right\}\left(y^{\prime}\right)^{\alpha^{\prime}} d y^{\prime}
$$

onde $Q\left(y^{\prime}\right)=Q(y)+y_{1}^{2} / 2$ (de fato, é uma função não degenerada). Pelo Lema 2.3 de (Van der Corput), podemos controlar a integral interna, de fato (2.13) implica que a integral interna é igual a uma função $\widetilde{a}\left(\lambda, y^{\prime}\right)$ a qual tem suporte compacto na variável $y^{\prime}$ e satisfaz

$$
\left|\left(\frac{\partial}{\partial \lambda}\right)^{j}\left(\frac{\partial}{\partial y^{\prime}}\right)^{\gamma^{\prime}} \widetilde{a}\left(\lambda, y^{\prime}\right)\right| \leq C_{j \gamma^{\prime}}(1+\lambda)^{-\left[1+\left|\alpha_{1}\right|\right] / 2-j} .
$$


Mas, pela hipótese de indução, segue que

$$
\left|\lambda^{\left[1+\left|\alpha_{1}\right|\right] / 2} \int_{R^{n-1}} e^{i \lambda Q\left(y^{\prime}\right)} \widetilde{a}\left(\lambda, y^{\prime}\right)\left(y^{\prime}\right)^{\alpha^{\prime}} d y^{\prime}\right| \leq C(1+\lambda)^{-\left[(n-1)+\left|\alpha^{\prime}\right|\right] / 2},
$$

o que dá (2.24).

É análoga a prova se $|\alpha|$ é ímpar mais usando que se $\left|\alpha_{1}\right|$ é ímpar,

$$
\int_{-\infty}^{\infty} e^{i \lambda y_{1}^{2} / 2} a\left(\lambda, y_{1}, y^{\prime}\right) y_{1}^{\alpha_{1}} d y_{1}=O\left(\lambda^{-1 / 2-\left(\left|\alpha_{1}\right|+1\right) / 2}\right) \text {. }
$$

Prova do teorema 2.6 O Lema de Morse (ver apêndice) e (2.24) implicam o teorema 2.6, dado que podemos expressar a integral oscilatória

$$
I(\lambda)=\int e^{i \lambda \Phi(y)} a(\lambda, y) d y
$$

como

$$
I(\lambda)=\int e^{i \lambda Q(\widetilde{y})} \widetilde{a}(\lambda, \widetilde{y}) d \widetilde{y},
$$

onde $\tilde{a}$ é uma função que tem as mesmas propriedades que $a$ e $Q$ é uma forma quadrática como em (2.22). (De fato, estamos trapaceando, dado que esta mudança de variáveis somente se cumpre para uma vizinhança da origem, mas dado que $\nabla \Phi \neq 0$ quando $y \neq 0,(2.10)$ implica que sempre podemos assumir que o suporte de $a$ é suficientemente pequeno.)

Vamos ver uma versão do teorema 2.6 para coeficientes variáveis. Suponhamos que a função $\Phi(x, y) \in C^{\infty}$ é real e satisfaz

$$
\nabla_{y} \Phi(0,0)=0
$$

e

$$
\operatorname{det}\left(\frac{\partial^{2} \Phi}{\partial y_{j} \partial y_{k}}\right) \neq 0
$$

quando $x, y=0$. Então, pelo teorema da função implícita, existe uma função $y(x) \in C^{\infty}$ que é solução da equação

$$
\nabla_{y} \Phi(x, y(x))=0
$$

quando $x$ é pequeno. Dado que (2.25) mantém-se, $y(x)$ é chamado um ponto estacionário de $\Phi$. Para tal função consideramos integrais oscilatórias 


$$
I(x, \lambda)=\int e^{i \lambda \Phi(x, y)} \widetilde{a}(\lambda, x, y) d y,
$$

onde assumimos que $\widetilde{a}$ tem $y$-suporte suficientemente pequeno tal que $y(x)$ é a única solução de (2.25) e além disso, satisfaz

$$
\left|\left(\frac{\partial}{\partial \lambda}\right)^{\alpha}\left(\frac{\partial}{\partial x}\right)^{\gamma_{1}}\left(\frac{\partial}{\partial y}\right)^{\gamma_{2}} \widetilde{a}(\lambda, x, y)\right| \leq C_{\alpha \gamma}(1+\lambda)^{-|\alpha|}
$$

para todo $\alpha, \gamma_{j}$.

Corolário 2.2 Suponha que a e $\Phi$ são como acima. Então, para todo $\alpha=$ $0,1,2, \ldots$ e multi-índice $\gamma$

$$
\left|\left(\frac{\partial}{\partial \lambda}\right)^{\alpha}\left(\frac{\partial}{\partial x}\right)^{\gamma}\left(e^{-i \lambda \Phi(x, y(x))} I(x, \lambda)\right)\right| \leq C_{\alpha \gamma}(1+\lambda)^{-n / 2-|\alpha|} .
$$

Prova: Análoga ao corolário 2.1.

\section{Observações:}

1. Notemos que o Lema de Morse implica que

$$
\int_{-\infty}^{\infty} e^{i \lambda x^{2} / 2} d x=\left(\frac{\lambda}{2 \pi i}\right)^{-1 / 2}
$$

2. Suponha que

$$
Q(x)=1 / 2\left(x_{1}^{2}+\ldots+x_{j}^{2}-x_{j+1}^{2} \ldots-x_{n}^{2}\right),
$$

então $e^{i Q}$ é par. Podemos ver que (2.27) junto com (2.10) e o teorema 2.6 implicam que, para $\lambda>0$

$$
\int_{\mathbb{R}^{n}} e^{i \lambda Q(x)} \eta(x) d x=\left(\frac{\lambda}{2 \pi}\right)^{-n / 2} \eta(0) e^{\pi i / 4 s g n Q^{\prime \prime}}+\mathcal{O}\left(\lambda^{-n / 2-1}\right),
$$

onde $Q^{\prime \prime}$ denota o Hessiano de $Q$. Mas, por uso do Lema de Morse, temos que

$$
\int_{\mathbb{R}^{n}} e^{i \lambda \Phi(x)} \eta(x) d x=\left(\frac{\lambda}{2 \pi}\right)^{-n / 2} e^{i \lambda \Phi(0)} \eta(0)\left|\operatorname{det} \Phi^{\prime \prime}(0)\right|^{-1 / 2} e^{\pi i / 4 \operatorname{sgn} \Phi^{\prime \prime}}+\mathcal{O}\left(\lambda^{-n / 2-1}\right),
$$


se $\Phi$ tem um ponto crítico não degenerado em zero e $\eta$ tem suporte pequeno. Note que, neste caso, $\Phi^{\prime \prime}$ é constante sob supp $\eta$, também o primeiro termo sob o lado direito é bem definido.

\subsection{Operadores Pseudo-diferenciais}

\subsubsection{Definições}

Definição 2.4 Se $X \subset \mathbb{R}^{n}$ é um aberto. então um operador pseudo-diferencial em $X(\Psi D O)$ é um operador do tipo

$$
A u(x)=\iint e^{i \phi(x . y . \theta)} a(x, y, \theta) u(y) d y d \theta,
$$

com $u(y) \in C_{0}^{\infty}(X)$ e função de fase $\phi(x, y, \theta)=(x-y) \cdot \theta$. A classe de $(\Psi D O)$, definida por $a(x, y, \theta) \in S_{\rho, \delta}^{m}\left(X \times X \times \mathbb{R}^{n}\right)$ é denotada por $L_{\rho, \delta}^{m}(X)$ e $L^{-\infty}=\cap_{m} L_{1,0}^{m}$.

Definição 2.5 Sejam A um operador $(\Psi D O)$ com núcleo $K_{A}$ e supp $\left(K_{A}\right)$ o suporte de $K_{A}$. Considere as projeções $\pi_{1}: \operatorname{supp}\left(K_{A}\right) \mapsto X$ e $\pi_{2}: \operatorname{supp}\left(K_{A}\right) \mapsto Y$ obtidas por $(x, y) \mapsto x, \quad(x, y) \mapsto y$, respectivamente. Dizemos que A é propriamente suportado, se as projeções $\pi_{1}$ e $\pi_{2}$ são próprias, isto é, se $\{(x, y) \in$ $\operatorname{supp}\left(K_{A}\right), \quad x \in K \quad$ ou $\left.y \in K\right\}$ é compacto quando $K \subset X$ é compacto.

Definição 2.6 Seja A um $(\Psi D O)$ propriamente suportado. Seu símbolo é a função $\sigma_{A}(x, \xi)$ sobre $X \times \mathbb{R}^{n}$ tal que $\sigma_{A}(x, \xi)=e_{-\xi}(x) A e_{\xi}(x)$ onde $e_{\xi}(x)=e^{i x . \xi}$.

Definição $2.7 P^{*}$ indica o adjunto (ou transposto conjugado) de $P$, isto é $P^{*} u=\overline{P^{t} \bar{u}} e(P u, v)=\left(u, P^{*} v\right)$ se $(u, v)$ é o produto interno em $L^{2}\left(\mathbb{R}^{n}\right)$ e $u, v \in C_{0}^{\infty}(X)$.

As propriedades do adjunto de um operador pseudo-diferencial são obtidas por conjugação das propriedades do transposto. Por exemplo, se $\sigma(x, \xi)$ é um símbolo principal de um operador, então $\sigma(x,-\xi)$ é um símbolo principal do transposto e $\overline{\sigma(x, \xi)}$ é um símbolo principal do adjunto.

Definição 2.8 Seja $a_{j}(x, \theta) \in S_{\rho, \delta}^{m_{j}}\left(X \times \mathbb{R}^{n}\right), \quad j=1,2, \ldots$, com $m_{j} \rightarrow-\infty$ quando $j \rightarrow \infty$ e seja $a(x, \theta) \in C^{\infty}\left(X \times \mathbb{R}^{n}\right)$. Escrevemos

$$
a(x, \theta) \sim \sum_{j=1}^{\infty} a_{j}(x, \theta)
$$


se, para qualquer inteiro $r \geq K$, temos que

$$
\left[a(x, \theta)-\sum_{j=1}^{r-1} a_{j}(x, \theta)\right] \in S_{\rho, \delta}^{\bar{m}_{r}}
$$

onde

$$
\bar{m}_{r}=\max _{j \geq r} m_{j}
$$

\section{Lema 2.5 (Soma de Borel)}

Para uma sequência de símbolos $a_{j}(x, \theta) \in S_{\rho, \delta}^{m_{j}}\left(X \times \mathbb{R}^{n}\right), \quad j=1,2, \ldots$, com $m_{j} \rightarrow$ $-\infty$ quando $j \rightarrow \infty$, podemos sempre achar uma função $a(x, \theta) \in S_{\rho, \delta}^{m}, \quad m=$ $\max _{j}\left(m_{j}\right)$, tal que

$$
a \sim \sum_{j=1}^{\infty} a_{j}
$$

Se, adicionalmente, $a^{\prime} \in S_{\rho, \delta}^{m}$ é outra função com a mesma propriedade, isto é $a^{\prime} \sim \sum_{j=1}^{\infty} a_{j}$, então $a-a^{\prime} \in S^{-\infty}\left(X \times \mathbb{R}^{n}\right)$.

Prova: Vamos assumir que $m_{1}>m_{2}>m_{3}>\ldots$, se não for o caso, podemos sempre ter esta suposição com uma simples reorganização dos $m_{i}$ 's e juntando os termos de igual ordem. Seja $X=\cup_{j=1}^{\infty} X_{j}$, onde $X_{j}$ são conjuntos abertos de $X$ e tal que $K_{j}=\overline{X_{j}} \Subset X$ (i.e., $K_{j}$ é compacto em $\left.X\right)$. Seja $\phi(\theta) \in C^{\infty}\left(\dot{\mathbb{R}}^{n}\right)$ e colocamos

$$
a(x, \theta)=\sum_{j=1}^{\infty} \phi\left(\frac{\theta}{t_{j}}\right) a_{j}(x, \theta)
$$

onde $t_{j}$ aproxima-se ao $+\infty$ rapidamente quando $j \rightarrow+\infty$ tal que

$$
\left|\partial_{\theta}^{\alpha} \partial_{x}^{\beta}\left[\phi\left(\frac{\theta}{t_{j}}\right) a_{j}(x, \theta)\right]\right| \leq 2^{-j}(1+|\theta|)^{m_{j-1}-\rho|\alpha|+\delta|\beta|}
$$

para $x \in K_{l}$ e $|\alpha|+|\beta|+l \leq j$. Vamos provar que isto é sempre possível. Sabemos que

$$
\partial_{\theta}^{\alpha} \phi\left(\frac{\theta}{t}\right)=t^{-|\alpha|}\left(\partial_{\theta}^{\alpha} \phi\right)\left(\frac{\theta}{t}\right)
$$

e $|\theta| \leq t \leq 2|\theta|$ para $\theta \in \operatorname{supp}\left(\partial_{\theta}^{\alpha} \phi\left(\frac{\theta}{t}\right)\right)$, do qual obtemos

$$
\left|\partial_{\theta}^{\alpha} \phi\left(\frac{\theta}{t}\right)\right| \leq C_{\alpha}(1+|\theta|)^{-|\alpha|}, \quad t \geq 1
$$


onde $C_{\alpha}$ não depende de $t$, para $\phi\left(\frac{\theta}{t}\right)$ e $t \geq 1$ temos uniformemente em $t$ estimativas de classe $S_{1,0}^{0}$. Adicionalmente, de (2.31), segue que

$$
\left|\partial_{\theta}^{\alpha} \partial_{x}^{\beta}\left[\phi\left(\frac{\theta}{t}\right) a_{j}(x, \theta)\right]\right| \leq C_{j}(1+|\theta|)^{m_{j}-\rho|\alpha|+\delta|\beta|}
$$

se $x \in K_{l}, \quad t \geq 1 \mathrm{e}|\alpha|+|\beta|+l \leq j$. Observemos que

$$
(1+|\theta|)^{m_{j}-\rho|\alpha|+\delta|\beta|} \leq \epsilon(1+|\theta|)^{m_{j-1}-\rho|\alpha|+\delta|\beta|}
$$

para $(1+|\theta|)^{m_{j-1}-m_{j}} \geq \frac{1}{\epsilon}$. Assim, pela escolha de $t_{j}$, podemos obter (2.30) a partir do qual temos a convergência de (2.29), juntamente com todas as derivadas, uniformemente sob qualquer compacto $K \subset X$, e para $\alpha, \beta, l$ fixos arbitrários temos

$$
\left|\partial_{\theta}^{\alpha} \partial_{x}^{\beta}\left[\sum_{j=r+1}^{\infty} \phi\left(\frac{\theta}{t_{j}}\right) a_{j}(x, \theta)\right]\right| \leq 2^{-r}(1+|\theta|)^{m_{r}-\rho|\alpha|+\delta|\beta|},
$$

$x \in K_{l}, \quad|\alpha|+|\beta|+l \leq r$. Assim, $a-\sum_{j=1}^{r} a_{j} \in S_{\rho, \delta}^{m_{r}}\left(X \times \mathbb{R}^{n}\right)$ e dado que $a_{r} \in S_{\rho, \delta}^{m_{r}}\left(X \times \mathbb{R}^{n}\right)$, obtemos disto que $a-\sum_{j=1}^{r-1} a_{j} \in S_{\rho, \delta}^{m_{r}}\left(X \times \mathbb{R}^{n}\right)$. O método utilizado nesta prova é dito método da soma de Borel.

\subsubsection{Cálculo Pseudo-Diferencial}

Teorema 2.7 Suponhamos que $P(x, \xi) \in S^{m}$ e $Q(x, \xi) \in S^{n}$. Então $P(x, D) \circ$ $Q(x, D)$ é um operador pseudo-diferencial com simbolo $P \circ Q \in S^{m+n}$ dado por:

$$
(P \circ Q)(x, \xi) \sim \sum_{\alpha} \frac{1}{\alpha !} D_{\xi}^{\alpha} P(x, \xi)\left(\frac{\partial}{\partial x}\right)^{\alpha} Q(x, \xi) .
$$

Isto é,

$$
(P \circ Q)(x, \xi)-\sum_{|\alpha|<N} \frac{1}{\alpha !} D_{\xi}^{\alpha} P(x, \xi)\left(\frac{\partial}{\partial x}\right)^{\alpha} Q(x, \xi) \in S^{m+n-N} \quad \forall N .
$$

Prova: Notemos que o nucleo de $P(x, D) \circ Q(x, D)$ é

$$
\begin{aligned}
I(x, y) & =(2 \pi)^{-2 n} \iiint e^{i[\langle x-z, \eta\rangle+\langle z-y, \xi\rangle]} P(x, \eta) Q(z, \xi) d \eta d z d \xi \\
& =(2 \pi)^{-n} \int e^{i<x-y, \xi\rangle}(P \circ Q)(x, \xi) d \xi
\end{aligned}
$$


se

$$
\begin{aligned}
(P \circ Q)(x, \xi) & =(2 \pi)^{-n} \iint e^{i[\langle x-z, \eta\rangle+<z-x, \xi\rangle]} P(x, \eta) Q(z, \xi) d \eta d z \\
& =(\lambda / 2 \pi)^{n} \iint e^{i \lambda<(x-z),(\eta-\bar{\xi})>} P(x, \lambda \eta) Q(z, \xi) d \eta d z
\end{aligned}
$$

onde tomamos $\lambda=|\xi|$ e $\bar{\xi}=\xi / \lambda$. Notemos que a função de fase da última integral, $\Phi=<(x-z),(\eta-\bar{\xi})>$, satisfaz

$$
\nabla_{\eta, z} \Phi=(x-z, \bar{\xi}-\eta)
$$

Assim pelo lema 2.2, se $\rho(s) \in C_{0}^{\infty}(\mathbb{R})$ é um próximo a zero e é identicamente nulo quando $|s|>1 / 2$, vemos que, módulo uma função $C^{\infty}$ é rapidamente decrescente em $\xi$

$$
(P \circ Q)(x, \xi)=(\lambda / 2 \pi)^{n} \iint e^{i \lambda<(x-z),(\eta-\bar{\xi})>} \rho(|x-z|) \rho(1-|\eta|) P(x, \lambda \eta) Q(z, \xi) d \eta d z .
$$

Para estimar isto, usamos o método da fase estacionaria. Primeiro, notemos que (2.38) implica que o único ponto estacionario de $\Phi$ é $(\eta, z)=(\bar{\xi}, x)$. O Herssiano de $(\eta, z) \mapsto \Phi$ é a matriz $2 n \times 2 n$

$$
\left(\begin{array}{cc}
0 & -I_{n} \\
-I_{n} & 0
\end{array}\right)
$$

a qual tem determinante um. Assim, dado que $\Phi$ é identicamente nula no ponto estacionario e dado que a integração está sobre $\mathbb{R}^{2 n}$, vemos que a fórmula (2.28) implica que, módulo um símbolo em $S^{m+n-1}$,

$$
(P \circ Q)(x, \xi)=(\lambda / 2 \pi)^{-2 n / 2}(\lambda / 2 \pi)^{n} P(x, \lambda \bar{\xi}) Q(x, \xi)=P(x, \xi) Q(x, \xi)
$$

Assim, temos provado (2.33) para $N=1$. A prova da fórmula para o caso geral é similar excepto que precisamos usar a fórmula de Taylor

$$
P(x, \eta) Q(z, \xi)=\sum_{|\alpha|<N,|\beta|<N}\left[\frac{1}{\alpha !}\left(\frac{\partial}{\partial_{\xi}}\right)^{\alpha} P(x, \xi)(\eta-\xi)^{\alpha}\right] \times\left[\frac{1}{\beta !}\left(\frac{\partial}{\partial x}\right)^{\beta} Q(x, \xi)(z-x)^{\beta}\right]+R_{N}(x, z, \eta, \varepsilon
$$

Pelo teorema 2.6, fazendo uma análise análoga a $N=1$ vemos que,

$$
(2 \pi)^{-2 n} \iint e^{i<(x-z),(\eta-\xi)>} R_{N} d \eta d z \in S^{m+n-N},
$$


dado que $R_{N}$ é identicamente nulo para a ordem $2 N-1$ no ponto estacionario. Finalmente, usando o fato que

$$
\delta_{0}^{(k)}=(2 \pi)^{-1} \int e^{i t \xi}(i \xi)^{k} d \xi
$$

temos que

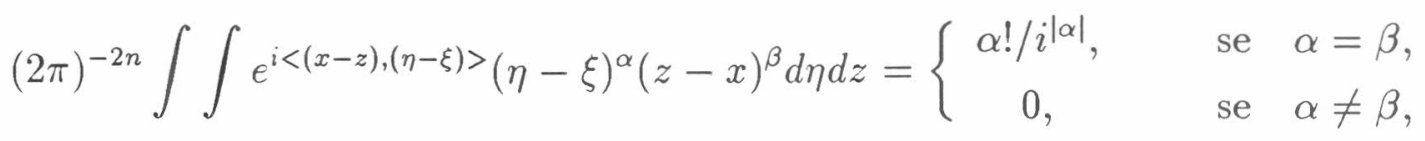

com esta observação $P \circ Q$ toma a forma (2.32). Logo pelo lema 2.3 de Van der Corput temos que $P \circ Q$ é um operador pseudo-diferencial com símbolo satisfazendo (2.33).

Teorema 2.8 Se P é um operador pseudo-diferencial de ordem $m$ então também é seu adjunto $P^{*}$. Além disso, o símbolo $P^{*}(x, D)$ é dado pela série

$$
P^{*}(x, \xi) \sim \sum_{\alpha} \frac{1}{\alpha !} \frac{\partial^{\alpha}}{\partial x} \overline{P(x, \xi)} .
$$

Prova: Vide (3), capítulo II, $\oint 4$.

Este teorema implica o seguinte fato importante que usaremos mais adiante.

Corolário 2.3 Se $P$ é um operador pseudo-diferencial auto-adjunto de ordem $m$, então o símbolo P satisfaz

$$
p(x, \xi)-\Re p(x, \xi) \in S^{m-1} .
$$

Lema 2.6 Sejam $a \in S_{\rho, \delta}^{0}\left(X \times \mathbb{R}^{n}\right) e$

$$
K=\left\{\varlimsup_{\lim _{k \rightarrow \infty}} a\left(x_{k}, \xi_{k}\right), \quad x_{k} \in X, \quad|\xi|_{k} \rightarrow \infty\right\} .
$$

Se $U$ é uma vizinhança de $K$ e $f \in C^{\infty}(U)$, então para alguma constante $C>0$ vale: $\phi(\xi) . f \circ a(x, \xi) \in S_{\rho, \delta}^{0}\left(X \times \mathbb{R}^{n}\right)$ donde $\phi \in C^{\infty}\left(\mathbb{R}^{n}\right)$ com $\phi(\xi)=1$ se $|\xi| \geq C+1$ e $\phi(\xi)=0$ se $|\xi| \leq C$.

Prova: Seja $b(x, \xi)=f \circ a(x, \xi)$. Por indução na ordem das derivadas, verificamos que

$$
\left|D_{x}^{\alpha} D_{\xi}^{\beta} b(x, \xi)\right| \leq C(K, \alpha, \beta)(1+|\xi|)^{-\rho|\beta|+\delta|\alpha|}
$$

para $|\xi|$ suficientemente grande. Portanto $\phi(\xi)$. foa $(x, \xi) \in S_{\rho, \delta}^{0}\left(X \times \mathbb{R}^{n}\right)$. 
Como aplicação do cálculo pseudo-diferencial temos que este simplifica questões de invertivilidade de operadores. No caso elíptico a construção da inversa é bastante fácil.

\section{Proposição 2.2 (Existência da parametriz elíptica)}

Seja $X \subset \mathbb{R}^{n}$ aberto. Se $A \in L_{\rho, \delta}^{m}(X)$ é elíptico, isto é, se o símbolo a $(x, \xi) \in S_{\rho, \delta}^{m}$ de $A$ é positivo então $A$ possui uma parametriz $B \in L_{\rho, \delta}^{-m}(X)$, isto é, existe $B \in L_{\rho, \delta}^{-m}(X)$ tal que $B A-I \in L^{-\infty}$ e $A B-I \in L^{-\infty}$, o mesmo operador $B$ serve como inversa à esquerda e à direita.

Prova: Como $A$ é elíptico podemos construir funções positivas, $C(x), \rho(x) \in C^{\infty}$ tais que

$$
|a(x, \xi)| \geq C(x)(1+|\xi|)^{m}, \quad x \in X,|\xi| \geq \rho(x) .
$$

Seja $\chi(t) \in C^{\infty}(\mathbb{R})$ tal que $\chi(t)=0$ se $t \leq 0, \quad \chi(t)=1$ se $t \geq 1$ e escrevemos $\phi(x, \xi)=\chi(|\xi|-\rho(x))$. Então, pelo Lema 2.6, temos que

$$
b_{0}(x, \xi)=\phi(x, \xi) / a(x, \xi) \in S_{\rho, \delta}^{-m}\left(X \times \mathbb{R}^{n}\right) .
$$

Seja $B_{0} \in L_{\rho, \delta}^{-m}$ propriamente suportado, com símbolo principal $b_{0}(x, \xi)$. Segue do cálculo que

$$
R_{0}=B_{0} A-I \in L_{\rho, \delta}^{-(\rho-\delta)}(X) .
$$

Construíremos por indução operadores propriamente suportados $B_{j} \in L_{\rho, \delta}^{-m-j(\rho-\delta)}$ tais que

$$
R_{k}=\sum_{j=0}^{k} B_{j} A-I \in L_{\rho, \delta}^{-(k+1)(\rho-\delta)} .
$$

Admitindo que $B_{0}, \ldots, B_{k-1}$ já foram construídos com as propriedades requeridas, devemos escolher $B_{k}$ de modo que

$$
R_{k}=R_{k-1}+B_{k} A \in L_{\rho, \delta}^{-(k+1)(\rho-\delta)} .
$$

Tomamos $b_{k}=-r_{k-1} b_{0} \in S_{\rho, \delta}^{-m-k(\rho-\delta)}$, vemos que $r_{k-1} b_{k} a=(1-\phi) r_{k-1} \in S^{-\infty}$ e vale (2.39). Tomando $B \sim \sum_{k=0}^{\infty} b_{k} \in L_{\rho, \delta}^{-m}$ obtemos $B A-I \in L^{-\infty}$. O mesmo argumento de fato implica que $A$ tem parametriz direita $\bar{B} \in L^{-m}$, isto é, $A \bar{B}-I \in L^{-\infty}$. Vejamos que $\bar{B}=B$, de fato, $\bar{B}=(B A) \bar{B}=B(A \bar{B})=B$ módulo $L^{-\infty}$. 
Seja $P\left(x, t, D_{x}, D_{t}\right)$ um operador diferencial parcial de ordem $m$ com símbolo principal $p(x, t, \xi, \tau)$. Suponhamos que $p\left(x_{0}, t_{0}, \xi_{0}, \tau\right)$ tem uma raiz simples $\tau_{0}$ em $\left(x_{0}, t_{0}, \xi_{0}\right), \quad \xi_{0} \in \mathbb{R}^{n} \backslash\{0\}$, isto é,

$$
p\left(x_{0}, t_{0}, \xi_{0}, \tau_{0}\right)=0, \quad \frac{\partial}{\partial \tau} p\left(x_{0}, t_{0}, \xi_{0}, \tau_{0}\right) \neq 0 .
$$

Então para $(x, t, \xi)$ numa vizinhança de $\left(x_{0}, t_{0}, \xi_{0}\right)$ podemos fatorar

$$
p=(\tau-\lambda(x, t, \xi)) q_{m-1}(x, t, \xi, \tau), \quad q_{m-1}(x, t, \xi, \tau) \neq 0
$$

com $\lambda$ homogêneo de grau 1 em $\xi$, e $q_{m-1}$ um polinômio em $\tau$ de grau $m-1$ e homogêneo em $(\xi, \tau)$. Com $\lambda$ e $q_{m-1}$ convenientemente estendidos, o produto de operadores

$$
\left(D_{t}-\lambda\left(x, t, D_{x}\right)\right) q_{m-1}\left(x, t, D_{x}, D_{t}\right)
$$

é uma aproximação de $P\left(x, t, D_{x}, D_{t}\right)$, o operador $q_{m-1}(x, t, \xi, \tau)$ é elíptico em $\left(x_{0}, t_{0}, \xi_{0}, \tau_{0}\right)$ e se é elíptico para todo $(x, t, \xi, \tau)$ então é localmente invertível, no caso onde $\lambda$ é real para todo $\xi_{0} \in \mathbb{R}^{n} \backslash\{0\}$,

$$
D_{t}-\lambda\left(x, t, D_{x}\right)
$$

é um operador hiperbólico. Vamos ver um resultado mais preciso deste tipo de fatoração de operadores o qual vai ser usado no capítulo 6 para concluir a prova do teorema de propagação de singularidades.

\section{Proposição 2.3 (fatorização micro-local hiperbolica)}

Existe um operador

$$
Q\left(x, t, D_{x}, D_{t}\right)=\sum_{j=0}^{m-j} a_{j}\left(x, t, D_{x}\right) D_{t}^{j},
$$

onde para cada $j, a_{j}$ é um operador pseudo-diferencial nas variáveis $x$ de ordem $m-1-j$, além disso existe um operador pseudo-diferencial nas variáveis $x, \rho\left(x, t, D_{x}\right)$ de ordem um, com símbolo principal $\lambda(x, t, \xi)$ numa vizinhança de $\left(x_{0}, t_{0}, \xi_{0}\right)$, tal que o simbolo de

$$
P\left(x, t, D_{x}, D_{t}\right)-\left(D_{t}-\rho\left(x, t, D_{x}\right)\right) Q\left(x, t, D_{x}, D_{t}\right)
$$

é identicamente nulo para $(x, t, \xi)$ numa vizinhança de $\left(x_{0}, t_{0}, \xi_{0}\right)$ e para todo $\tau$. 
Prova: Precisamos determinar os símbolos de $Q$ e $\rho$ para $(x, t, \xi)$ próximos de $\left(x_{0}, t_{0}, \xi_{0}\right)$ e para todo $\tau$; escrevemos os símbolos

$$
Q(x, t, \xi, \tau) \sim q_{m-1}+q_{m-2}+\ldots+q_{0}+q_{-1}+\ldots
$$

e

$$
\rho(x, t, \xi)=\lambda(x, t, \xi)+\rho_{0}+\rho_{-1}+\ldots
$$

onde os $q_{j}$ são homogêneos em $(\xi, \tau)$ de grau $j$ e polinomiais em $\tau$, e os $\rho_{j}$ são homogêneos em $\xi$ de grau $j$. Para achar o símbolo podemos simplesmente compor os operadores correspondentes e comparar o símbolo resultante da composição $\sum_{\alpha} \frac{1}{\alpha !}\left[\partial_{\xi}^{\alpha}\left(D_{t}-\rho(x, t, \xi)\right)\right]\left[D_{x}^{\alpha} Q(x, t, \xi, \tau)\right] \operatorname{com} p(x, t, \xi, \tau)$, e igualar fora os termos correspondentes. Por exemplo, olhando os termos de grau $(m-1)$ achamos

$$
-\rho_{0} q_{m-1}+D_{t} q_{m-1}-\sum \frac{\partial \lambda}{\partial \xi_{j}} D_{x^{\prime}} q_{m-1}+(\tau-\lambda) q_{m-2}=p_{m-1} .
$$

Tomando $\tau=\lambda(x, t, \xi)$ achamos

$$
\rho_{0}=\left.\frac{D_{t} q_{m-1}-\sum \lambda_{\xi_{\jmath}} D_{x^{\jmath}} q_{m-1}-p_{m-1}}{q_{m-1}}\right|_{\tau=\lambda(x, t, \xi)}
$$

substituindo $\rho_{0}$ em (2.40) determinamos $q_{m-2}$, como um polinômio em $\tau$, e analogamente os outros termos.

\subsubsection{Continuidade em $L^{2}$ dos Operadores Pseudo-Diferenciais}

Nesta seção, demonstraremos o teorema de continuidade em $L^{2}$ dos Operadores Pseudo-Diferenciais que mais adiante será usado para provar a continuidade em $L^{2}$ dos Operadores integrais de Fourier (FIO).

\section{Lema 2.7 (Schur)}

Se $K$ é uma função contínua em $\mathbb{R}^{n} \times \mathbb{R}^{n}$ e

$$
\sup _{y} \int|K(x, y)| d x \leq C, \quad \sup _{x} \int|K(x, y)| d y \leq C,
$$

então o operador integral com kernel $K$ tem norma $\leq C$ em $L^{2}\left(\mathbb{R}^{n}\right)$. 
Prova: Pela desigualdade de Cauchy-Schwarz temos

$$
|K u(x)|^{2} \leq \int|K(x, y)||u(y)|^{2} d y \int|K(x, y)| d y .
$$

Se a última integral é estimada por $C$, uma integração com respeito a $x$ implica que

$$
\int|K u(x)|^{2} d x \leq C \iint|K(x, y)||u(y)|^{2} d x d y \leq C^{2} \int|u(y)|^{2} d y<\infty .
$$

Teorema 2.9 Todo operador pseudo-diferencial $A=a(x, D): L^{2}\left(\mathbb{R}^{n}\right) \rightarrow L^{2}\left(\mathbb{R}^{n}\right)$, é um operador limitado se $a \in S^{-n-1}$.

Prova: Pela fórmula do kernel de $A$ segue que, neste caso, o kernel $K(x, y)$ do operador $A$ é limitado por

$$
|K(x, y)| \leq \int|a(x, \xi)| d \xi \leq C \int(1+|\xi|)^{-n-1} d \xi \leq C<\infty .
$$

O comutador de $A$ multiplicado por $x,[x, a(x, D)]=-a^{(j)}(x, D)$, também pertence a $S^{-n-1}$, o kernel é $(x-y) K(x, y)$, e também por (2.41) é limitado. Repetindo $n+1$ vezes concluímos que

$$
(1+|x-y|)^{n+1} K(x, y) \leq C \quad \forall x, y .
$$

Segue que $K(x, y)$ satisfaz a hipótese do Lema de Schur 2.7, e também é limitado de $L^{2} \rightarrow L^{2}$. Pela definição da norma de $L^{2}\left(\mathbb{R}^{n}\right)$

$$
\|a(x, D) u\|^{2}=(A u, A u)=\left(u, A^{*} A u\right)=(u, b(x, D) u)
$$

onde

$$
b(x, D)=a^{*}(x, D) a(x, D) .
$$

Suponha que $a \in S^{-\frac{n+1}{2}}$. Então $b \in S^{-(n+1)}$ e é limitado com

$$
\|b(x, D) u\| \leq C\|u\| .
$$

Por (2.43) e Cauchy-Schwarz temos que

$$
\|a(x, D) u\|^{2} \leq\|u\|\|\mid b(x, D) u\| \leq C\|u\|^{2} .
$$

Repetindo este argumento deduzimos que quando $a$ é de classe $S^{-k}$, qualquer que seja $k>0, a(x, D)$ é limitado. 
Teorema 2.10 Sejam $X \subset \mathbb{R}^{n}$ um aberto, $A \in L_{\rho, \delta}^{0}(X), \quad \delta<\rho$. Então

$$
A: L_{c}^{2}(X) \rightarrow L_{l o c}^{2}(X)
$$

é um operador contínuo.

Prova: Para provar a continuidade em $L_{l o c}^{2}(X)$ é suficiente provar que dado um compacto $K \subset X$ e $\phi \in C_{0}^{\infty}(X)$ existe $L>0$ tal que

$$
\|\phi A u\|_{L^{2}} \leq L\|u\|_{L^{2}}, \quad u \in C_{0}^{\infty}(K) .
$$

Seja $A$ propriamente suportado, $a$ um símbolo de $A, a \in S_{\rho, \delta}^{0}\left(X \times \mathbb{R}^{n}\right)$. Então $a_{1}(x, \xi)=\phi(x) a(x, \xi)$ é um símbolo de $A_{1}=\phi A$ e existe $M>0$ tal que

$$
\left|a_{1}(x, \xi)\right|<M, \quad x, \xi \in \mathbb{R}^{n} .
$$

Construiremos um operador $B \in L_{\rho, \delta}^{0}(X)$, propriamente suportado, tal que

$$
A_{1}^{*} A_{1}+B^{*} B-M^{2} I \in L^{-\infty}(X),
$$

onde $I$ indica a identidade, $I u=u$ e $A_{1}^{*}\left(B^{*}\right)$ indica o adjunto (ou transposto conjugado) de $A_{1}(B)$ respectivamente. O operador $C=M^{2} I-A_{1}^{*} A_{1}$ é autoadjunto $\left(C^{*}=C\right)$, propriamente suportado e possui um símbolo principal

$$
c(x, \xi)=M^{2}-\overline{a_{1}(x, \xi)} a_{1}(x, \xi)=M^{2}-\left|a_{1}\right|^{2}>0 .
$$

seja $b_{0}(x, \xi)=(c(x, \xi))^{1 / 2}>0$. Aplicando o Lema $2.6 \operatorname{com} f(z)=z^{1 / 2}$, vemos que $b_{0}(x, \xi) \in S_{\rho, \delta}^{0}(X)$. Seja $B_{0} \in L_{\rho, \delta}^{0}(X)$, propriamente suportado, com símbolo $b_{0}$. Usando o cálculo concluímos que

$$
C-B_{0}^{*} B_{0} \in L_{\rho, \delta}^{\delta-\rho}(X)
$$

Suponhamos indutivamente já ter construído $B_{j} \in L_{\rho, \delta}^{j(\delta-\rho)}(X), \quad j=1 \ldots k-1$, propriamente suportados e tais que

$$
R_{k}=C-\left(B_{0}+\ldots+B_{k-1}\right)^{*}\left(B_{0}+\ldots+B_{k-1}\right) \in L_{\rho, \delta}^{k(\delta-\rho)}(X)
$$


e desejamos construir $B_{k} \in L_{\rho, \delta}^{k(\delta-\rho)}(X)$, propriamente suportado e tal que

$$
\begin{aligned}
R_{k+1} & =C-\left(B_{0}+\ldots+B_{k}\right)^{*}\left(B_{0}+\ldots+B_{k}\right) \\
& \equiv R_{k}-B_{k}^{*} B_{0}-B_{0}^{*} B_{k} \equiv 0 \quad(\bmod L)_{\rho, \delta}^{(k+1)(\delta-\rho)}
\end{aligned}
$$

Seja $r_{k} \in S_{\rho, \delta}^{k(\delta-\rho)}$ um símbolo de $R_{k}$. Como $R_{k}=R_{k}^{*}$, verifica-se que $\Im r_{k} \in$ $S_{\rho, \delta}^{(k+1)(\delta-\rho)}$ e concluímos, pelo corolário 2.3, que $\Re r_{k}$ também é um símbolo principal de $R_{k}$. Basta definir

$$
2 b_{k}(x, \xi)=\Re r_{k}(x, \xi) / b_{0}(x, \xi) \in S_{\rho, \delta}^{k(\delta-\rho)}
$$

e tomar $B_{k}$ propriamente suportado com símbolo $b_{k}$ para obter (2.44). Tomando agora $b \sim \sum_{j=1}^{\infty} b_{j}$ pelo lema 2.5 e $B$ propriamente suportado com símbolo $b(x, \xi)$ obtemos (2.44). Se $R$ é operador definido por (2.44) temos

$$
\left\|A_{1} u\right\|_{L^{2}}^{2} \leq\left(A_{1} u, A_{1} u\right)+(B u, B u)=M^{2}\|u\|_{L^{2}}^{2}+(R u, u) .
$$

Como $R$ é regularizante e propriamente suportado, pelo teorema 2.9 temos que para algum compacto $K^{\prime} \Subset X$

$$
|(R u, u)| \leq C(K)\|u\|_{L^{2}}^{2}, \quad u \in C_{0}^{\infty}(K) .
$$




\section{Capítulo 3}

\section{Conjunto Frente de Ondas}

O conjunto frente de ondas de uma distribuição é um refinamento microlocal da noção de suporte singular. O objetivo final deste capítulo consistirá em descrever detalhadamente este conjunto para as soluções fundamentais de operadores de onda em $\mathbb{R}^{n+1}$.

Definição 3.1 Sejam $X \subset \mathbb{R}^{n}$ um aberto, $\left(x_{0}, \xi_{0}\right) \in X \times\left(\mathbb{R}^{n} \backslash\{0\}\right)$ e u $\in$ $\mathcal{D}^{\prime}(X)$. Dizemos que $\left(x_{0}, \xi_{0}\right)$ não pertence ao conjunto frente de ondas de $u$ (denotado $\left.\left(x_{0}, \xi_{0}\right) \notin W F(u)\right)$ se e somente se existem um cone aberto $\Gamma \subset \mathbb{R}^{n}$ contendo $\xi_{0}, v \in \mathcal{E}^{\prime}(X)$ e constantes $C_{N}$ tais que $u=v$ em uma vizinhança de $x_{0}, e$

$$
|\hat{v}(\xi)| \leq C_{N}(1+|\xi|)^{-N} \quad \forall \quad \xi \in \Gamma, \quad N=1,2, \ldots
$$

i.e., $\hat{v}(\xi)$ é rapidamente decrescente numa vizinhança cônica de $\xi_{0}$.

Lema 3.1 Se $\phi \in C_{0}^{\infty}\left(\mathbb{R}^{n}\right)$ e $v \in \mathcal{E}^{\prime}\left(\mathbb{R}^{n}\right)$ então

$$
W F(\phi v) \subset W F(v)
$$

i.e, se $\left(x_{0}, \xi_{0}\right) \notin W F(v)$ então $\left(x_{0}, \xi_{0}\right) \notin W F(\phi v)$.

Prova: A transformada de Fourier de $u=\phi v$ é a convolução

$$
\hat{u}(\xi)=(2 \pi)^{-n} \hat{\phi} * \hat{v}=(2 \pi)^{-n} \int \hat{\phi}(\eta) \hat{v}(\xi-\eta) d \eta,
$$


onde $\phi$ é rapidamente decrescente e $|\hat{v}(\xi)| \leq C(1+|\xi|)^{N}$ para algum $N$. Seja $0<c<1$ e dividindo a integral em duas partes tais que $|\eta|<c|\xi|$ e $|\eta| \geq c|\xi|$, temos

$$
\hat{u}(\xi)=(2 \pi)^{-n} \int_{|\eta|<c|\xi|} \hat{\phi}(\eta) \hat{v}(\xi-\eta) d \eta+(2 \pi)^{-n} \int_{|\eta| \geq c|\xi|} \hat{\phi}(\eta) \hat{v}(\xi-\eta) d \eta .
$$

No segundo caso $|\xi-\eta| \leq|\xi|+|\eta| \leq c^{-1}|\eta|+|\eta|=\left(1+c^{-1}\right)|\eta| \mathrm{e}$

$$
1+|\xi| \leq\left(1+c^{-1}\right)+\left(1+c^{-1}\right)|\eta|=\left(1+c^{-1}\right)(1+|\eta|) \text {. Daí }
$$

$$
\begin{aligned}
(2 \pi)^{n}|\hat{u}(\xi)| & \leq \sup _{|\xi-\eta|<c|\xi|}|\hat{v}(\eta)|\|\hat{\phi}\|_{L^{1}}+C \int_{|\eta|>c|\xi|}|\hat{\phi}(\eta)|(1+|\xi-\eta|)^{M} d \eta \\
& \leq \sup _{|\xi-\eta|<c|\xi|}|\hat{v}(\eta)|\|\hat{\phi}\|_{L^{1}}+C \int_{|\eta|>c|\xi|}|\hat{\phi}(\eta)|\left(1+c^{-1}\right)^{M}(1+|\eta|)^{M} d \eta .
\end{aligned}
$$

Se $\Gamma$ é um cone aberto onde $|\hat{v}(\xi)| \leq C_{N}(1+|\xi|)^{-N}$ e $\Gamma_{1}$ é um cone fechado tal que $\Gamma_{1} \subset \Gamma \cup\{0\}$ podemos escolher $c$ tal que $\eta \in \Gamma$ se $\xi \in \Gamma_{1}$ e $|\xi-\eta|<c|\xi|$, pois isto é certo quando $|\xi|=1$. Desde que $|\xi|-|\eta| \leq|\xi-\eta|<c|\xi|$ então $1+|\eta| \geq(1-c)(1+|\xi|)$ e segue que $\hat{u}$ é rapidamente decrescente em $\Gamma_{1}$. De fato, temos, para $N \geq 0$, que

$$
\begin{aligned}
\sup _{\Gamma_{1}}(1+|\xi|)^{N}|\hat{u}(\xi)| & \leq(1+|\xi|)^{N} \quad \sup _{\Gamma}|\hat{v}(\eta)|(1+|\eta|)^{N}\|\hat{\phi}\|_{L^{1}} \\
& +C(1+|\xi|)^{N} \int|\hat{\phi}(\eta)|(1+|\eta|)^{M}\left(1+c^{-1}\right)^{M} d \eta . \\
& \leq(1-c)^{-N} \sup _{\Gamma}|\hat{v}(\eta)|(1+|\eta|)^{N}\|\hat{\phi}\|_{L^{1}} \\
& +C\left(1+c^{-1}\right)^{N+M} \int|\hat{\phi}(\eta)|(1+|\eta|)^{N+M} d \eta .
\end{aligned}
$$

Como $\hat{v}$ e $\hat{\phi}$ são de decrescimento rápido em $\Gamma$ então

$$
\sup _{\Gamma_{1}}(1+|\xi|)^{N}|\hat{u}(\xi)| \leq C_{N} .
$$

Observação: Na definição 3.1 podemos tomar $v=\phi u$ para $\phi(x) \in C_{0}^{\infty}(X)$. Escolhemos $v$ como na definição, $\phi=1$ numa vizinhança de $x_{0}$ e $\phi u=\phi v$. Logo aplicamos o Lema 3.1. Exemplos:

1. Seja $\delta$ a função Delta de Dirac. Sabemos que o suporte singular suppsing $(\delta)=$ $\{0\}$ e que $\widehat{\phi \delta}(\xi)=\int e^{-i x \xi}(\phi \delta)(x) d x=\phi(0)$. Então

$$
|\widehat{\phi \delta}(\xi)|=|\phi(0)|
$$


como $|\phi(0)|$ não decresce rapidamente para todo $\xi \neq 0$, então

$$
W F(\delta)=\{0\} \times\left(\mathbb{R}^{n} \backslash\{0\}\right) .
$$

2. Seja $n=2, u(x)=Y\left(x_{1}\right) \times 1_{x_{2}}$. Notemos que

$$
\operatorname{suppsing}(u)=\left\{\left(0, x_{2}\right): x_{2} \in \mathbb{R}\right\} .
$$

Vemos que

$$
W F(u)=\left\{\left(0, x_{2}\right): x_{2} \in \mathbb{R}\right\} \times\left\{\left(\xi_{1}, 0\right): \xi_{1} \neq 0\right\} .
$$

De fato, observemos que

$$
W F(u) \subseteq\left\{\left(0, x_{2}\right): x_{2} \in \mathbb{R}\right\} \times\left(\mathbb{R}^{2} \backslash\{0\}\right) .
$$

Seja $x_{2}^{\prime} \in \mathbb{R}$. Bastará mostrar que se $\xi_{2}^{\prime} \neq 0$ então $\left(0, x_{2}^{\prime}, \xi_{1}^{\prime}, \xi_{2}^{\prime}\right) \notin W F(u)$. Seja $\phi_{1} \in C_{0}^{\infty}(\mathbb{R}), \phi_{1}=1$ em uma vizinhança da origem e seja $\phi_{2} \in C_{0}^{\infty}(\mathbb{R})$, $\phi_{2}=1$ em uma vizinhança de $x_{2}^{\prime}$. Definimos

$$
\phi\left(x_{1}, x_{2}\right)=\phi_{1}\left(x_{1}\right) \phi_{2}\left(x_{2}\right)=\left(\phi_{1} \times \phi_{2}\right)\left(x_{1}, x_{2}\right)
$$

$\log 0$

$$
\widehat{\phi u}\left(\xi_{1}, \xi_{2}\right)=\phi_{1} \widehat{Y \times} \phi_{2}\left(\xi_{1}, \xi_{2}\right)=\hat{\phi}_{2}\left(\xi_{2}\right) \int_{0}^{\infty} \phi_{1}\left(x_{1}\right) e^{-i \xi_{1} x_{1}} d x_{1} .
$$

Portanto,

$$
\left|\widehat{\phi u}\left(\xi_{1}, \xi_{2}\right)\right| \leq C\left|\hat{\phi}_{2}\left(\xi_{2}\right)\right| \leq C_{N}\left(1+\left|\xi_{2}\right|\right)^{-N}, \forall\left(\xi_{1}, \xi_{2}\right) .
$$

Suponhamos $\xi_{2}^{\prime} \neq 0$, em particular $\xi_{2}^{\prime}>0$. Seja $0<\delta<\frac{\xi_{2}^{\prime}}{\xi_{1}^{\prime}}$ e tomemos

$$
\Gamma=\left\{\left(\xi_{1}, \xi_{2}\right): \xi_{2}>\delta\left|\xi_{1}\right|\right\} .
$$

Temos $\left(\xi_{1}^{\prime}, \xi_{2}^{\prime}\right) \in \Gamma$ e $\left(\xi_{1}, \xi_{2}\right) \in \Gamma$ então $|\xi| \leq\left(1+\frac{1}{\delta}\right)^{1 / 2} \xi_{2}$ logo $\left(\xi_{1}, \xi_{2}\right) \in \Gamma$ implica $\left|\widehat{\phi u}\left(\xi_{1}, \xi_{2}\right)\right| \leq C_{N}(1+|\xi|)^{-N}$. O caso $\xi_{2}^{\prime}<0$ é análogo.

3. Seja $T_{\alpha}$ a transformada de Fourier inversa de $(2 \pi)^{-1 / 2}\left(1+|\xi|^{2}\right)^{\alpha}$ em $\mathcal{S}^{\prime}(\mathbb{R})$. Se $\alpha$ é um inteiro positivo $n$, então $T_{n}=\sum_{m=0}^{n}(-1)^{m}\left(\begin{array}{c}n \\ m\end{array}\right) \delta^{(2 m)}(x)$. Por (1) temos que

$$
W F\left(T_{n}\right)=\{(0, \gamma) / \gamma \neq 0\} .
$$


4. Sejam $x_{1}, x_{2}$ coordenadas em $\mathbb{R}^{2}$ e sejam $\delta\left(x_{1}\right), \delta\left(x_{2}\right)$ definidas no caminho usual

$$
\int f\left(x_{1}, x_{2}\right) \delta\left(x_{1}\right) d x_{1} d x_{2}=\int f\left(0, x_{2}\right) \delta\left(x_{2}\right)
$$

e

$$
\int f\left(x_{1}, x_{2}\right) \delta\left(x_{2}\right) d x_{1} d x_{2}=\int f\left(x_{1}, 0\right) \delta\left(x_{1}\right) .
$$

Sabemos que o suporte singular suppsing $\left(\delta\left(x_{1}\right)\right)=\left\{\left(0, x_{2}\right) / x_{2} \in \mathbb{R}\right\} \mathrm{e}$ $\widehat{\phi \delta_{x_{1}}}(\xi)=\int e^{-i x \xi}\left(\phi \delta_{x_{1}}\right)(x) d x=\int e^{-i x_{2} \xi_{2}} \phi\left(0, x_{2}\right) d x_{2}$. Como $\phi \in C_{0}^{\infty}$ então $\left|\widehat{\phi \delta_{x_{1}}}(\xi)\right| \leq C_{M}\left(1+\left|\xi_{2}\right|\right)^{-M} \quad M=1,2 \ldots$ e portanto

$$
W F\left(\delta\left(x_{1}\right)\right)=\left\{\left(0, x_{2}, \gamma, 0\right) / x_{2} \in \mathbb{R}, \gamma \neq 0\right\} .
$$

Analogamente

$$
W F\left(\delta\left(x_{2}\right)\right)=\left\{\left(x_{1}, 0,0, \gamma\right) / x_{1} \in \mathbb{R}, \gamma \neq 0\right\}
$$

5. Seja $\phi \in C_{0}^{\infty}, \hat{\phi} \geq 0$ e $\hat{\phi}(0)=1$. Se $\theta \neq 0$ então $u(x)=\sum_{1}^{\infty} k^{-2} \phi(k x) e^{i k^{2}<x, \theta>}$ é uma função tal que $W F(u)=\{(0, t \theta), t>0\}$. De fato, sabemos que todo termo de u está em $C^{\infty}$ e o suporte tende para zero, então temos que suppsing $(u) \subset\{0\}$. Agora,

$$
\hat{u}(\xi)=\sum_{1}^{\infty} k^{-2-n} \hat{\phi}\left(\left(\xi-k^{2} \eta\right) / k\right) .
$$

Além sisso, $\hat{u}\left(k^{2} \eta\right) \geq k^{-2-n}$ e daí podemos concluir que

$$
\{(0, t \theta), t>0\} \subset W F(u) .
$$

Verifiquemos que $W F(u) \subset\{(0, t \theta), t>0\}$. Se $V$ é um cone aberto contendo $\eta$ então $|\xi-t \eta| \geq C(|\xi|+t)$ se $\xi \notin V$ e $t \geq 0$ (isto é certo quando $|\xi|+t=1)$. Dai, $\left|\xi-k^{2} \eta\right| / k \geq C\left(|\xi|+k^{2}\right) / k \geq C(|\xi|)^{1 / 2}$ e portanto, $\hat{u}$ é de decrescimento rápido em $C V$.

O seguinte lema será usado no último capítulo para provar o teorema de propagação de singularidades.

Lema 3.2 Um ponto $\left(x_{0}, \xi_{0}\right)$ não está no conjunto frente de ondas de u (com suporte compacto) se e só se existe um operador pseudo-diferencial $A$ de grau zero com símbolo principal $a(x, \xi)$ tal que

$$
a\left(x_{0}, \xi_{0}\right) \neq 0 \quad \text { e } A u \in C^{\infty} .
$$


Prova: $\Rightarrow$ Suponhamos $\left(x_{0}, \xi_{0}\right) \notin W F u$, tal que $u=v+w$ com $v$ satisfazendo $\hat{v}(\xi)=O\left(|\xi|^{-N}\right) \quad \forall N$ em $\Gamma$. Seja $A$ um operador pseudo-diferencial com símbolo $\zeta(x) \chi(\xi)$ onde $\chi$ é homogênea de grau zero para $|\xi|>1$ com suporte em $\Gamma$, e $\chi\left(\xi_{0}\right) \equiv 1 ; \zeta$ tem suporte próximo de $x_{0}$ tal que $\zeta \equiv 0$ sobre o suppw. Então

$$
A u=A v+A w
$$

com $A w$ uma função $C^{\infty}$, enquanto que

$$
A v(x)=\int e^{i x \cdot \xi} \zeta(x) \chi(\xi) \hat{v}(\xi) d \xi=\zeta(x)(\chi(\xi) \hat{v}(\xi))^{\vee}
$$

a qual por hipótese tem decrescimento no infinito mais rápido que qualquer potência de $|\xi|$; assim $A v \in C^{\infty}$.

$\Leftarrow$ Suponhamos que existe um operador $A$ satisfazendo (3.3). Assim, $A$ é elíptico em $\left(x_{0}, \xi_{0}\right)$ e, pela proposição 2.2 da parametriz, podemos construir um operador pseudo-diferencial $Q$ tal que o símbolo de $Q A$ é identicamente um (para $|\xi|>1)$ numa vizinhança $\Omega$ definida por

$$
\Omega:\left\{\left|x-x_{0}\right|<\epsilon \text { e } \xi \in \Gamma \text {, tal que } \Gamma \text { é um cone em } \xi_{0}\right\} .
$$

Seja $v=\zeta u$, onde $\zeta(x) \in C_{0}^{\infty} \operatorname{com} \zeta \equiv 1$ para $\left|x-x_{0}\right|<\frac{\epsilon}{4}$ e $\zeta \equiv 0$ para $\left|x-x_{0}\right|>\frac{\epsilon}{2}$. Seja $\chi(\xi)$ uma função $C^{\infty}$, homogênea de grau zero para $|\xi| \cdot>1$, com suporte compacto em $\Gamma$, e $\chi \equiv 1$ numa vizinhança de $\xi_{0}$. Vamos verificar que $\chi(D) v$ é uma função $C^{\infty}$.

$$
\begin{aligned}
\chi(D) v & =Q A \chi(D) \zeta u+(I-Q A) \chi(D) v \\
& =\chi(D) \zeta Q A u+[Q A, \chi(D) \zeta] u+(I-Q A) \chi(D) v .
\end{aligned}
$$

Pela construção de $Q$ e $\chi$ vemos que o símbolo de $(I-Q A) \chi(D)$ é identicamente nulo e portanto $(I-Q A) \chi(D) v$ é uma função $C^{\infty}$. Analogamente para o símbolo de $[Q A, \chi(D) \zeta]$, e também $[Q A, \chi(D) \zeta]$ u é uma função $C^{\infty}$. Finalmente, dado que $A u$ é $C^{\infty}$ temos que, por hipótese, também o é $\chi(D) \zeta Q A u$ e temos que $\chi(D) v$ é $C^{\infty}$. Para concluir a prova do lema, observe que, pela definição de conjunto frente de ondas basta só provar que $\chi(D) \hat{v}(\xi)=O\left(|\xi|^{-N}\right)$ para todo $N$ com $|\xi| \rightarrow \infty$. Isto é uma consequência imediata do seguinte lema.

Lema 3.3 Seja $v \in \mathcal{E}^{\prime}\left(\mathbb{R}^{n}\right), \quad \chi(\xi) \in S_{\rho, 0}^{m}$. Então para $\rho(x$, suppv $) \geq 1$ temos

$$
\left|D^{\alpha} \chi(D) v(x)\right| \leq C_{\alpha, N}|x|^{-N}
$$


Prova: Dado que $\zeta^{\alpha} \chi(\xi) \in S_{\rho, 0}^{m+|\alpha|}$, tudo reduz-se ao caso $\alpha=0$. Além disso, dado que $v=\sum_{|\alpha| \leq m} D^{\alpha} v_{\alpha} \operatorname{com} v_{\alpha}$ 's contínuos, podemos reduzir ao caso onde $v$ é contínua. Temos

$$
\chi(D) v(x)=\iint e^{i(x-y) \cdot \xi} \chi(\xi) v(y) d y d \xi .
$$

Integrando por partes e usando a fórmula

$$
|x-y|^{-2 N}(-\Delta \xi)^{N} e^{i(x-y) \cdot \xi}=e^{i(x-y) \cdot \xi}
$$

temos que

$$
\chi(D) v(x)=\iint e^{i(x-y) \cdot \xi}\left((-\Delta \xi)^{N} \chi(\xi)\right)|x-y|^{-2 N} v(y) d y d \xi
$$

o qual tem sentido para $\rho(x$, suppv $) \geq 1$. Fazendo $N$ tão grande que $(-\Delta \xi)^{N} \chi(\xi) \in$ $S_{\rho, 0}^{-n-1}$, vemos que a integral (3.4) converge absolutamente e

$$
|\chi(D) v(x)| \leq C<x>^{-2 N} \quad \text { para } \quad \rho(x, \text { suppv }) \geq 1
$$

Observação do lema 3.2: Se tal $A$ existe então, pela proposição 2.2, podemos achar um operador $A^{\prime}$ tal que o símbolo total de $B=A A^{\prime}$ é identicamente um próximo de $\left(x_{0}, \xi_{0}\right)$ e, além disso, se $\left(x_{0}, \xi_{0}\right) \notin W F u$ existe um operador pseudodiferencial $B$ de ordem zero com $B u \in C^{\infty}$ e seu símbolo total é 1 numa vizinhança de $\left(x_{0}, \xi_{0}\right)$.

Lema 3.4 Seja $\pi: X \times\left(\mathbb{R}^{n} \backslash\{0\}\right) \rightarrow X$ a projeção natural e $u \in \mathcal{D}^{\prime}(X)$. Então

$$
\pi W F(u)=\operatorname{suppsing}(u)
$$

Prova: $\Rightarrow$ Se $x_{0} \notin$ suppsing $(u)$ escolhemos $\phi(x) \in C_{0}^{\infty}(X)$ tal que $\phi=1$ numa vizinhança de $x_{0}$ e $\phi=0$ numa vizinhança do suporte singular de $u$. Então vemos que $\phi u \in C_{0}^{\infty}(X)$ portanto $\phi u \in \mathcal{S}\left(\mathbb{R}^{n}\right)$, isto é, $x_{0} \notin \pi W F(u)$.

$\Leftarrow$ Seja $x_{0} \notin \pi W F(u)$. Então para qualquer $\xi_{0} \in \mathbb{R}^{n} \backslash\{0\}$ existe uma função $\phi_{\xi_{0}}(x) \in C_{0}^{\infty}(X)$ e uma vizinhança cônica $\Gamma_{\xi_{0}}$ de $\xi_{0}$ tal que $\phi_{\xi_{0}}(x)=1$ perto de $x_{0}$ e $\widehat{\phi_{\xi_{0}}} u(\xi)$ decresce rapidamente em $\Gamma_{\xi_{0}}$. Seja $\Gamma_{\xi_{1}}, \Gamma_{\xi_{2}}, \ldots . \Gamma_{\xi_{N}}$ um cobrimento de $\mathbb{R}^{n} \backslash\{0\}$. Tomando $\phi=\prod_{j=1}^{N} \phi_{\xi}$, temos que $\widehat{\phi u}(\xi)$ decresce rapidamente em toda parte. Logo $\phi u \in C_{0}^{\infty}(X)$, isto é, $u \in C^{\infty}$ numa vizinhança de $x_{0}, \quad x_{0} \notin$ $\operatorname{suppsing}(u)$. 
O conjunto $W F(u)$ é um refinamento da noção de suporte singular. Em verdade, podemos construir distribuições com conjunto frente de onda arbitrário conforme nos mostra o seguinte teorema:

Teorema 3.1 Se $X$ é um conjunto aberto em $\mathbb{R}^{n}$ e $S$ um subconjunto cônico fechado de $X \times\left(\mathbb{R}^{n} \backslash\{0\}\right)$ então podemos encontrar $u \in \mathcal{D}^{\prime}(X)$ com $W F(u)=S$.

Prova: O elemento principal da prova consiste em um argumento micro-local conhecido como condensação de singularidades. É suficiente provar o fato quando $X=\mathbb{R}^{n}$. Para o outro caso aplicamos o mesmo argumento para o fecho de $S$ em $\mathbb{R}^{n} \times\left(\mathbb{R}^{n} \backslash\{0\}\right)$.

Escolhamos uma sequência $\left(x_{k}, \theta_{k}\right) \in S, \operatorname{com}\left|\theta_{k}\right|=1$, tal que todo $(x, \theta) \in S$ com $|\theta|=1$ é o limite de uma subsequência. Seja $\phi \in C_{0}^{\infty}$ e $\hat{\phi}(0)=1$. Então

$$
u(x)=\sum_{1}^{\infty} k^{-2} \phi\left(k\left(x-x_{k}\right)\right) e^{i k^{3}<x, \theta_{k}>}
$$

é uma função contínua em $\mathbb{R}^{n}$, e vamos provar que $W F(u)=S$. Provemos que $W F(u) \subset S$. Se $\left(x_{0}, \xi_{0}\right) \notin S$ podemos escolher uma vizinhança aberta $U$ de $x_{0}$ e uma vizinhança cônica aberta $V$ de $\xi_{0}$ tal que

$$
(U \times V) \cap S=\emptyset
$$

escrevemos $u=u_{1}+u_{2}$ onde $u_{1}$ é a soma dos termos em (3.5) com $x_{k} \notin U$ e $u_{2}$ é a soma dos termos em (3.5) com $x_{k} \in U$. Então $u_{1} \in C^{\infty}$ numa vizinhança aberta $U_{1}$ de $x_{0}$, com efeito sejam $B_{k}\left(x_{k}, 1 / k\right)$ vizinhança aberta de $x_{k}$ e $C=\operatorname{dist}\left(U_{1}, S\right)$, se $\operatorname{supp} B_{k} \cap U_{1} \neq \emptyset$ então $\frac{1}{k}>C, \operatorname{logo} k<\frac{1}{C}$, isto é, todos exceto um número finito de termos são zero em $U_{1}$ se $\overline{U_{1}} \subset U$. Agora

$$
\widehat{u_{2}}(\xi)=\sum_{x_{k} \in U} k^{-2-n} \hat{\phi}\left(\left(\xi-k^{3} \theta_{k}\right) / k\right) e^{i<x_{K}, k^{3} \theta_{k}-\xi>} .
$$

Por (3.6) vemos que $\theta_{k} \notin V$. Se $V_{1}$ é outra vizinhança cônica de $\xi_{0}$ e $\overline{V_{1}} \subset V \cup\{0\}$, então $|\xi-\eta| \geq C(|\xi|+|\eta|)$ quando $\xi \in V_{1}$ e $\eta \notin V$, para algum $C>0$, dado que é verdade quando $|\xi|+|\eta|=1$. Logo se $|\xi|+|\eta|>M$ temos que $\left|\frac{\xi}{M}\right|+\left|\frac{\eta}{M}\right|=1$ e $\left|\frac{\xi}{M}-\frac{\eta}{M}\right| \geq C\left|\frac{\xi}{M}\right|+\left|\frac{\eta}{M}\right|$. Portanto $|\xi-\eta| \geq C M=C(|\xi|+|\eta|)$. Assim 
$\left|\xi-k^{3} \theta_{k}\right| \geq C\left(|\xi|+k^{3}\right) \geq C|\xi|^{2 / 3} k, \quad \xi \in V_{1}$, e como $\hat{\phi} \in \mathcal{S}$ então $\widehat{u_{2}}$ é de decrescimento rápido em $V_{1}$. Portanto $\left(x_{0}, \xi_{0}\right) \notin W F(u)$.

Vamos verificar que $S \subset W F(u)$. Seja $\left(x_{0}, \xi_{0}\right) \in S$. Escolhemos $\chi \in C_{0}^{\infty}$ igual a 1 perto de $x_{0}$. Para provar que $\left(x_{0}, \xi_{0}\right) \in W F(u)$ temos que provar que $\widehat{\chi u}$ não decresce rapidamente numa vizinhança cônica de $\xi_{0}$. Observemos que

$$
\chi(x) \phi\left(k\left(x-x_{k}\right)\right)=\phi_{k}\left(k\left(x-x_{k}\right)\right),
$$

onde $\phi_{k}(x)=\chi\left(\frac{x}{k}+x_{k}\right) \phi(x)$ pertence a um conjunto limitado em $S$.

$$
\widehat{\chi u}(\xi)=\sum k^{-2-n} \widehat{\phi_{k}}\left(\left(\xi-k^{3} \theta_{k}\right) / k\right) e^{i<x_{k}, k^{3} \theta_{k}-\xi>} .
$$

Se $x_{k}$ é próximo de $x_{0}$ e $k$ é grande então $\phi_{k}=\phi$ e por hipótese $\widehat{\phi_{k}}(0)=1$ então, obtemos para $N$ qualquer que

$$
\widehat{\chi u}\left(k^{3} \theta_{k}\right)=\sum_{j \neq k} j^{-2-n} \widehat{\phi_{k}}\left(\left(k^{3} \theta_{k}-j^{3} \theta_{j}\right) / j\right) e^{i<x_{j}, j^{3} \theta_{j}-k^{3} \theta_{k}>}+k^{-2-n} .
$$

Assim,

$$
\begin{aligned}
\left|k^{-2-n}\right| & \leq\left|\widehat{\chi u}\left(k^{3} \theta_{k}\right)\right|+\sum_{j \neq k} j^{-2-n}\left|\widehat{\phi_{k}}\left(\left(k^{3} \theta_{k}-j^{3} \theta_{j}\right) / j\right)\right| \\
& \leq\left|\widehat{\chi u}\left(k^{3} \theta_{k}\right)\right|+\sum_{j \neq k} j^{-2-n} \mid C_{N}\left(1+\left|\frac{\left(k^{3} \theta_{k}-j^{3} \theta_{j}\right)}{j}\right|\right)^{-N} \\
& \leq\left|\widehat{\chi u}\left(k^{3} \theta_{k}\right)\right|+\sum_{j \neq k} j^{-2-n} C_{N}\left(\left|\frac{\left(k^{3} \theta_{k}-j^{3} \theta_{j}\right)}{j}\right|\right)^{-N}
\end{aligned}
$$

Portanto,

$$
\left|\widehat{\chi u}\left(k^{3} \theta_{k}\right)\right| \geq k^{-2-n}-\sum_{j \neq k} j^{-2-n} C_{N}\left(\left|\frac{\left(k^{3} \theta_{k}-j^{3} \theta_{j}\right)}{j}\right|\right)^{-N} .
$$

Como

$$
\left|k^{3} \theta_{k}-j^{3} \theta_{j}\right| \geq\left|k^{3}-j^{3}\right| \geq k^{2}+k j+j^{2} \geq k j \quad \text { se } k \neq j
$$

temos

$$
\sum_{j \neq k} j^{-2-n} C_{N}\left(\left|\frac{\left(k^{3} \theta_{k}-j^{3} \theta_{j}\right)}{j}\right|\right)^{-N} \leq \sum_{j \neq k} j^{-2-n}(k)^{-N},
$$

e assim

$$
\left|\widehat{\chi u}\left(k^{3} \theta_{k}\right)\right| \geq k^{-2-n}-C_{N} k^{-N} \sum_{j \neq k} j^{-2-n} .
$$


A soma que aparece no lado direito é $O\left(k^{-N}\right)$. Se escolhermos $N>n+2$ obtemos, para $k$ grande,

$$
\left|\widehat{\chi u}\left(k^{3} \theta_{k}\right)\right| \geq \frac{k^{-2-n}}{2},
$$

se $x_{k}$ está próximo de $x_{0}$. Dado que $\left(x_{0}, \frac{\xi_{0}}{\left|\xi_{0}\right|}\right)$ é um ponto limite da sequência $\left(x_{k}, \theta_{k}\right)$ isto quer dizer que $\widehat{\chi u}$ não pode decrescer rapidamente numa vizinhança próxima de $\xi_{0}$.

Um outro exemplo importante consiste em estudar distribuições que são medidas concentradas em hiperfícies. Um caso particular é:

Teorema 3.2 Sejam $V$ um subespaço linear de $\mathbb{R}^{n}$ e $u=u_{0} d s$, onde $u_{0} \in C^{\infty}(V)$ $e$ ds é a medida n-dimensional euclideana. Então

$$
W F(u)=\operatorname{supp}(u) \times\left(V^{\perp} \backslash\{0\}\right) .
$$

Prova: Se $\chi \in C_{0}^{\infty}$ então

$$
(\widehat{\chi u})(\xi)=\int_{V} e^{-i<x, \xi>} \chi(x) u_{0}(x) d s(x) .
$$

Escrevendo $\xi=\xi^{\prime}+\xi^{\prime \prime}$, onde $\xi^{\prime} \in V$ e $\xi^{\prime \prime} \in V^{\perp}$, vemos que se $\left(x_{0}, \xi_{0}\right) \notin W F(u)$ então $\left(x_{0}, \xi_{0}\right) \notin \operatorname{supp}(u) \times\left(V^{\perp} \backslash\{0\}\right)$. Se $\left(x_{0}, \xi_{0}\right) \notin W F(u)$ então $(\widehat{\chi u})(\xi)$ é uma função de decrescimento rápido de $\xi^{\prime}$, pois $\chi u \in C_{0}^{\infty}(V)$ e $(\widehat{\chi u})(\xi)$ não é identicamente nula sobre nenhum conjunto aberto (a menos que $\chi u=0$ ), e $W F(u) \subset \operatorname{supp}(u) \times\left(V^{\perp} \backslash\{0\}\right)$ já que $\widehat{\chi u}$ não decresce rapidamente em nenhum cone aberto contendo $V^{\perp}$ (a menos que $\left.\chi u=0\right)$, mas é rapidamente decrescente em todo cone onde $|\xi| \leq c\left|\xi^{\prime}\right|$.

Teorema 3.3 Seja uma faixa complexa de largura $\delta>0$ sobre um intervalo real $I=[a, b], U=\{z=x+i y, \Re z \in[a, b],|\Im z|<\delta\}$ e $f \in \mathcal{O}(U)$ uma função holomorfa satisfazendo

como no teorema 1.1. Então

$$
|f(z)| \leq \frac{C}{|\Im z|^{N}}, \quad z \in U
$$

$$
W F(f(x \pm i 0)) \subset\{(x, \xi): x \in I \quad \text { e } \quad \xi \gtrless 0\} .
$$


Prova: Se $\phi \in C_{0}^{\infty}(I)$, na segunda prova do teorema 1.1 (valor de fronteira) cumpre-se (1.3) $\operatorname{com} \nu \geq N$. Assim,

$$
\begin{aligned}
(\phi \hat{b}(f))(\xi) & =<b(f) e^{-i<., \xi>}, \phi> \\
& =\int \Phi(x, Y) f(x+i Y) e^{-i<x+i Y, \xi>} d x \\
& +\int_{0}^{\infty} \int_{0}^{1} f(x+i t Y) \phi^{\nu+1}(x) e^{-i<x+i t Y, \xi>}(i Y)^{\nu+1} t^{\nu} / \nu ! d x d t \\
& \leq C_{\phi, \nu}\left(e^{Y \cdot \xi}+\int_{0}^{\infty} e^{t Y \cdot \xi} t^{\nu} d t\right) \\
& \leq C_{\phi, \nu}\left(e^{Y . \xi}+\nu !(-i Y)^{-(\nu+1)}\right) .
\end{aligned}
$$

O lado direito é $O\left(|\xi|^{-(\nu+1)}\right)$ quando $\xi<0$.

Exemplo: Seja $f(z)=\frac{1}{z}$. Então, pelo teorema $3.3 W F\left(f_{0}\right)=\{(0, \xi) / \xi>0\}$, isto é, $W F\left(P\left(\frac{1}{x}\right)-i \pi \delta(x)\right)=\{(0, \xi) / \xi>0\}$.

$\mathrm{O}$ seguinte teorema é central para descrever as singularidades de uma integral oscilatória.

Teorema 3.4 (Conjunto Frente de onda da integral oscilatória) A integral oscilatória

$$
I_{\phi, a}=\int e^{i \phi(x, \theta)} a(x, \theta) d \theta
$$

definida no teorema 2.3 como uma distribuição, possui conjunto frente de onda contido no conjunto crítico

$$
W F\left(I_{\phi, a}\right) \subset\left\{\left(x, \phi_{x}^{\prime}(x, \theta)\right) ; \quad(x, \theta) \in F \quad e \quad \phi_{\theta}^{\prime}(x, \theta)=0\right\} .
$$

Prova: Seja $\psi \in C_{0}^{\infty}(X)$. Então da definição de $I_{\phi, a}$ significa que

$$
\widehat{\psi I_{\phi, a}(\xi)}=\iint e^{i(\phi(x, \theta)-<x, \xi>)} \psi(x) a(x, \theta) d x d \theta
$$

como uma integral oscilatória. Queremos provar que isto é rapidamente decrescente em qualquer cone fechado $V \subset \mathbb{R}^{n}$ que não intercepta

$$
\left\{\phi_{x}^{\prime}(x, \theta) ; \quad(x, \theta) \in F \quad x \in \operatorname{supp} \psi, \quad \phi_{\theta}^{\prime}(x, \theta)=0\right\} .
$$


Temos então que, para algum $C>0$,

$$
G(\xi, \theta, x)=\left|\xi-\phi_{x}^{\prime}(x, \theta)\right|+|\theta|\left|\phi_{\theta}^{\prime}(x, \theta)\right| \geq C(|\xi|+|\theta|)
$$

se $(x, \theta) \in F, \quad x \in \operatorname{supp} \psi, \quad \xi \in V$. Vamos provar (3.11) quando $|\xi|+|\theta|=1$. Por compacidade só temos que provar que $G(\xi, \theta, x) \neq 0$ quando $(x, \theta) \in F, \quad x \in$ supp $\psi, \quad \xi \in V$. Mostremos por absurdo. Suponhamos que $G(\xi, \theta, x)=0$. Então $\left|\xi-\phi_{x}^{\prime}(x, \theta)\right|=0$ e $|\theta|\left|\phi_{\theta}^{\prime}(x, \theta)\right|=0$. Se $|\theta|=0$ então, como $\phi$ é homogênea de grau 1 com respeito a $\theta$, observamos que $\phi_{x}^{\prime}(x, \theta)$ e $|\theta| \phi_{\theta}^{\prime}(x, \theta)$ são contínuas em $F$, com valor zero quando $\theta=0$ e portanto $G(\xi, \theta, x)=|\xi|=1$, o que contradiz o fato de que $G(\xi, \theta, x)=0$. Se $\left|\phi_{\theta}^{\prime}(x, \theta)\right|=0$ e $\theta \neq 0$, então $\xi \neq \phi_{x}^{\prime}(x, \theta)$, dado que $\xi \in V$, o que contradiz o fato que $\xi=\phi_{x}^{\prime}(x, \theta)$. Logo $G(\xi, \theta, x) \neq 0$. Escolhendo $\chi \in C_{0}^{\infty}\left(\mathbb{R}^{n}\right)$ tal que $\chi(\theta)=1$ quando $|\theta|<1$ e $\chi(\theta)=0$ quando $|\theta|>2$, e colocando $\chi_{\nu}(\theta)=\chi\left(2^{-\nu} \theta\right)-\chi\left(2^{1-\nu} \theta\right) \quad \nu>0, \quad \chi_{0}(\theta)=\chi(\theta)$ então temos $\sum_{0}^{\infty} \chi_{\nu}=1$ e $2^{\nu-1} \leq|\theta| \leq 2^{\nu+1}$ quando $\theta \in \operatorname{supp}_{\nu \neq 0} \chi_{\nu}$. Expressando a integral oscilatória como esta partição da unidade temos

$$
\widehat{\psi I_{\phi, a}(\xi)}=\sum_{0}^{\infty} \iint e^{i(\phi(x, \theta)-<x, \xi>)} \psi(x) \chi_{\nu}(\theta) a(x, \theta) d x d \theta .
$$

Cada termo está em $S$. Com $R=2^{\nu-1}$ os termos com $\nu \neq 0$ podem ser escritos como

$$
R^{N} \iint e^{i(R \phi(x, \theta)-<x, \xi>)} \psi(x) \chi_{1}(\theta) a(x, R \theta) d x d \theta
$$

se $\Phi(x, \theta)=\frac{(R \phi(x, \theta)-\langle x, \xi>)}{R+|\xi|}$ e $\xi \in V$ temos, por (3.11), que

$$
\left|\Phi_{x}^{\prime}\right|+\left|\Phi_{\theta}^{\prime}\right| \geq \frac{C(R|\theta|+|\xi|)}{(R+|\xi|)} \geq C
$$

no suporte de $\psi(x) \chi_{1}(\theta) a(x, R \theta)$. Com $\gamma=\max (1-\rho, \delta)<1$ temos

$$
\left|D_{\theta}^{\alpha} D_{x}^{\beta} \psi(x) \chi_{1}(\theta) a(x, R \theta)\right| \leq C_{\alpha \beta} R^{m+\gamma(|\alpha|+|\beta|)} .
$$

Pelo teorema 2.2 segue que (3.12) é estimado para $k$ grande por

$$
C_{k} R^{m+N+k \gamma}(R+|\xi|)^{-k} \leq C_{k} R^{-1}|\xi|^{m+N+1+k(\gamma-1)}
$$

se $\xi \in V$. Dado que $\sum_{\nu=1}^{\infty} 2^{1-\nu}=2$, concluímos que $\widehat{\psi I_{\phi, a}}(\xi)$ é rapidamente decrescente em $V$. 
Exemplo: O problema de Cauchy para a equação de onda

$$
\partial^{2} f / \partial t^{2}=\Delta f
$$

com

$$
\left.f\right|_{t=0}=0, \quad \partial f /\left.\partial t\right|_{t=0}=u(x)
$$

onde $x \in \mathbb{R}^{n}, f=f(x, t), \Delta$ é o laplaciano em $x$ e $u(x) \in C_{0}^{\infty}\left(\mathbb{R}^{n}\right)$. A solução é a diferença entre as duas integrais oscilatórias com funções fase $(x-y) \xi \pm t|\xi|$

$$
\begin{aligned}
f(t, x) & =(2 \pi)^{-n} \iint e^{i[(x-y) \xi+t|\xi|]}(2 i|\xi|)^{-1} u(y) d y d \xi \\
& -(2 \pi)^{-n} \iint e^{i[(x-y) \xi-t|\xi|]}(2 i|\xi|)^{-1} u(y) d y d \xi .
\end{aligned}
$$

Pelo teorema 3.4 temos que

$$
W F(f) \subset\left\{(x, y, \xi,-\xi):(x-y) \pm \frac{2 t \xi}{|\xi|}=0\right\} .
$$

Sejam $X$ um conjunto aberto em $\mathbb{R}^{n}$ e $\Gamma$ um cone fechado em $X \times\left(\mathbb{R}^{n} \backslash\{0\}\right)$. Definamos

$$
\mathcal{D}_{\Gamma}^{\prime}(X)=\left\{u \in \mathcal{D}^{\prime}(X), W F(u) \subset \Gamma\right\} .
$$

Lema 3.5 A distribuição $u \in \mathcal{D}^{\prime}(X)$ está em $\mathcal{D}_{\Gamma}^{\prime}(X)$ se e somente se para todo $\phi \in C_{0}^{\infty}(X)$ e para todo cone fechado $V \subset \mathbb{R}^{n}$ com

$$
\Gamma \cap(\operatorname{supp} \phi \times V)=\emptyset
$$

temos

$$
\sup _{V}|\xi|^{N}|\widehat{\phi u}(\xi)|<\infty, \quad N=1,2 \ldots
$$

Prova: $\Leftarrow(3.16)$ implica que $(x, \xi) \notin W F(u)$ se $\phi(x) \neq 0$ e $\xi \in V$. Logo $W F(u) \subset \Gamma$.

$\Rightarrow$ Se $u \in \mathcal{D}_{\Gamma}^{\prime}(X)$ e $\xi \in \phi u$ então $(x, \xi) \in \Gamma$ para algum $x \in$ supp $\phi$, lema 3.1. Também $\xi \notin V$ por (3.15). Logo $\sup _{V}|\xi|^{N}|\widehat{\phi u}(\xi)|<\infty, \quad N=1,2 \ldots$ 
Teorema 3.5 Sejam $u, v \in \mathcal{D}^{\prime}(X)$ e suponha que $(x, \xi) \in W F(u) \Rightarrow(x,-\xi) \notin$ $W F(v)$. Então o produto uv é bem definido $e$

$W F(u v) \subset\{(x . \xi+\eta):(x, \xi) \in W F(u), \quad(x, \eta) \in W F(v)\} \cup W F(u) \cup W F(v)$.

Prova: Usando uma partição da unidade $C^{\infty}$ que consiste em funções com suporte pequeno. podemos assumir que $u, v \in \mathcal{E}^{\prime}(X)$ e que além disso

$$
\xi \in \Gamma(u) \Rightarrow-\xi \notin \Gamma(v)
$$

Então segue que

$$
\left.(\hat{u} * \hat{v})(\xi)=\int \hat{u}(\xi-\eta) \hat{v}\right)(\eta) d \eta
$$

é uma integral absolutamente convergente. Se $\eta$ pertence a uma vizinhança cônica pequena de $\Gamma(v)$ então, para $\xi$ fixo, $\hat{u}(\xi-\eta)=O\left(|\eta|^{-N}\right)$, enquanto que se $\eta$ está fora desta vizinhança cônica pequena de $\Gamma(v), \hat{v}(\eta)=O\left(|\eta|^{-N}\right)$ por definição. Assim, (3.19) converge absolutamente e é temperada, já que $|\hat{u}(\xi)|, \quad \hat{v}(\xi) \mid \leq$ $C(1+|\xi|)^{m}$ para algum $m$. Assim, $u v$ é bem definida e pertence a $\mathcal{E}^{\prime}(X)$ pela fórmula inversa de Fourier. Para provar (3.17) escrevemos $\hat{u}=\hat{u}_{0}+\hat{u_{1}} \mathrm{e} \cdot \hat{v}=$ $\hat{v}_{0}+\hat{v}_{1}$, onde $\hat{u}_{0}, \hat{v_{0}} \in S$ e $\hat{u_{1}}, \hat{v_{1}}$ é identicamente nula fora de uma vizinhança cônica pequena de $\Gamma(u)$ e $\Gamma(v)$, respectivamente. Então, segue que a transformada de Fourier de uv é

$$
\left.(2 \pi)^{-n} \sum_{j, k=0,1} \int \hat{u}_{j}(\xi-\eta) \hat{v}_{k}\right)(\eta) d \eta
$$

Se $j$ e $k$ são ambos nulos, então esta convolução é rapidamente decrescente em todas as direções. Se $j=0$ e $k=1$ ou $j=1$ e $k=0$, segue da prova do lema 3.1 que (3.20) é rapidamente decrescente fora da vizinhança cônica pequena de $\Gamma(v)$ ou $\Gamma(u)$, respectivamente. Além disso, por construção, o termo restante onde $j=k=1$ é identicamente nulo para $\xi$ fora da vizinhança cônica pequena de $\Gamma(u)+\Gamma(v)$. to mesmo tempo, essas observações levam a

$$
\Gamma(u v) \subset \Gamma(u) \cup \Gamma(v) \cup \Gamma(u)+\Gamma(v)
$$

o que implica (3.17). 
No teorema seguinte vamos mostrar a fórmula de mudança de variáveis para o conjunto frente de ondas. Assumimos que

$$
f: X \mapsto Y
$$

é um difeomorfismo entre conjuntos abertos de $\mathbb{R}^{n}$. Se $\Lambda$ é um subconjunto cônico fechado de $Y \times \mathbb{R}^{n} \backslash\{0\}$ definimos o pullback de $\Lambda f^{*}: \mathcal{D}_{\Lambda}^{\prime}(Y) \rightarrow \mathcal{D}_{f^{*} \Lambda}^{\prime}(X)$ por

$$
f^{*} \Lambda=\left\{(x, \xi):\left(f(x),\left({ }^{\prime} f^{\prime}(x)\right)^{-1} \xi\right) \in \Lambda\right\} .
$$

Teorema 3.6 Sejam $X$ e $Y$ conjuntos abertos de $\mathbb{R}^{n}$ e $f: X \mapsto Y$ um difeomorfismo. Então $W F\left(f^{*} u\right)=f^{*} W F(u)$.

Prova: Já que o problema é local, não existe perda de generalidade se assumirmos que $u \in \mathcal{E}^{\prime}$. Como $\left(f^{-1}\right)^{*} f^{*}=$ identidade, basta provar que $W F\left(f^{*} u\right) \subset$ $f^{*} W F(u)$, pois a outra inclusão é uma consequência imediata. De fato,

$$
f^{*}(W F(u))=f^{*}\left(W F\left(f^{-1}\right)^{*}\left(f^{*} u\right)\right) \subset f^{*}\left[\left(f^{-1}\right)^{*} W F\left(f^{*} u\right)\right]=W F\left(f^{*} u\right) .
$$

Podemos assumir que $0 \in X, f(0)=0$ e $f^{\prime}(0)=$ identidade. Então, se $\Gamma_{1}$ é uma vizinhança cônica pequena de $\Gamma(u)$, vamos provar que

$$
\Gamma\left(f^{*}(\phi u)\right) \subset \Gamma_{1},
$$

se $\phi \in C_{0}^{\infty}\left(\mathbb{R}^{n}\right)$ é suportada numa vizinhança pequena da origem. Isto implica que $W F\left(f^{*} u\right) \subset f^{*} W F(u)$. Para provar isto, fixamos $\beta \in C_{0}^{\infty}\left(\left\{\xi: \frac{1}{2}<\xi<2\right\}\right)$ tal que $\sum \beta\left(2^{-j} \xi\right)=1, \xi \neq 0$, e $\beta_{j}(\xi)=\beta\left(2^{-j} \xi\right)$ para $j=1,2 \ldots$ e $\beta_{0}(\xi)=$ $1-\sum_{1}^{\infty} \beta_{j}(\xi)$. Então, se $\rho=f^{*} \phi$,

$$
f^{*}(\phi u)(x)=(2 \pi)^{-n} \sum_{j=0}^{\infty} \rho(x) \int e^{i<f(x), \eta>} \hat{u}(\eta) \beta_{j}(\eta) d \eta .
$$

Podemos escrever $\hat{u}=\hat{u}_{0}+\hat{u}_{1}$, onde $\hat{u}_{0} \in \mathcal{S}$ e supp $\hat{u}_{1} \subset \Gamma_{0}$, onde $\Gamma_{0}$ é um subconjunto fechado de $\Gamma_{1}$ tal que $\Gamma(u) \subset \Gamma_{0} \subset \Gamma_{1}$. Como a transformada de Fourier

$$
(2 \pi)^{-n} \sum_{j=0}^{\infty} \iint \rho(x) e^{-i<x, \xi\rangle} e^{i<f(x), \eta\rangle} \hat{u}_{0}(\eta) \beta_{j}(\eta) d x d \eta
$$


é rapidamente decrescente em todas as direções, basta provar que $\left|\iint e^{-i<x, \xi>} e^{i<f(x), \eta>} \rho(x) \hat{u}_{1}(\eta) \beta_{j}(\eta) d x d \eta\right| \leq C_{N}\left(2^{j}+\mid \xi\right)^{-N}, \quad \xi \notin \Gamma_{1}, j=1,2 \ldots$

Para provar isto tomamos $R=2^{j}$ e escrevemos cada termo como

$$
\begin{aligned}
& R^{n} \iint e^{i[R<f(x), \eta>-<x, \xi>]} \rho(x) \hat{u}_{1}(R \eta) \beta_{1}(\eta) d x d \eta \\
& =R^{n} \iint e^{i[R+|\xi| \Phi(X, \xi, \eta)]} \rho(x) \hat{u}_{1}(R \eta) \beta_{1}(\eta) d x d \eta,
\end{aligned}
$$

$\operatorname{com} \Phi=[R<f(x), \eta>-<x, \xi>] /(R+|\xi|)$. Notemos que o integrando tem suporte compacto fixo. Além disso, como assumimos que $f^{\prime}(0)=$ identidade, isto implica que $f(x)=x+O\left(|x|^{2}\right)$. Daí, se $\rho$ é suportada numa vizinhança pequena de zero então, se $\xi \notin \Gamma_{1}$ temos que $|\xi-\eta| \geq(|\xi|+|\eta|)$ e portanto

$$
\begin{aligned}
\left|\nabla_{x} \Phi\right| & =\frac{|R \eta-\xi+O(|x| R \eta)|}{R+|\xi|} \\
& \leq C_{0} \frac{|R \eta|+|\xi|}{R+|\xi|}-O(|x|) \leq C,
\end{aligned}
$$

para algum $C>0$ sobre o suporte de $\rho(x) \hat{u_{1}}(R \eta) \beta_{1}(\eta)$.

Dado que $\left|\hat{u}_{1}(R \eta)\right| \leq C R^{m}$, para algum $m$, o resultado é consequência do lema 2.2.

Exemplo: Para as distribuições $(A \pm i 0)^{(2-n) / 2}$ em $\mathbb{R}^{n+1}$ temos

$$
W F\left((A \pm i 0)^{(2-n) / 2}\right)=\{(x, t d A(x)), x \neq 0, A(x)=0, t \gtrless 0\} \cup T_{0}^{*} \backslash\{0\} .
$$

De fato, analogamente ao exemplo anterior, temos que

$$
W F\left((t \pm i 0)^{(2-n) / 2}\right) \subset\{(0, \tau), \quad \tau \gtrless 0\}
$$

e sabemos que $(A \pm i 0)^{(2-n) / 2}=A^{*}\left((t \pm i 0)^{(2-n) / 2}\right)$. Então, pelo teorema 3.6

$$
W F\left[A^{*}\left((t \pm i 0)^{(2-n) / 2}\right)\right] \subset A^{*}\left[W F\left((t \pm i 0)^{(2-n) / 2}\right)\right]=A^{*}\{(0, \tau), \quad \tau \gtrless 0\}
$$

e portanto,

$W F\left((A \pm i 0)^{(2-n) / 2}\right) \subset\{(x, t d A(x)), x \neq 0, A(x)=0, t \gtrless 0\} \quad$ quando $\quad x \neq 0$. 
Pelo teorema 1.4 temos que

$$
P(\partial)(A \pm i 0)^{(2-n) / 2}=C \delta_{0}
$$

Assim, pelo exemplo (1.), sabemos que $W F\left(\delta_{0}\right)=T_{0}^{*} \backslash\{0\}$ e, como $W F(P u) \subset$ $W F(u)$, temos

$$
W F\left(B(\partial)(A \pm i 0)^{(2-n) / 2}\right) \subset W F\left((A \pm i 0)^{(2-n) / 2}\right),
$$

e, portanto, $T_{0}^{*} \backslash\{0\}=W F\left(\delta_{0}\right) \subset W F\left((A \pm i 0)^{(2-n) / 2}\right)$. Como $(A \pm i 0)^{(2-n) / 2}$ não é $C^{\infty}$ em qualquer $x \neq 0$ onde $A(x)=0$, vem que

$$
W F\left((A \pm i 0)^{(2-n) / 2}\right)=\{(x, t d A(x)), x \neq 0, A(x)=0, t \gtrless 0\} \cup T_{0}^{*} \backslash\{0\} .
$$

Na prova do teorema 1.4 temos que $\chi_{ \pm}^{a}$ pode escrever-se como uma combinação linear de $(t \pm i 0)^{a}$ quando $a \notin \mathbb{R}_{+}$, então segue que em $\mathbb{R}^{n} \backslash\{0\}$

$$
W F\left(A^{*} \chi_{ \pm}^{(1-n) / 2}\right)=\{(x, t d A(x)), x \neq 0, A(x)=0, t \neq 0\}
$$




\section{Capítulo 4}

\section{Operadores integrais de Fourier}

\section{1 Álgebra de simplectomorfismos}

Definição 4.1 Uma forma bilinear $w: V \times V \rightarrow \mathbb{R}^{n}$ num espaço vetorial $V$ é chamada simplética se

$$
w(x, y)=-w(y, x) \quad \forall x, y \in V
$$

e w é não degenerada, isto é, se $x \in V$ é tal que $w(x, y)=0 \quad \forall y \in V$ implicar que $x=0$. Neste caso o par $(V, w)$ é dito ser um espaço vetorial simplético.

Note que (4.1) é equivalente a $\forall x \in V, w(x, x)=0$, ainda mais, que a $\operatorname{dim} V$ é um número par.

Exemplo: Seja o espaço vetorial $\mathbb{R}^{n}$. Usando a base canônica $\left(e_{1}, \ldots e_{2 n}\right)$ de $\mathbb{R}^{2 n}$, podemos identificar $\mathbb{R}^{n}$ com o seu dual. Denotemos por $x^{1}, \ldots x^{n}, p^{1}, \ldots p^{n}$ as coordenadas padrão em $\mathbb{R}^{n} \times \mathbb{R}^{n}$, e por $(x, p)$ o ponto variável ali. Temos

$$
\begin{aligned}
w_{n}\left((x, p),\left(x^{\prime}, p^{\prime}\right)\right) & =\sum_{j=1}^{n} x_{j} p_{j}^{\prime}-p_{j} x_{j}^{\prime} \\
& =\left(\begin{array}{ll}
x & p
\end{array}\right)^{t}\left(\begin{array}{cc}
0 & I_{n} \\
-I_{n} & 0
\end{array}\right)\left(\begin{array}{c}
x^{\prime} \\
p^{\prime}
\end{array}\right) .
\end{aligned}
$$

Então $\left(\mathbb{R}^{2 n}, w_{n}\right)$ é um espaço simplético. 
Definição 4.2 Consideremos uma forma simplética $\sigma$ sobre $T^{*}\left(\mathbb{R}^{n}\right)^{\prime}=\mathbb{R}^{2 n}$. Dizemos que os vetores unitários linearmente independentes $\left\{e_{j}\right\}_{j=1}^{n}, \quad\left\{\epsilon_{j}\right\}_{j=1}^{n}$ formam uma base simplética se

$$
\sigma\left(e_{j}, e_{k}\right)=\sigma\left(\epsilon_{j}, \epsilon_{k}\right)=0, \quad \sigma\left(\epsilon_{j}, e_{k}\right)=-\sigma\left(e_{j}, \epsilon_{k}\right)=\delta_{j k} \quad \forall j, k=1, \ldots, n .
$$

Isto é, nesta base a matriz $\sigma$ toma a forma:

$$
\sigma=\left(\begin{array}{cc}
0 & I_{n} \\
-I_{n} & 0
\end{array}\right) .
$$

Teorema 4.1 Se $\left\{e_{j}\right\}_{j=1}^{n_{1}}, \quad\left\{\epsilon_{j}\right\}_{j=1}^{n_{2}}$ são vetores linearmente independentes em $\mathbb{R}^{n}$ que cumprem (4.2) para quaisquer $1 \leq j \leq n_{1}, 1 \leq j \leq n_{2}$, então podemos escolher vetores unitários adicionais tais que $e_{1}, \ldots e_{n}, \epsilon_{1}, \ldots, \epsilon_{n}$ formam uma base simplética de $\mathbb{R}^{2 n}$.

Definição 4.3 Seja $W \subset V$ um subespaço linear do espaço simplético $(V, w)$. O complemento ortogonal simplético de $W$, denotado $W^{\perp}$, é definido como

$$
W^{\perp}=\{x \in V, \quad \forall y \in W, \quad w(x, y)=0\} .
$$

Notemos que $W^{\perp} \cap W$ contém vetores não nulos, quando $\operatorname{dim} W=1$. Note também que $W^{\perp}=W$ implica que a $\operatorname{dim} W=n$. Isto segue do fato que $w$ é não degenerada.

Definição $4.4 O$ subespaço vetorial $W \subset V$ é chamado

1. isotrópico se $W \subset W^{\perp}$;

2. coisotrópico ou (involutivo) se $W^{\perp} \subset W$;

3. lagrangeano se $W^{\perp}=W$;

4. simplético se $W^{\perp} \cap W=\{0\}$.

Exemplo: Denotemos por $\left(e_{1}, \ldots e_{n}, \epsilon_{1}, \ldots, \epsilon_{n}\right)$ a base canônica em $\mathbb{R}^{2 n}$. Se usarmos isto para identificar $\mathbb{R}^{2 n}$ como seu próprio dual, e então consideramos $w_{n}$ como um endomorfismo de $\mathbb{R}^{2 n}$, vemos que isto é representado pela matriz

$$
\mathcal{T}_{\ltimes}=\left(\begin{array}{cc}
0 & I_{n} \\
-I_{n} & 0
\end{array}\right),
$$


onde os blocos são matrizes $n \times n\left(I_{n}\right.$ : matriz identidade $\left.n \times n\right)$. Temos

$$
\mathcal{T}_{n}^{2}=-I_{2 n}^{2},
$$

implicando em $\mathcal{T}_{n}^{-1}=\mathcal{T}_{n}^{t}=-\mathcal{T}_{n}$, e assim, o endomorfismo associado com a matriz $\mathcal{T}_{n}$ é uma estrutura complexa sobre $\mathbb{R}^{2 n}$, a qual identifica $\mathbb{R}^{2 n}$ com $\mathbb{C}^{n}$. Temos, para algum $z, z^{\prime}$ em $\mathbb{R}^{n}$,

$$
w_{n}\left(z, z^{\prime}\right)=\left(\mathcal{T}_{n} z\right) \cdot z^{\prime} .
$$

A matriz $\mathcal{T}_{n}$ é chamada a matriz fundamental da estrutura simplética em $\left(\mathbb{R}^{2 n}, w_{n}\right)$. Se $W=\left\{e_{1}\right\}$ então $W^{\perp}=\left\{e_{1}, \ldots e_{n}, \epsilon_{2}, \ldots, \epsilon_{n}\right\}$ e portanto $W \subset W^{\perp}$. Assim $W$ é isotrópico.

Se $W=\left\{e_{1}, \ldots e_{n}, \epsilon_{1}\right\}$ então $W^{\perp}=\left\{e_{2}, \ldots e_{n}\right\}$ e portanto $W^{\perp} \subset W$. Assim $W$ é coisotrópico.

Se $W=\left\{e_{1}, \ldots e_{n}\right\}$ então $W^{\perp}=\left\{e_{1}, \ldots e_{n}\right\}$ e portanto $W=W^{\perp}$. Assim $W$ é lagrangeano.

Definição 4.5 Seja w uma 2-forma $C^{\infty}$ sob uma variedade $X$ tal que, para todo $x \in X, w_{x}$ define um funcional bilinear simplético sob $T_{x} X$. Suponhamos que $w$ é fechada. O par $(X, w)$ é chamado uma variedade simplética.

\section{Exemplo:}

Seja $M$ uma variedade $C^{\infty}$ arbitrária, de dimensão $n$. Então o fibrado cotangente $T^{*} M$ pode ser equipado com uma estrutura de variedade simplética, por meio da forma simplética fundamental $w$. Sejam $\left(x^{1}, \ldots x^{n}\right)$ coordenadas locais em $M$. Denotamos por $\left(\xi_{1}, \ldots \xi_{n}\right)$ as coordenadas associadas no espaço cotangente. A expressão de $w$ nas coordenadas locais $\left(x^{1}, \ldots x^{n}, \xi_{1}, \ldots \xi_{n}\right)$ em $T^{*} M$ é

$$
w=\sum_{j=1}^{n} d \xi_{j} \wedge d x_{j} .
$$

Isto significa que se temos dois campos vetoriais $\theta_{j} \in C^{\infty} \quad j=1,2$ sob $O \subset T^{*} M$ aberto, cujas expressões, em alguma carta local em $O$ levantada de alguma carta local $\left(U, x^{1}, \ldots x^{n}\right)$ na base de $M$, são

$$
\theta_{j}=\sum_{k=1}^{n} \alpha_{j}^{k}(x, \xi) \frac{\partial}{\partial x_{k}}+\beta_{j k}(x, \xi) \frac{\partial}{\partial \xi_{k}},
$$


então

$$
w\left(\theta_{1}, \theta_{2}\right)=\sum_{k=1}^{n}\left(\alpha_{2}^{k} \beta_{1 k}-\alpha_{1}^{k} \beta_{2 k}\right)(x, \xi) .
$$

A forma $w$ é fechada, porque $w$ é a derivada exterior da 1-forma simplética fundamental $\xi d x$.

Definição 4.6 Sejam L, M subespaços lagrangianos de $V$ cuja intersecção é somente a origem. Dizemos que $L$ e $M$ são transversos se

$$
V=L \oplus M \quad(\oplus: \text { soma direta })
$$

isto é, se $\operatorname{dim} V=\operatorname{dim} L+\operatorname{dim} M$.

Observação: Sejam $X$ uma variedade $C^{\infty}$ e $Y, Z \subset X$ subvariedades $C^{\infty}$. Dizemos que $Y, Z$ intercectam-se transversalmente em $x_{0} \in Y \cap Z$ se

$$
T_{x_{0}} X=T_{x_{0}} Y+T_{x_{0}} Z
$$

Vamos enunciar agora um lema que será útil mas adiante

Lema 4.1 Se $V_{0}$ e $V_{1}$ são dois subespaços lagrangeanos de $T^{*}\left(\mathbb{R}^{n}\right)$ podemos achar um terceiro subespaço lagrangeano $V$ o qual é transverso a ambos $V_{0}$ e $V_{1}$.

Sua prova será apresentada no apêndice.

Definição 4.7 Uma subvariedade $C^{\infty}$ (imersão) $\Lambda$ de $T^{*} X \backslash\{0\}$ é chamada uma subvariedade lagrangeana, se dado $(x, \xi) \in \Lambda$ temos que $W^{\perp}=W$, onde $W=T_{x, \xi} \Lambda$ é o espaço tangente de $\Lambda \mathrm{em}(x, \xi)$.

Definição 4.8 Uma função $\phi \in C^{\infty}\left(X \times \mathbb{R}^{n}\right)$ é chamada uma função de fase não degenerada (homogênea) se é uma função de fase e além disso se o posto $\left(\frac{\partial^{2} \phi}{\partial x \partial \theta}\right)=n$, isto é, se os diferencias $d\left(\frac{\partial \phi}{\partial \theta_{j}}\right), j=1, \ldots, N$ são linearmente independentes.

Definimos o conjunto cônico

$$
\Sigma_{\phi}=\left\{(x, \theta) \in \Gamma: \phi_{\theta}^{\prime}(x, \theta)=0\right\} .
$$


Notemos que a hipótese extra implica que $\Sigma_{\phi}$ é uma subvariedade de $\Gamma$ n-dimensional $C^{\infty}$. Além disso a aplicação

$$
\Sigma_{\phi} \ni(x, \theta) \mapsto\left(x, \phi_{x}^{\prime}(x, \theta)\right) \in T^{*} X \backslash\{0\}
$$

é um difeomorfismo. O posto de (4.3) é $n$ em todo ponto de $\Sigma_{\phi}$. Assim,

$$
\Lambda_{\phi}=\left\{\left(x, \phi_{x}^{\prime}(x, \theta)\right):(x, \theta) \in \Gamma \quad \text { e } \quad \phi_{\theta}^{\prime}(x, \theta)=0\right\}
$$

é uma subvariedade suave n-dimensional (imersão) de $T^{*} X \backslash\{0\}$.

Vamos examinar uma classe importante de subvariedades lagrangeanas associadas a um FIO.

Teorema 4.2 Se 申é uma função fase não degenerada então $\Lambda_{\phi}$ é uma subvariedade lagrangeana de $T^{*} X \backslash\{0\}$.

Prova: Dado $v=\xi . d x=\sum_{j=1}^{n} \xi_{j} d x_{j}$ tal que $d v=\rho$. Podemos ver que $v$ restrita a $\Lambda_{\phi}$ é identicamente nula. O pullback de $v$ a $\Sigma_{\phi}$ pelo mapeamento $k$ em (4.3) é

$$
\phi_{x}^{\prime} d x=d \phi-\phi_{\theta}^{\prime} d \theta .
$$

Isto é identicamente nulo sob $\Sigma_{\phi}$, dado que, pelas relações de homogeneidade de Euler, $\phi=\left\langle\phi_{\theta}^{\prime}, \theta\right\rangle$, $\phi$ é identicamente nula sob $\Sigma_{\phi}$. Dado que $k$ é um difeomorfismo, segue que $v$ é identicamente nula sob $\Lambda_{\phi}$.

Definição 4.9 Uma secção $C^{\infty}$ é uma subvariedade da forma $\{(x, \psi(x))\}$ com $\psi(x) \in C^{\infty}$.

Observação 1: Dado que o pullback de $v$ baixo o difeomorfismo $x \mapsto(x, \psi(x))$ é $\psi \cdot d x$ segue do lema de Poincaré que a secção é lagrangeana se e somente se $\psi(x)=\nabla \varphi(x)$ para alguma função $\varphi \in C^{\infty} . \psi \cdot d x$ é fechado quando $\psi$ tem esta forma. Analogamente, temos que, se $H(\xi) \in C^{\infty}\left(\mathbb{R}^{n} \backslash 0\right)$ é real e homogênea de grau 1, então $\Lambda=\left\{\left(H^{\prime}(\xi), \xi\right\}\right.$ é uma subvariedade cônica lagrangeana de $T^{*}\left(\mathbb{R}^{n} \backslash 0\right)$. 
Definição 4.10 Uma subvariedade $C$ de $T^{*}(X \times Y) \backslash\{0\}$ é chamada uma relação canônica homogênea de $T^{*} Y \backslash\{0\}$ a $T^{*} X \backslash\{0\}$ se $C$ é fechada e canônica, se $C$ esta contida em $\left(T^{*} X \backslash\{0\}\right) \times\left(T^{*} Y \backslash\{0\}\right)$ e se $C$ é lagrangeana com respeito à forma simplética $\sigma_{X}-\sigma_{Y}$.

Vamos examinar um exemplo importante de relação canônica.

Exemplo [relação canónica de uma função de fase] Seja $\phi(x, y)$ uma função fase não degenerada então

$$
C_{\phi}=\left\{\left(x, \phi_{x}^{\prime}(x, y), y,-\phi_{y}^{\prime}(x, y)\right)\right\} \subset T^{*}\left(\mathbb{R}^{n}\right) \times T^{*}\left(\mathbb{R}^{n}\right)
$$

é uma relação canônica associada a $\phi$. Pela observação (1) $C_{\phi}$ é lagrangeano com respeito à forma simplética $d \xi \wedge d x-d \eta \wedge d y$. A condição de não degenerada

$$
\operatorname{det}\left(\frac{\partial^{2} \phi}{\partial x_{j} \partial x_{k}}\right) \neq 0
$$

é equivalente à condição de que as duas projeções

$$
C_{\phi}
$$

$$
\left(x, \phi_{x}^{\prime}(x, y)\right) \swarrow \quad \searrow\left(y,-\phi_{y}^{\prime}(x, y)\right)
$$

$\operatorname{com}\left(x, \phi_{x}^{\prime}(x, y)\right)$ e $\left(y,-\phi_{y}^{\prime}(x, y)\right) \in T^{*}\left(\mathbb{R}^{n}\right)$ são difeomorfismos locais, isto é, têm diferenciais sobrejetores.

\subsection{Distribuições Lagrangeanas}

Nesta seção vamos definir o espaço Besov $B_{2}^{\sigma, \infty}\left(\mathbb{R}^{n}\right)$, que será usado na definição de o espaço das distribuições lagrangeanas de ordem $m, \quad I^{m}(X, \Lambda)$, pois na seguinte seção precisamos deste espaço para definir o operador integral de Fourier (FIO), já que seu kernel é um elemento de $I^{m}(X \times Y, \Lambda)$.

Definição 4.11 O espaço Besov $B_{2}^{\sigma, \infty}\left(\mathbb{R}^{n}\right)$ é o espaço de todas as $u \in \mathcal{S}^{\prime}\left(\mathbb{R}^{n}\right)$ para as quais $\hat{u} \in L_{\text {Loc }}^{2}\left(\mathbb{R}^{n}\right)$ e além disso,

$$
\begin{aligned}
\|u\|_{B_{2}^{\sigma, \infty}\left(\mathbb{R}^{n}\right)} & =\left(\int_{|\xi| \leq 1}|\hat{u}(\xi)|^{2} d \xi\right)^{1 / 2} \\
& +\sup _{j \geq 0}\left(\int_{2^{j} \leq|\xi| \leq 2^{j+1}}\left|2^{\sigma_{j}} \hat{u}(\xi)\right|^{2} d \xi\right)^{1 / 2}<\infty .
\end{aligned}
$$


Se $X$ é uma variedade $C^{\infty}$ de dimensão $n$ podemos estender esta definição usando coordenadas locais. Definimos $B_{2}^{\sigma, \infty, L o c}(X)$ como sendo o espaço tal que para todo $u \in \mathcal{D}^{\prime}(X)$ para o qual $(\psi u) \circ k^{-1}$ está em $B_{2}^{\sigma, \infty}\left(\mathbb{R}^{n}\right)$ sempre que $\Omega \subset X$ é uma trajetória coordenada com coordenadas $k$ e $\psi \in C_{0}^{\infty}(\Omega)$.

Definição 4.12 Se $\Lambda \subset T^{*} X \backslash\{0\}$ é uma subvariedade lagrangeana cônica fechada (imersão) $C^{\infty}$, definimos o espaço das distribuições lagrangeanas de ordem $m$ que são associadas a $\Lambda, I^{m}(X, \Lambda)$ como segue. Dizemos que $u \in I^{m}(X, \Lambda)$ se

$$
\prod_{j=1}^{N} P_{j} u \in B_{2}^{-m-n / 4, \infty, L o c}(X),
$$

sempre que $P_{j} \in L^{1}(X)$ são $\Psi D O$ propriamente suportados cujo símbolo principal $p_{j}(x, \xi)$ é identicamente nulo em $\Lambda$.

Teorema 4.3 Se $u \in I_{\text {comp }}^{m}\left(\mathbb{R}^{n}, \Lambda\right)$ com $\Lambda$ da forma $\left\{\left(H^{\prime}(\xi), \xi\right\}\right.$ então temos para $|\xi| \geq 1, \quad \hat{u}(\xi)=e^{-i H(\xi)} v(\xi) \operatorname{com} v \in S^{m-n / 4}\left(\mathbb{R}^{n}\right)$.

Prova: Seja $\rho \in C_{0}^{\infty}$ igual a 1 próximo da origem e seja $\hat{h}=\rho \hat{H}_{0}$ com $H_{0}=$ $(1-\rho) H$. Então $H_{0}-H \in S$ e também isto é suficiente para mostrar que .

$$
v(\xi)=e^{i h(\xi)} \hat{u}(\xi) \in S^{m-n / 4} .
$$

Seja $h_{j}=\frac{\partial h}{\partial \xi_{j}}$. Então, por construção, $h_{j}(D)$ é propriamente suportado dado que este operador é convolução com a transformada inversa de Fourier de $h_{j}(\xi)$ o qual é compactamente suportado. Daí, dado que os símbolos principais de $D_{k}\left(x_{j}-h_{j}(D)\right)$ são identicamente nulos sobre $\Lambda$, de (4.4) temos que

$$
D^{\beta} \prod\left(x_{j}-h_{j}(D)\right)^{\alpha_{j}} u \in B_{2}^{-m-n / 4, \infty} \quad \text { se } \quad|\alpha|=|\beta| .
$$

Isto significa que para $R>1$

$$
\int_{R / 2 \leq|\xi| \leq 2 R}\left|\xi^{\beta} \prod\left(-D_{j}-h_{j}(\xi)\right)^{\alpha_{j}} \hat{u}(\xi)\right|^{2} d \xi \leq C_{\alpha} R^{2(m+n / 4)}, \quad|\alpha|=|\beta|,
$$

ou equivalente

$$
\int_{R / 2 \leq|\xi| \leq 2 R}|\xi|^{2|\alpha|}\left|D^{\alpha} v(\xi)\right|^{2} d \xi \leq C_{\alpha} R^{2(m+n / 4)}, \quad|\alpha|=|\beta|
$$


redefinindo, vemos que $v_{R}(\xi)=v(R \xi) / R^{m-n / 4}$ satisfaz as estimativas uniformes

$$
\int_{1 / 2 \leq|\xi| \leq 2}\left|D^{\alpha} v_{R}(\xi)\right|^{2} d \xi \leq C_{\alpha} \quad \forall \alpha .
$$

Pelo teorema de imersão de Sobolev, isto implica que $\left|D^{\alpha} v_{R}(\xi)\right| \leq C_{\alpha}$ quando $|\xi|=1$, ou equivalentemente, $\left|D^{\alpha} v\right| \leq C_{\alpha}(1+|\xi|)^{-m+n / 4-|\alpha|}$. Na observação (1) vimos que toda secção lagrangiana de $T^{*}\left(\mathbb{R}^{n}\right)$ é localmente o gradiente de uma função $C^{\infty}$.

Teorema 4.4 Seja $\gamma_{0}=\left(x_{0}, \xi_{0}\right) \in \Lambda \subset T^{*} X \backslash\{0\}$, com $\Lambda$ sendo uma variedade lagrangeana cônica $C^{\infty}$. Neste caso, podemos escolher coordenadas locais $(x, \xi)$ : $\Lambda \mapsto \mathbb{R}^{n} \times\left(\mathbb{R}^{n}\right)^{*}$ com as seguintes propriedades

1. $x\left(x_{0}\right)=0$;

2. $(x, \xi) \in \Lambda \mapsto \xi$ é um difeomorfismo local;

3. existe um único $H \in C^{\infty}$ real homogêneo tal que, próximo a $\left(x_{0}, \xi_{0}\right), \Lambda=$ $\left\{\left(H^{\prime}(\xi), \xi\right\}\right.$.

Prova: Primeiro assumimos (1.) e vemos que isto implica (2.). Próximo de $\gamma_{0}$, $\Lambda=\left\{(\phi(\xi), \xi\}\right.$ para algum $\phi$ o qual é homogêneo de grau zero e $C^{\infty}$ próximo de $\xi_{0}$. Na prova do teorema 4.2 dizemos que a 1 -forma canônica $w=\sum \xi_{j} d x_{j}$ deve ser identicamente nula sob $\Lambda$. Isto significa que, se $\phi_{j}$ denota a j-coordenada,

$$
\sum \xi_{j} d \phi_{j}(\xi)=0
$$

ou, se colocamos $H(\xi)=\sum \xi_{j} \phi_{j}(\xi)$, então

$$
d H(\xi)=\sum \phi_{j}(\xi) d \xi_{j}
$$

que é $\phi_{j}(\xi)=\frac{\partial H(\xi)}{\partial \xi_{j}}$, resultando (2.). Para provar que as coordenadas locais podem ser escolhidas para que (1.) seja cumprida precisamos do Lema 4.1 (para sua prova ver apêndice).

Prova de (1.): Escolhemos coordenadas locais $y$ tal que $\gamma_{0}=\left(0, \epsilon_{1}\right)$ com $\epsilon_{1}=$ $(1,0, \ldots, 0)$. O plano tangente para $\Lambda$ em $\gamma_{0}, V_{0}$, deve ser um plano lagrangeano. Se 
este é transverso ao plano $W=\left\{\left(y, \epsilon_{1}\right)\right\}=\left\{\left(y, d y_{1}\right)\right\}$, podemos tomar $y$ como as coordenadas dado que a transversalidade de $V_{0}$ e $W$ é equivalente a (1.). Se não, usamos o Lema 4.1 para escolher um plano lagrangeano $V$, o qual é transverso a ambos $V_{0}$ e $V_{1}=\{(0, \xi)\}$. A transversalidade de $V$ e $V_{1}$ significa que $V$ é uma secção passando através de $\gamma_{0}$ e daí $V=\left\{\left(y, d\left(y_{1}+Q(y)\right)\right)\right\}$ para alguma forma real quadrática $Q$. Se agora tomarmos $x_{1}=y_{1}+Q(y), \quad x_{j}=y_{j} \quad j=2, \ldots, n$ como novas coordenadas, segue que, nessas coordenadas, o plano tangente em $\gamma_{0}, \quad V_{0}$ e $V=\left\{\left(x, d x_{1}\right)\right\}$ são transversais.

Teorema 4.5 Sejam $\left(x_{0}, \theta_{0}\right) \in \mathbb{R}^{n} \times\left(\mathbb{R}^{N} \backslash\{0\}\right)$, $\Gamma$ uma vizinhança cônica suficientemente pequena de $\left(x_{0}, \theta_{0}\right)$, e $\phi$ uma função fase não degenerada numa vizinhança cônica aberta de $\left(x_{0}, \theta_{0}\right)$. Então, se $a \in S^{\mu}\left(\mathbb{R}^{n} \times \mathbb{R}^{N}\right), \quad \mu=m+$ $n / 4-N / 2$, é suportada em $\Gamma$, segue que

$$
u(x)=(2 \pi)^{-(n+2 N) / 4} \int_{\mathbb{R}^{N}} e^{i \phi(x, \theta)} a(x, \theta) d \theta \in I^{m}\left(\mathbb{R}^{n}, \Lambda\right)
$$

é uma distribuição lagrangeana $u(x) \in I^{m}\left(\mathbb{R}^{n}, \Lambda\right)$, com $\Lambda=\Lambda_{\phi}=\left\{\left(x, \phi_{x}^{\prime}(x, \theta)\right.\right.$ : $\left.(x, \theta) \in \Gamma_{\phi}\right\}$. Se as coordenadas são escolhidas tal que $\Lambda=\left\{\left(H^{\prime}(\xi), \xi\right\}\right.$, então

$$
\begin{gathered}
e^{i H(\xi)} \hat{u}(\xi)-(2 \pi)^{n / 4} a(x, \theta)\left|\operatorname{det} \phi^{\prime \prime}\right|^{-1 / 2} e^{\frac{\pi i}{4}} \operatorname{sgn} \phi^{\prime \prime} \\
\in S^{m-n / 4-1}
\end{gathered}
$$

para $|\xi|>1$ próximo de $\xi_{0}=\phi_{x}^{\prime}\left(x_{0}, \theta_{0}\right)$, onde $(x, \theta)$ é a solução do sistema $\phi_{\theta}^{\prime}(x, \theta), \quad \phi_{x}^{\prime}(x, \theta)=\xi e$

$$
\phi^{\prime \prime}=\left(\begin{array}{cc}
\phi_{x x}^{\prime \prime} & \phi_{x \theta}^{\prime \prime} \\
\phi_{\theta x}^{\prime \prime} & \phi_{\theta \theta}^{\prime \prime}
\end{array}\right)
$$

Reciprocamente, todo $u \in I^{m}\left(\mathbb{R}^{n}, \Lambda\right)$ com $W F(u)$ contido numa vizinhança pequena de $\left(x_{0}, \xi_{0}\right)$ pode escrever-se como

$$
u(x)=(2 \pi)^{-(n+2 N) / 4} \int_{\mathbb{R}^{N}} e^{i \phi(x, \theta)} a(x, \theta) d \theta
$$

$\in I^{m}\left(\mathbb{R}^{n}, \Lambda\right)$ módulo $C^{\infty}$. 
Este teorema é equivalente ao teorema de função de fase para operadores pseudodiferenciais, dado que a função fase $\varphi(x, y, \xi)$ do teorema da fase estacionária e a função fase $\langle x-y, \xi\rangle$ ambas parametrizam o lagrangeano trivial $\{(x, x, \xi,-\xi)\}$. Analogamente, se $u \in I_{\text {comp }}^{m}\left(\mathbb{R}^{n} \times \mathbb{R}^{n}, \Lambda\right)$, com $\Lambda$ o lagrangeano trivial, segue que $u(x, y)$ é o núcleo do operador pseudo-diferencial de ordem $m$.

Prova : Podemos assumir que $a(x, \theta)$ identicamente nula quando $x$ é exterior a um conjunto compacto e que as coordenadas têm sido escolhidas tais que $\Lambda=$ $\left\{\left(H^{\prime}(\xi), \xi\right\}\right.$. Usaremos fase estacionária para avaliar

$$
e^{i H(\xi)} \hat{u}(\xi)=(2 \pi)^{-(n+2 N) / 4} \iint e^{i[\phi(x, \theta)-<x, \xi>+H(\xi)]} a(x, \theta) d \theta d x .
$$

A primeira coisa que podemos ver é que o Herssiano (com respeito às variáveis de integração) da função de fase é não degenerado, isto é, $\operatorname{det} \phi^{\prime \prime} \neq 0$. Isto segue do fato que os mapas

$$
\Sigma_{\phi} \ni(x, \theta) \mapsto\left(x, \phi_{x}^{\prime}(x, \theta) \in \Lambda \quad \text { e } \quad \Lambda \ni(x, \xi) \mapsto \xi\right.
$$

são ambos difeomorfismos. Dado que $\phi_{\theta}^{\prime}=0$ sob $\Sigma_{\phi}$ isto significa que o mapa $\Gamma \ni(x, \theta) \mapsto\left(\phi_{x}^{\prime}, \phi_{\theta}^{\prime}\right)$ tem diferencias sobrejetivas sob $\Sigma_{\phi}$ e daí em $\Gamma$, se este conjunto é suficientemente pequeno. Isto vem do fato que $\operatorname{det} \phi^{\prime \prime} \neq 0$.

Dado que os pontos estacionários, dependentes do parâmetro $\xi$, são não degenerados, podemos assumir, possivelmente contraindo $\Gamma$, que, para todo $\xi$ próximo de $\xi_{0}$, existe um único ponto estacionário. Dado que podemos também assumir que $\phi_{x}^{\prime} \neq 0$ em $\Gamma$, segue que a diferença entre (4.7) e

$$
(2 \pi)^{-(n+2 N) / 4} \iint e^{i[\phi(x, \theta)-\langle x, \xi>+H(\xi)]} \beta\left(\frac{\theta}{|\xi|}\right) a(x, \theta) d \theta d x
$$

é rapidamente decrescente se $\beta \in C_{0}^{\infty}\left(\mathbb{R}^{n} \backslash\{0\}\right)$ é igual a 1 para $|\theta| \in\left[C^{-1}, C\right]$ com $C$ suficientemente grande, em particular, tal que $\beta\left(\frac{\theta}{|\xi|}\right)=1$ em um ponto estacionário. Se colocamos $\lambda=|\xi|$ e $w=\frac{\xi}{|\xi|}$, reescrevemos (4.8) como

$$
(2 \pi)^{-(n+2 N) / 4} \lambda^{N} \iint e^{i \lambda[\phi(x, \theta)-<x, w>+H(w)]} \beta(\theta) a(x, \lambda \theta) d \theta d x
$$

Note que a integração é sob um conjunto compacto fixo e que, em um ponto estacionário, temos $\phi_{\theta}^{\prime}=0, x=H^{\prime}(w)$ e daí, pelas relações homogêneas de Euler, $\phi(x, \theta)=<\phi_{\theta}^{\prime}(x, \theta), \eta>=0 \mathrm{e}<x, w>=<H^{\prime}(w), w>=H(w)$. Assim, 
chegamos à conclusão que a função de fase sempre é identicamente nula no ponto estacionário. Por isso a fórmula da fase estacionaria (2.28) diz que a diferença entre (4.8) e

$$
(2 \pi)^{n / 4} \lambda^{(N-n) / 2} a(x, \lambda \theta)\left|\operatorname{det} \phi^{\prime \prime}(x, \theta)\right|^{-1 / 2} e^{\frac{\pi i}{4}} \operatorname{sgn} \phi^{\prime \prime}
$$

está em $S^{m-n / 4-1}$, dado que $(m+n / 4-N / 2)+(N-n) / 2=m-n / 4$. Assim o $\operatorname{det} \phi^{\prime \prime}$ é homogêneo de grau $-(N-n)$ e também

$$
\lambda^{(N-n) / 2}\left|\operatorname{det} \phi^{\prime \prime}(x, \theta)\right|^{-1 / 2}=\left|\operatorname{det} \phi^{\prime \prime}(x, \lambda \theta)\right|^{-1 / 2} .
$$

Daí, (4.9) é um segundo termo em (4.6), o qual dá a primeira parte do teorema. Para provar o recíproco, usamos os teoremas 4.3 e 4.4 para ver que é suficiente considerar $u \in I^{m}\left(\mathbb{R}^{n}, \Lambda\right)$ tendo a propriedade que $v=\hat{u} e^{i H} \in S^{m-n / 4}$ é suportada numa vizinhança cônica pequena de $\xi_{0}$. Então colocamos $\Phi(x, \theta)=\frac{\partial \phi}{\partial x} \mathrm{e}$ $a_{0}(x, \theta)=(2 \pi)^{-n / 4} v \circ \Phi(x, \theta)\left|\operatorname{det} \phi^{\prime \prime}\right|^{1 / 2} e^{\frac{\pi i}{4}} \operatorname{sgn} \phi^{\prime \prime} \in S^{m+(n-2 N) / 4}$. Se definimos pelo análogo de (4.5) onde $a(x, \theta)$ é substituída por $a_{0}(x, \theta)$, segue que $u_{0}-u \in I^{m-1}$. Continuando, construímos $u_{j}$ de $a_{j}(x, \theta) \in S^{m+(n-2 N) / 4-j}$ tal que

$$
u-\left(u_{0}+\ldots+u_{j}\right) \in I^{m-j-1}\left(\mathbb{R}^{n}, \Lambda\right) .
$$

Se então escolhemos $a \sim \sum a_{j}$, segue que (4.6) mantém-se módulo $C^{\infty}$.

\subsection{Continuidade dos operadores integrais de Fou- rier}

Definição 4.13 Se $X$ e $Y$ são variedades $C^{\infty}$, então dizemos que um operador integral $F$ com kernell $K(x, y) \in I^{m}(X \times Y, \Lambda)$ é um operador integral de Fourier (FIO) de ordem $m$ se

$$
\Lambda \subset\left\{(x, y, \xi, \eta) \in T^{*}(X \times Y) \backslash\{0\}: \xi \neq 0, \eta \neq 0\right\} .
$$

Em termos da relação canônica temos

$$
C=\{(x, \xi, y, \eta): \quad(x, y, \xi,-\eta) \in \Lambda\} \subset\left(T^{*}(X) \backslash\{0\}\right) \times\left(T^{*}(Y) \backslash\{0\}\right),
$$

com notação $F \in I^{m}(X, Y, C)$. 
Dado $\Lambda$ lagrangiano com respeito à forma simplética $\sigma_{X}+\sigma_{Y}$, o sinal menos em (4.10) implica que $C$ é lagrangiano com respeito à forma simplética $\sigma_{X}-\sigma_{Y}=$ $\sum d \xi_{j} \wedge d x_{j}-\sum d \eta_{k} \wedge d y_{k}$ em $\left(T^{*}(X) \backslash\{0\}\right) \times\left(T^{*}(Y) \backslash\{0\}\right)$.

Definição 4.14 Uma relação canônica homogênea é chamada um gráfico canônico se para todo ponto $\gamma_{0}=\left(x_{0}, y_{0}, \xi_{0}, \eta_{0}\right) \in C$ existe um simplectomorfismo $\chi$ definido próximo de $\left(y_{0}, \eta_{0}\right)$ tal que, próximo de $\gamma_{0}, C$ é da forma

$$
\{(x, \xi, y, \eta): \quad(x, \xi)=\chi(y, \eta)\} .
$$

Note que isto faz com que a $\operatorname{dim} X=\operatorname{dim} Y$. Esta condição é equivalente à condição que a projeção $C \mapsto T^{*}(X) \backslash\{0\}$ ou $C \mapsto T^{*}(Y) \backslash\{0\}$ (e ambas) são localmente difeomorfismos. Se $C$ é localmente um gráfico canônico então as projeções são localmente difeomorfismos. Para ver o recíproco notemos que se $C \mapsto T^{*}(Y) \backslash\{0\}$, é um difeomorfismo local, então, próximo de $\gamma_{0}$, podemos usar $(y, \eta)$ como coordenadas para $C$ e daí a relação canônica deve localmente ser da forma (4.11). O fato que $\chi$ deve ser canônico é uma consequência do fato que $\sigma_{X}-\sigma_{Y}$ é identicamente nula sob $C$, o que faz $\sigma_{Y}=\chi^{*}\left(\sigma_{X}\right)$. Para uso futuro, expressamos esta condição em termos da função fase a qual localmente parametriza $C$. Se $\phi(x, y, \theta)$ é uma função fase não degenerada parametrizando $\Lambda$ então, por (4.10),

$$
C=\left\{\left(x, \phi_{x}^{\prime}(x, y, \theta), y,-\phi_{y}^{\prime}(x, y, \theta)\right):(x, y, \theta) \in \Sigma_{\phi}\right\} .
$$

Pretendemos que $C$ sendo localmente um gráfico seja equivalente à condição que

$$
\operatorname{det}\left(\begin{array}{cc}
\phi_{x y}^{\prime \prime} & \phi_{x \theta}^{\prime \prime} \\
\phi_{\theta y}^{\prime \prime} & \phi_{\theta \theta}^{\prime \prime}
\end{array}\right) \neq 0 \text { sob } \Sigma_{\phi} .
$$

Para ver isto, notemos que (4.12) é o jacobiano de $(y, \theta) \rightarrow\left(\phi_{x}^{\prime}, \phi_{\theta}^{\prime}\right)$. Também, se (4.12) mantém-se, então podemos resolver as equações

$$
\phi_{\theta}^{\prime}(x, y, \theta)=0, \quad \xi=\phi_{x}^{\prime}(x, y, \theta)
$$

com respeito a $(y, \theta)$ e daí usamos $(x, \xi)$ como coordenadas locais sob $\Sigma_{\phi}$. Assim, (4.12) é equivalente à condição de que a projeção $C \rightarrow T^{*}(X) \backslash\{0\}$ é um difeomorfismo local. 
A seguir vamos mostrar o teorema da composição que é o resultado principal que será usado na prova do teorema da continuidade em $L^{2}$ dos operadores integrais de Fourier.

\section{Teorema 4.6 (Composição de FIO)}

Sejam $F \in I_{\text {comp }}^{m}\left(X, Y, C_{1}\right)$ e $G \in I_{\text {comp }}^{\mu}\left(Y, Z, C_{2}\right)$. Então, se $C_{1}$ é localmente um gráfico de uma transformação canônica,

$$
F \circ G \in I^{m+\mu}(X, Z, C),
$$

onde

$$
\begin{aligned}
C=C_{1} \circ C_{2} & =\left\{(x, \xi, z, \zeta):(x, \xi, y, \eta) \in C_{1},\right. \\
& \left.e \quad(y, \eta, z, \zeta) \in C_{2} \quad \text { para algum } \quad(y, \eta) \in T^{*}(Y) \backslash\{0\}\right\} .
\end{aligned}
$$

Prova: Podemos assumir que $C_{1}$ é parametrizado por uma funçạa fase não degenerada $\phi(x, y, \theta)$ que é definida numa região canônica de $(X \times Y) \times\left(\mathbb{R}^{N_{1}} \backslash\{0\}\right)$ e que $C_{2}$ é parametrizado por uma função fase não degenerada $\varphi(y, z, \sigma)$ definida numa região canônica de $(Y \times Z) \times\left(\mathbb{R}^{N_{2}} \backslash\{0\}\right)$. Segue então que

$C_{1} \circ C_{2}=\left\{\left(x, \phi_{x}^{\prime}, z,-\varphi_{z}^{\prime}\right): \phi_{\theta}^{\prime}(x, y, \theta 5)=0, \varphi_{\sigma}^{\prime}(y, z, \sigma)=0, \quad-\phi_{y}^{\prime}(x, y, \theta)=\varphi_{y}^{\prime}(y, z, \sigma)\right\}$.

Equivalentemente, se colocamos

$$
\Theta=\left(\left(|\theta|^{2}+|\sigma|^{2}\right)^{1 / 2} y, \theta, \sigma\right)
$$

e definimos a função fase homogênea

$$
\Phi(x, z, \Theta)=\phi(x, y, \theta)+\varphi(y, z, \sigma)
$$

temos

$$
C_{1} \circ C_{2}=\left\{\left(x, \Phi_{x}^{\prime}, z,-\Phi_{z}^{\prime}\right):(x, z, \Theta) \in \Sigma_{\Phi}\right\} .
$$

Notemos que $\Phi$ é definida numa região canônica de $(X \times Z) \times\left(\mathbb{R}^{N} \backslash\{0\}\right)$, onde $N=N_{1}+N_{2}+n, \operatorname{com} n=\operatorname{dim} Y=\operatorname{dim} X$. Pretendemos mostrar que $\Phi$ seja não degenerada. Com isso provaremos que $C_{1} \circ C_{2}$ é uma relação canônica lagrangeana com respeito a $\sigma_{X}-\sigma_{Z}$. Para provar que $\Phi$ é não degenerada devemos mostrar que as diferenciais $d\left(\frac{\partial \Phi}{\partial \Theta_{j}}\right), \quad j=1, \ldots, N$, são linearmente independentes 
sob $\Sigma_{\Phi}$. Podemos expressar isto com o fato que a $N \times(N+n+\operatorname{dim} Z)$-matriz $\left(\frac{\partial^{2} \Phi}{\partial \Theta}\right) \partial(\Theta, x, z)$ tem posto total $N$. Isto significa que

$$
\left(\begin{array}{ccccc}
\phi_{y y}^{\prime \prime}+\varphi_{y y}^{\prime \prime} & \phi_{y \theta}^{\prime \prime} & \varphi_{y \sigma}^{\prime \prime} & \phi_{y x}^{\prime \prime} & \varphi_{y z}^{\prime \prime} \\
\phi_{\theta y}^{\prime \prime} & \phi_{\theta \theta}^{\prime \prime} & 0 & \phi_{\theta x}^{\prime \prime} & 0 \\
\varphi_{\sigma y}^{\prime \prime} & 0 & \varphi_{\sigma \sigma}^{\prime \prime} & 0 & \varphi_{\sigma z}^{\prime \prime}
\end{array}\right)
$$

tem posto total quando $\phi_{\theta}^{\prime}=0, \quad \varphi_{\sigma}^{\prime}=0$ e $\phi_{y}^{\prime}+\varphi_{y}^{\prime}=0$. Vimos que $C_{1}$ é localmente um gráfico, equivalente à condição que $\left(N_{1}+n\right) \times\left(N_{1}+n\right)$-submatriz

$$
\left(\begin{array}{ll}
\phi_{y \theta}^{\prime \prime} & \phi_{y x}^{\prime \prime} \\
\phi_{\theta \theta}^{\prime \prime} & \phi_{\theta x}^{\prime \prime}
\end{array}\right)
$$

é não singular quando $\phi_{\theta}^{\prime}=0 . \mathrm{O}$ fato que $\varphi$ é não degenerada faz

$$
\left(\begin{array}{lll}
\varphi_{\sigma y}^{\prime \prime} & \varphi_{\sigma \sigma}^{\prime \prime} & \varphi_{\sigma z}^{\prime \prime}
\end{array}\right)
$$

ter posto total $N_{2}$. Por combinação destes dois fatos e, notando a forma da matriz, vemos que (4.13) deve ter posto $N_{1}+n+N_{2}=N$. Usando partição da unidade, podemos assumir que $a_{1}(x, y, \theta) \in S^{m-N_{1} / 2+n / 2}$ e $a_{2}(y, z, \sigma) \in S^{\mu-N_{2} / 2+\left(n+n_{Z}\right) / 4}$ são suportadas em pequenos cones compactos em $\left(\mathbb{R}^{n} \times \mathbb{R}^{n}\right) \times\left(\mathbb{R}^{N_{1}} \backslash\{0\}\right.$ e $\left(\mathbb{R}^{n} \times\right.$ $\left.\mathbb{R}^{n_{z}}\right) \times\left(\mathbb{R}^{N_{2}} \backslash\{0\}\right.$, respectivamente. Aqui $n_{z}=\operatorname{dim} Z$. Devemos provar que se

$$
F(x, y)=\int_{\mathbb{R}^{N_{1}}} e^{i \phi(x, y, \theta)} a_{1}(x, y, \theta) d \theta
$$

e

$$
G(y, z)=\int_{\mathbb{R}^{N_{2}}} e^{i \varphi(y, z, \sigma)} a_{2}(y, z, \sigma) d \sigma
$$

então

$$
\begin{aligned}
F \circ G(x, z) & =\int F(x, y) G(y, z) d y \\
& =\iiint e^{i[\phi(x, y, \theta)+\varphi(y, z, \sigma)]} a_{1}(x, y, \theta) a_{2}(y, z, \sigma) d \theta d \sigma d y
\end{aligned}
$$

define um elemento de $I^{m}(X, Z, C)$. Se o conjunto frente de ondas de $y \mapsto F(x, y)$ e $y \mapsto \overline{G(y, z)}$ são caminhos diferentes, então o produto interno define um kernel $C_{0}^{\infty}$ e o resultado segue trivialmente. Se não, significa que, se $C$ é diferente do vazio e se assumimos, que os $a_{j}$ têm suporte cônico pequeno é claro do teorema 3.5 que o produto é bem definido. 
Para mostrar que $(F \circ G)(x, z)$ tem a forma desejada notemos que, se $\beta \in$ $C_{0}^{\infty}(\mathbb{R} \backslash\{0\})$ é igual a 1 para $s \in\left[C^{-1}, C\right]$ com $C$ suficientemente grande, e se colocamos

$$
a(x, z, y, \theta, \sigma)=\beta\left(\frac{|\theta|}{|\sigma|}\right) a_{1}(x, y, \theta) a_{2}(y, z, \sigma),
$$

então a diferença entre $(F \circ G)(x, z)$ e

$$
\iiint e^{i[\phi(x, y, \theta)+\varphi(y, z, \sigma)]} a(x, z, y, \theta, \sigma) d y d \theta d \sigma
$$

é $C^{\infty}$. Para provar isto notemos que, sob o suporte do integrando, se a soma $\phi_{y}^{\prime}+\varphi_{y}^{\prime}=0$ então $|\theta|$ e $|\sigma|$ devem ser comparáveis. Daí, se $C$ é suficientemente grande, $\left|\nabla_{y}(\phi+\varphi)\right|$ deve ser limitado por um múltiplo de $|\theta|+|\sigma|$ sob o suporte de $a-a_{1} a_{2}$.

Para finalizar, com uma mudança de variáveis escrevemos (4.14) como

$$
\int_{\mathbb{R}^{N}} e^{i[\Phi(x, y, \Theta)]} a(x, z, \Theta)\left(|\theta|^{2}+|\sigma|^{2}\right)^{-n / 2} d \Theta
$$

Note que, se $a_{1}$ e $a_{2}$ são símbolos de ordem $m-N_{1} / 2+n / 2$ e $\mu-N_{2} / 2+$ $\left(n+n_{z}\right) / 4$, respectivamente, e dado que o produto é outro símbolo de ordem $m+\mu-\left(N_{1}+N_{2}\right) / 2+3 n / 4+n_{z} / 4$ numa região canônica onde $|\theta|$ e $|\sigma|$ são comparáveis, concluímos que o símbolo em (4.15) é de ordem

$$
m+\mu-N / 2+\left(n+n_{z}\right) / 4 .
$$

Daí, concluimos que, pelo teorema 4.5, (4.15) define um elemento de $I^{m+\mu}(X, Z, C)$.

Vamos concluir o objetivo deste capítulo que é a prova do teorema de continuidade em $L^{2}$ dos operadores integrais de Fourier (FIO).

Teorema 4.7 Sejam $X$ e $Y$ variedades $n$-dimensionais $C^{\infty}$ e $F \in I_{\text {comp }}^{0}(X, Y, C)$, com $C$ localmente o gráfico da transformação canônica. Então

$$
F: L_{\text {comp }}^{2}(Y) \rightarrow L_{\text {comp }}^{2}(X)
$$

é contínuo. 
Prova : Se $F \in I_{\text {comp }}^{0}(X, Y, C)$, então $F^{*} \in I_{\text {comp }}^{0}\left(Y, X, C^{*}\right)$, onde $C^{*}=\{(y, \eta, x, \xi)$ : $(x, \xi, y, \eta) \in C\}=\{(y, \eta, x, \xi):(x, \xi)=\chi(y, \eta)\}$. Daí, $C \circ C^{*}$ deve ser a relação trivial $\{(y, \eta, y, \eta)\}$ e, pelo teorema 4.6 implica que $F^{*} F$ deve ser um $\psi D O$ de ordem zero. Pelo teorema 2.10 para um operador pseudo-diferencial $(\psi D O)$ de ordem zero temos

$$
\int|F u|^{2} d x=\int F^{*} F u \bar{u} d y \leq\left\|F^{*} F u\right\|_{2}\|u\|_{2} \leq C\|u\|_{2}^{2} .
$$

Corolário 4.1 Seja um operador integral de Fourier $F \in I_{\text {comp }}^{m}(X, Y, C)$ então

$$
F: H^{s} \mapsto H^{s-m},
$$

é um operador continuo, onde $H^{s}$ é o espaço de Sobolev com norma

$$
\|u\|_{s}^{2}=\int(1+|\xi|)^{2 s}|\hat{u}(\xi)|^{2} d \xi .
$$

Prova: Denotando

$$
h_{s}(\xi)=(1+\xi)^{s}
$$

podemos escrever

$$
\|u\|_{s}=\left\|h_{s}(D u)\right\|,
$$

e a afirmação do corolário que vamos mostrar fica

$$
\left\|h_{s-m}(D) F u\right\| \leq C\left\|h_{s}(D) u\right\| .
$$

Denotando $h_{s}(D) u$ como $v$, temos

$$
h_{s-m}(D) F u=h_{s-m}(D) F h_{-s}(D) v \in I_{\text {comp }}^{0}(X, Y, C),
$$

segue do teorema 4.7 que

$$
\left\|h_{s-m}(D) F h_{-s}(D) v\right\| \leq C\|v\| .
$$




\section{Capítulo 5}

\section{Parametriz de um operador hiperbólico de segunda ordem}

\subsection{Superfícies características, fluxo bicaracterístico}

\subsubsection{Superfícies características}

Superfícies características são as possíveis superfícies de propagação de descontinuidades das soluções das equações diferenciais parciais. Vamos supor 'que, localmente, a hiperfície $\Sigma$ seja descrita como a superfície de nível $\phi(x)=0$ de uma função $C^{\infty} \operatorname{com} \partial \phi \neq 0$.

Definição 5.1 O problema de Cauchy para uma EDP da forma:

$$
\sum_{j, k=0}^{n} a_{j k}(x) \partial_{x_{j}} \partial_{x_{k}}+\sum_{j=0}^{n} b_{j}(x) \partial_{x_{\jmath}}+c(x)=0
$$

consiste no seguinte: dados

1. Uma hiperfície $\Sigma$ de classe $C^{\infty}$ em $\mathbb{R}^{n} e$

2. funções $\left\{f_{\alpha}\right\}_{|\alpha| \leq m-1}$ definidas sobre $\Sigma$ e suficientemente diferenciáveis ao longo de $\Sigma$.

Determinar uma solução $u: \mathcal{U} \rightarrow \mathbb{R}$ de classe $C^{m}$ da equação (5.1), definida numa vizinhança $\mathcal{U}$ da hiperfície $\Sigma$ e tais que as derivadas parciais

$$
\left.\frac{\partial^{\alpha} u}{\partial x^{\alpha}}\right|_{\Sigma},
$$


quando calculadas ao longo da hiperfície $\Sigma$, coincidam com as funções $\left\{f_{\alpha}\right\}$ para todas as ordens $|\alpha| \leq m-1$.

Definição 5.2 Consideremos o operador linear de segunda ordem em $\mathbb{R}^{n+1}$

$$
P=\sum_{j, k=0}^{n} a_{j k}(x) \partial_{x_{j}} \partial_{x_{k}}+\sum_{j=0}^{n} b_{j}(x) \partial_{x_{j}}+c(x)
$$

Diremos que $\Sigma$ é uma hiperfície não-característica para $P$, se os valores das derivadas de maior ordem $\partial^{2} u$ forem determinados ao longo de $\Sigma$ univocamente pelos dados de Cauchy. Caso contrário diremos que a hiperfície $\Sigma$ é uma hiperfície característica para $P$.

Exemplo: $\Sigma=t: t=0$ é não-característica para o operador $L=\partial_{t}^{2}-a_{i j}(x) \partial_{i} \partial_{j}$.

\section{Definição $\mathbf{5 . 3}$}

$$
L=\sum_{j, k=0}^{n} a_{j k}(x) \partial_{x_{j}} \partial_{x_{k}}
$$

é chamada a parte principal do operador diferencial (5.2). Associamos ao operador $L$ a forma quadrática chamada de forma característica $Q(x,):. \mathbb{R}^{n+1} \times$ $\mathbb{R}^{n+1} \rightarrow \mathbb{R}$ definida por:

$$
Q(x, \xi)=\sum_{j, k=0}^{n} a_{j k}(x) \xi_{j} \xi_{k}
$$

Teorema 5.1 Uma superfície de nível $\xi(x)=0$ é característica se e só se

$$
Q(x, D \phi(x))=\sum_{i, j=1}^{n} a_{i j} \frac{\partial \xi}{\partial x_{i}} \frac{\partial \xi}{\partial x_{j}}=0 .
$$

Com isto, vemos que a existência de superfície característica está ligada à existência de zeros da forma característica.

Como a matriz $\left(a_{j k}\right)$ é simétrica e suas entradas são funções $C^{\infty}$, os seus autovalores são reais. Chamamos de assinatura da matriz $\left(a_{j k}\right)$ ao vetor $(k, l, n+1-k-l)$ definido pelo número $k$ de autovalores positivos, pelo número $l$ de autovalores nulos e pelo número restante $n+1-k-l$ de autovalores negativos da forma (5.4). Gostaríamos de examinar até que ponto a forma característica 
fornece-nos informações que independem do sistema de coordenadas. Uma mudança de coordenadas em $\mathbb{R}^{n+1}$ é um difeomorfismo

$$
\Phi: \mathbb{R}^{n+1} \mapsto \mathbb{R}^{n+1} .
$$

Para um tal difeomorfismo escrevemos $y=\Phi(x)$ e $\widetilde{u}(y)=u(x)$ para denotar a função $u$ nas novas coordenadas. Usando a regra da cadeia vemos que o operador (5.2) assume a nova forma

$$
\left(\Phi^{*} P\right) \widetilde{u}(y)=\sum_{i, j=0}^{n} \widetilde{a}^{i j}(y) \frac{\partial^{2} \widetilde{u}}{\partial y^{i} \partial y^{j}} \sum_{i=1}^{n+1} \widetilde{a}^{i}(y) \frac{\partial \widetilde{u}}{\partial y^{i}}+\widetilde{c}(y),
$$

onde os coeficientes da parte principal transformam-se de acordo com a fórmula

$$
\tilde{a}^{i j}=\sum_{\mu, \nu=0}^{n} a^{\mu, \nu} \frac{\partial \Phi^{i}}{\partial x^{\mu}} \frac{\partial \Phi^{j}}{\partial x^{\nu}} .
$$

Os termos de ordem inferior não importam para a discussão seguinte, já que não tomam parte na definição da forma característica. A observação de que a forma característica é um invariante do operador $P$ é dada pelo seguinte teorema.

Teorema 5.2 Para um operador linear de segunda ordem da forma (5.2) a forma característica (5.4) transforma-se covariantemente no espaço $T^{*} \mathbb{R}^{n+1}=\mathbb{R}^{n+1} \times$ $\left(\mathbb{R}^{n+1}\right)^{*}$. Isto é, para todo difeomorfismo $\Phi: \mathbb{R}^{n+1} \mapsto \mathbb{R}^{n+1}$, vale a seguinte relação

$$
Q(x . \xi)=Q\left(\Phi(x),\left[(D \Phi(x))^{T}\right]^{-1} \xi\right),
$$

para qualquer vetor $x \in\left(\mathbb{R}^{n+1}\right)$ e qualquer covetor $\xi \in\left(\mathbb{R}^{n+1}\right)^{*}$. Em particular, a assinatura da forma característica é um invariante do operador $P$ independente do sistema de coordenadas do espaço $\left(\mathbb{R}^{n+1}\right)$.

Prova: Observamos que, com $y=\Phi(x)$ e $\xi=(D \Phi(x))^{T} \eta$, temos

$$
\begin{aligned}
Q(y, \eta) & =\sum_{i, j=0}^{n} \widetilde{a}^{i j} \eta_{i} \eta_{j}=\sum_{i, j} \sum_{\mu, \nu}\left(a^{\mu, \nu} \frac{\partial \Phi^{j}}{\partial x^{\mu}} \frac{\partial \Phi^{j}}{\partial x^{\nu}}\right) \eta_{i} \eta_{j} \\
& \left.\left.=\sum_{\mu, \nu} a^{\mu, \nu} \sum_{i, j}\left(\frac{\partial \Phi^{j}}{\partial x^{\mu}} \eta_{i}\right)\left(\frac{\partial \Phi^{j}}{\partial x^{\nu}} \eta_{j}\right)=\sum_{\mu, \nu} a^{\mu, \nu}(D \Phi(x))^{T} \eta\right)_{\mu}(D \Phi(x))^{T} \eta\right)_{\nu} \\
& =\sum_{\mu, \nu} a^{\mu, \nu} \xi_{\mu} \xi_{\nu}=Q(x, \xi) .
\end{aligned}
$$




\section{Observações:}

1. Quando o operador $P$ tem coeficientes constantes então é possível encontrar uma transformação linear invertível $\Lambda: \mathbb{R}^{n+1} \mapsto \mathbb{R}^{n+1}$ para a qual a forma característica pode ser trazida para a forma diagonal e onde os autovalores assumem os valores 0 ou $\mp 1$. Isto pode ser obtido a partir de uma matriz ortogonal que diagonaliza a forma característica e por uma matriz de dilatação que dilata os eixos coordenados de forma a trazer os elementos da matriz diagonal à forma normal

$$
Q(\eta)=\sum_{i=0}^{n} k^{i} \eta_{i}^{2}
$$

$\operatorname{com} k^{i}=0$ ou $k^{i}=\mp 1$.

2. Quando os coeficientes do operador (5.2) não são mais constantes não é mais possível encontrar uma transformação linear como acima, de forma a trazer a forma característica à forma normal (5.6) simultaneamente para todos os pontos $x \in \mathbb{R}^{n+1}$. Note, no entanto, que se pré-fixamos um ponto $x \in \mathbb{R}^{n+1}$, então será sempre possivel encontrar uma tal transformação linear, neste caso dependendo do ponto $x$,

$$
\Lambda_{x}: \mathbb{R}^{n+1} \mapsto \mathbb{R}^{n+1}
$$

para a qual a forma característica assume, no ponto $x$, a forma normal (5.6) de assinatura $(k, l, n+1-k-l)$

$$
Q(x, \eta)=\eta_{0}^{2}+\eta_{1}^{2}+\ldots+\eta_{k}^{2}+0 . \eta_{k+1}^{2}+\ldots+0 . \eta_{k+l}^{2}-\eta_{k+l+1}^{2}-\ldots \eta_{n}^{2} .
$$

Definição 5.4 Considere um operador $P$ da forma (5.2) e um ponto $x \in\left(\mathbb{R}^{n+1}\right)$. Considere a transformação linear $\Lambda_{x}: \mathbb{R}^{n+1} \mapsto \mathbb{R}^{n+1}$ trazendo a forma característica $Q(x, \xi)$ no ponto $x$ para a forma normal (5.7). Diremos que o operador $P$ é:

1. Elíptico em $x$, se e somente se $k=n+1$ el $l=0$, isto é, a forma normal é dada por

$$
Q(x, \eta)=\eta_{0}^{2}+\eta_{1}^{2}+\ldots+\eta_{n}^{2}
$$


2. Parabólico em $x$, se e somente se $k=n$ e $l=1$, isto é, a forma normal é dada por

$$
Q(x, \eta)=\eta_{0}^{2}+\eta_{1}^{2}+\ldots+\eta_{n-1}^{2}+0 . \eta_{n}^{2} .
$$

3. Hiperbólico em $x$, se e somente se $k=n$ e $l=0$, isto é, a forma normal é dada por

$$
Q(x, \eta)=\eta_{0}^{2}+\eta_{1}^{2}+\ldots+\eta_{n-1}^{2}-\eta_{n}^{2} .
$$

Nestes três casos, em cada ponto, podemos examinar a questão do número de zeros da forma característica e determinar o número de superfícies características ligadas à EDP com forma característica (5.4). Vamos analisar isto para o caso hiperbólico: podemos ver que os zeros de (5.8) correspondem à uma família dupla de cones

$$
\eta_{n}= \pm\left(\eta_{0}^{2}+\eta_{1}^{2}+\ldots+\eta_{n-1}^{2}\right)^{1 / 2},
$$

denominados cones nulos. Eles dão origem no espaço físico $\mathbb{R}^{n+1}$ às duas famílias características descritas por cones $t= \pm|x|$ chamadas cones luminosos. Estes cones constituem o lugar geométrico por onde se propagam as singularidades, com a velocidade da luz.

\subsubsection{Método das bicaracterísticas:}

A teoria geral das EDP's não lineares da forma

$$
Q(x, p)=0, \quad p=D \phi
$$

conduz-nos ao estudo do sistema de equações ordinárias de forma Hamiltoniana chamadas de bicaracterísticas

$$
\begin{aligned}
& \frac{d x_{j}}{d t}=Q_{p_{j}} \\
& \frac{d p_{j}}{d t}=-Q_{x_{j}} \quad j=1 \ldots n .
\end{aligned}
$$

As curvas bicaracterísticas $(x(t), p(t)) \in \mathbb{R}^{2 n}$ determinam superfícies integrais em $\mathbb{R}^{n}$ que serão exatamente as superfícies características da equação original associada à equação do eikonal $Q(x, D \phi(x))=0$. 
Para um operador do tipo $L=\sum a_{j k} \partial_{j} \partial_{k}$, temos que

$$
Q(x, p)=\sum_{j, k=1}^{n} a_{j k}(x) p_{j} p_{k},
$$

e portanto

$$
\begin{aligned}
Q_{p_{l}} & =\sum_{j, k=1}^{n} a_{j k}(x) p_{j} \frac{\partial p_{k}}{\partial p_{l}}+\sum_{j, k=1}^{n} a_{j k}(x) p_{k} \frac{\partial p_{j}}{\partial p_{l}} \\
& =\sum_{j, k=1}^{n} a_{j k}(x) p_{j} \delta_{k l}+\sum_{j, k=1}^{n} a_{j k}(x) p_{k} \delta_{j l} \\
& =\sum_{j=1}^{n} a_{j l}(x) p_{j}+\sum_{k=1}^{n} a_{l k}(x) p_{k}
\end{aligned}
$$

se $a_{j k}=a_{k j}$ (a matriz $a_{j k}$ é simétrica). Daí,

$$
Q_{p_{l}}=2 \sum_{k=1}^{n} a_{l k}(x) p_{k} .
$$

Assim, o primeiro conjunto de equações em (5.10) reduz-se a

$$
\frac{d x_{j}}{d t}=2 \sum_{k=1}^{n} a_{j k}(x) p_{k} \quad j=1, \ldots, n .
$$

No caso especial onde as $a_{j k}$ são constantes, as bicaracterísticas de (5.1) são encontradas explicitamente. Se $a_{j k}=$ const, então $Q_{x_{j}}=0, \quad j=1, \ldots, n$. Daí a equação (5.10) diz que $\frac{d p_{j}}{d t}=0, \quad j=1 \ldots, n$, de tal modo os $p_{j}$ 's são constantes. Então (5.13) diz que os $\frac{d x_{j}}{d t}=$ const $, \quad j=1, \ldots, n$. Além disso,

$$
p^{(j)}=\sum_{k=1}^{n} a_{j k}(x) p_{k}
$$

então $\frac{d x_{j}}{d t}=p^{(j)}$ e, por isso, $x_{j}=p^{(j)} t+x_{0}^{(j)}$ onde os $x_{0}^{(j)}$ são constantes. Assim, quando os coeficientes das derivadas de segunda ordem são constantes, as bicaracterísticas são linhas retas. Mesmo se as $a_{j k}$ não são constantes, podemos ainda definir $p^{(j)}$ por (5.14), notando que agora $p^{(j)}$ será uma função de $x$. Ainda temos

$$
\frac{d x_{j}}{d t}=p^{(j)} \quad j=1, \ldots, n,
$$


só que agora estas equações não podem sempre ser integradas explicitamente. No entanto, se resolvemos (5.14) para $p^{(j)}$, temos que

$$
p_{j}=\sum_{k=1}^{n} b_{j k}(x) p^{(k)} \quad j=1, \ldots, n,
$$

onde $\left(b_{j k}\right)$ é a matriz inversa de $\left(a_{j k}\right)$. Substituindo (5.16) em (5.12) e simplificando encontramos

$$
Q(x, q)=\sum_{j, k=1}^{n} b_{j k}(x) p^{(j)} p^{(k)} .
$$

Para distinguir o vetor contravariante com componentes $p^{(j)}$ para o correspondente covariante com componentes $p_{j}$, usamos $q$ para denotar o contravariante, guardando $p$ para o covariante.

Usando (5.15) em (5.17), temos

$$
Q\left(x, \frac{d x_{j}}{d t}\right)=\sum_{j, k=1}^{n} b_{j k}(x) \frac{d x_{j}}{d t} \frac{d x_{k}}{d t} .
$$

Assim, a equação característica (5.9), que quando se escreve em termos de $p=D \phi$ determina as normais $p$ para a superfície característica $\phi(x)=0$, pode, por uso da matriz inversa da matriz de coeficientes, escrever-se como uma equação para os vetores tangentes $\frac{d x}{d t}$ às curvas bicaracterísticas $x(t)$; em outras palavras, 'uma direção característica $q$ é qualquer solução da equação

$$
\sum_{j, k=1}^{n} b_{j k}(x) p^{(j)} p^{(k)}=0,
$$

onde $\left(b_{j k}\right)=\left(a_{j k}\right)^{-1}$ e os $p^{(j)}$ são as componentes de $q$.

\subsection{Variedades Lorentzianas}

Nesta seção denotaremos por $L_{s}^{r}(V)$ o espaço de todos os tensores de tipo $(r, s)$ sobre $V$, isto é, o conjunto das aplicações multilineares. Quando $r=0$, $s=2$ denotamos o espaço $L_{0}^{2}$ como $B i(V)$, o espaço das formas bilineares simétricas sobre $V$. Vamos só considerar formas bilineares não-degeneradas, isto é, se $b(v, w)=0, \quad \forall w$, então $v=0$. O índice $\nu$ de uma forma bilinear simétrica é a dimensão do maior subespaço de $V$ onde a restrição da forma é negativa-definida. 
Definição 5.5 Um produto escalar Lorentziano num espaço vetorial $V$ é uma função bilinear simétrica não-degenerada de índice $\nu=1$. Em particular, existe uma base $\left\{e_{1}, e_{2}, \ldots, e_{n}\right\}$ em relação a qual a matriz de $b$ toma a forma

$$
b=\left(\begin{array}{cccc}
-1 & 0 & 0 & \ldots \\
0 & 1 & 0 & \ldots \\
\vdots & \vdots & \ddots & \vdots \\
0 & 0 & \ldots & 1
\end{array}\right) .
$$

Vamos agora globalizar estas noções, considerando os espaços tangentes $T_{p} M=$ $\mathbb{R}^{n}$ de uma variedade diferencial $M$ de dimensão $n$.

Definição 5.6 Um métrica Lorentziana $g$ (de classe $C^{\infty}$ ) numa variedade diferenciável $M^{n}$ é uma seç̧ão $C^{\infty}\left(M \mapsto L_{0}^{2}(M)\right)$ do fibrado de tensores simétricos do tipo $(0,2)$ tal que, para todo ponto $p \in M$, a forma bilinear $g_{p}: T_{p} M \times T_{p} M \mapsto$ $\mathbb{R}$ é um produto escalar Lorentziano.

Definição 5.7 Uma variedade Lorentziana é o par $(M, g)$ tal que o índice $\nu=1$ e $n \geq 2$.

Vamos usar $<,>$ como notação alternativa para $g$ e escrevermos $g(V, W)=<$ $V, W>\in T M$ para campos vetoriais. Se $x^{1}, \ldots, x^{n}$ é um sistema de coordenadas sobre $U \subset M$, as componentes do tensor métrico sob $U$ são $g_{i j}=<\partial_{i}, \partial_{j}>$ $(1 \leq i, \quad j \leq n)$. Assim para o campo vetorial $V=\sum V^{i} \partial_{i}$ e $W=\sum W^{j} \partial_{j}$,

$$
g(V, W)=<V, W>=\sum g_{i j} V^{i} W^{j} .
$$

Dado que $g$ é não degenerada, em cada ponto $p$ de $U$ a matriz $\left(g_{i j}(p)\right)$ é invertível, e esta matriz inversa é denotada por $\left(g^{i j}(p)\right)$. Como $g$ é simétrica, $g_{i j}=g_{j i}$ e daí $g^{i j}=g^{j i}$ para $1 \leq i, \quad j \leq n$. Finalmente sob $U$, pode-se escrever o tensor métrico como

$$
g=\sum g_{i j} d x_{i} \otimes d x_{j}
$$

Para cada $p \in \mathbb{R}^{n}$ existe um isomorfismo linear canônico de $\mathbb{R}^{n}$ em $T_{p}\left(\mathbb{R}^{n}\right)$ que, em termos de coordenadas naturais, envia $v$ a $v_{p}=\sum v^{i} \partial_{i}$. Assim, o produto ponto sob $\mathbb{R}^{n}$ dá origem a um tensor métrico sobre $\mathbb{R}^{n}$ com

$$
<v_{p}, w_{p}>=\sum v^{i} w^{i}
$$


Associamos ao produto escalar uma norma quadrada $\|v\|^{2}=\left\langle v_{p}, v_{p}\right\rangle$.

Um exemplo importante é o espaço $\mathbb{R}^{n+1}$ munido do produto interno

$$
<v, w>=-v^{0} w^{0}+\sum_{i=1}^{n} v^{i} w^{i} .
$$

Denotamos este espaço por $\left(\mathbb{R}^{n+1},<,>\right)$ e o chamamos de espaço de Minkowski. O significado geométrico do índice de uma variedade Rimanniana deriva-se da

Definição 5.8 Um vetor tangente $v$ de $M$ é

1. tipo espaço se $\|v\|^{2}>0$,

2. tipo luz ou nulo se $\|v\|^{2}=0$ e $v \neq 0$,

3. tipo tempo se $\|v\|^{2}<0$.

$O$ conjunto de todos os vetores nulos em $T_{p}(M)$ é chamado o cone de luz em $p \in M$.

\subsection{Parametriz de um operador hiperbólico de segunda ordem}

Estamos interessados em resolver o problema de Cauchy para a equação (5.2). Damos uma função $g(x, t)$ em $\Omega \times\left(T_{0}, T_{1}\right), \Omega$ aberto em $\mathbb{R}^{n}$ e funções de $x$, definidas em $\Omega, f_{j}(x)$. Procuramos uma função $u(x, t)$ em uma vizinhança $\Omega \times$ $\left(T_{0}, T_{1}\right)$ do hiperplano não característico $t=0$ satisfazendo

$$
\begin{gathered}
P\left(x, t, D_{x}, \partial_{t}\right) u=g \quad \text { em } \quad \Omega \times\left(T_{0}, T_{1}\right) \\
\left.\partial_{t}^{j}\right|_{t=0}=f_{j}(x) \text { em } \Omega .
\end{gathered}
$$

Gostaríamos de obter uma representação explícita para a solução do problema de Cauchy (5.20), ou uma aproximação desta solução dentro de uma certa regularidade. Fixemos um ponto $x_{0} \in \mathbb{R}^{n}$. Para cada inteiro $j>0$ queremos obter um operador $E=E_{j}$ tal que a composta $P o E$ é da forma

$$
P o E=I+R_{j},
$$


onde $R_{j}$ é um operador regularizante de ordem $-j$ em uma vizinhança do ponto $x_{0}$, isto é, denotando a bola de centro $x_{0}$ e raio $\delta>0$ por $B=B\left(x_{0}, \delta\right)$, então para cada $s$ o operador $R_{j}$ possui a propriedade regularizante

$$
R_{j}: H^{s}(B) \rightarrow H^{s+j}(B) \quad \forall s .
$$

Sintetizando o exposto acima, temos

Definição 5.9 Uma parametriz local (de regularidade $j$ ) de um operador $P$ numa vizinhança de $x_{0} \in \mathbb{R}^{n}$ é um operador $E_{j}: C_{0}^{\infty}(B) \rightarrow C_{0}^{\infty}(B)$ definido numa vizinhança $B \ni x_{0}$ tal que $P o E=I+R_{j}$ onde $R_{j}: H^{s}(B) \rightarrow H^{s+j}(B) \quad \forall s$. Quando $j=\infty$ dizemos que o resto $R_{j}$ é regularizante.

Quando $P$ é elíptico a existência da parametriz local é trivial, conforme vimos na proposição 2.2. Quando $P$ é hiperbólico a construção de uma parametriz local também é possível, porém um pouco mais complicada. Vamos considerar como hipótese desta seção só o caso que $P\left(D_{x}, \partial_{t}\right)$ seja estritamente hiperbólico, isto é,

Definição 5.10 O operador diferencial $P\left(D_{x}, \partial_{t}\right)$ de segunda ordem é estritamente hiperbólico se para cada $\xi \in \mathbb{R}^{n} \backslash\{0\}$ as raízes do polinômio $\tau \mapsto p(\xi, \tau)$

$$
p(\xi, \tau)=\tau^{2}+\left(\sum_{|\alpha|=1} a_{\alpha} \xi^{\alpha}\right) \tau+\left(\sum_{|\alpha|=2} a_{\alpha} \xi^{\alpha}\right)
$$

são puramente imaginárias e distintas, $i \lambda \mp(\xi)$.

A hipótese desta seção é que $P\left(D_{x}, \partial_{t}\right)$ seja estritamente hiperbólico. Dado que as raízes são distintas podemos ordená-las, digamos $\lambda_{-}<\lambda_{+}$. Cada uma delas é uma função analítica de $\xi$ em $\mathbb{R}^{n} \backslash\{0\}$, homogênea de grau um, isto é, $\lambda_{\mp}(s \xi)=s \lambda_{\mp}(\xi)$, $p\left(s \xi, i s \lambda_{\mp}(\xi)\right)=s^{2} p\left(\xi, i \lambda_{\mp}(\xi)\right)=0$ e $p\left(s \xi, i \lambda_{\mp}(s \xi)\right)=0$. Além disto, para alguma constante $C_{0}>0, C_{0}|\xi| \leq\left|\lambda_{+}(\xi)-\lambda_{-}(\xi)\right| \quad \forall \xi \in \mathbb{R}^{n}$

Pode-se mostrar agora o seguinte teorema

\section{Teorema 5.3 (Parametriz local)}

Se $P$ é um operador diferencial hiperbólico com símbolo $p(x, \xi) \in S^{m}\left(\mathbb{R}^{n} \times \mathbb{R}^{n}\right)$, então existe uma parametriz local $E$ com resto em $S^{-\infty}$. E é da forma

$$
E=\sum F I O,^{1}
$$

com função fase $\phi$ e amplitudes $a_{j k l}$ determinadas pelo operador $P$.

\footnotetext{
${ }^{1}$ no capítulo anterior denotamos o operador integral de Fourier por FIO
} 


\section{Prova: Caso 1-coeficientes constantes}

Vamos considerar inicialmente o caso quando os coeficientes do operador homogêneo (5.20) são constantes (podemos denotar por $P\left(D_{x}, \partial_{t}\right)$ o operador (5.20)), em (5.21) os $f_{j}$ são funções $C^{\infty}$ com suporte compacto em $\Omega$. Depois analisaremos o caso de coeficientes variáveis.

$\mathrm{O}$ procedimento vai ser analisado para o operador de onda. Consideremos $P\left(\partial_{t}, D x\right)=\partial_{t}^{2}-a^{i j} \partial_{x i} \partial_{x j}$, onde $A=\left(a^{i j}\right) \geq 0$ é uma matriz simétrica por definição e de coeficientes constantes. Assim,

$$
\begin{gathered}
u_{t t}-a^{i j} \partial_{x i} u \partial_{x j} u=0, \\
\left.\partial_{t}^{j} u\right|_{t=0}=f_{j}(x) .
\end{gathered}
$$

Neste caso, tomando a transformada de Fourier, temos que

$$
\begin{gathered}
\hat{u}_{t t}+\left(a^{k l} \xi_{k} \xi_{l}\right) \hat{u}=0 \\
\left(\frac{d^{j}}{d t^{j}}\right) \hat{u}(\xi, 0)=\hat{f}_{j}(\xi) .
\end{gathered}
$$

A equação (5.24) é uma equação diferencial ordinária, linear e com coeficientes constantes que passam a depender do parâmetro $\xi$. De fato, a solução de (5.24)-(5.25) existe e é única. Ela também depende só de $\xi$, isto é, ela pode ser estendida para valores complexos de $\xi$ em $\mathbb{C}^{n}$ como uma função inteira de tipo exponencial (de fato, dependendo só de $t, T_{0}<t<T_{1}$ ). Isto significa que, se $\hat{u}(\xi, t)$ é transformada de Fourier com respeito a $x$ de uma distribuição, ela pode ter suporte compacto. A dificuldade, se existe, é achar as raízes do polinômio $p(\xi, \tau)$ na variável $\tau$. Vejamos o comportamento da solução $\hat{u}(\xi, t)$ de (5.24)(5.25) quando $\xi \in \mathbb{R}^{n}$. Podemos escrever

$$
\hat{u}(\xi, t)=\sum_{j=0}^{1} \widetilde{E_{j}}(\xi, t) \widehat{f}_{j}(\xi),
$$

onde, para cada $j=0,1, \widetilde{E_{j}}(\xi, t)$ é solução única do seguinte problema

$$
\left(\frac{d^{2}}{d t^{2}}\right) \widetilde{E_{j}}+\left(a^{k l} \xi_{k} \xi_{l}\right) \widetilde{E_{j}}=0 \quad \forall t \in \mathbb{R}
$$




$$
\left.d_{t}^{k} \widetilde{E_{j}}\right|_{t=0}=\left\{\begin{array}{lll}
1 & \text { se } & j=k \\
0 & \text { se } & j \neq k
\end{array}\right.
$$

Se precisamos que $\hat{u}(\xi, t)$ seja temperada para $\xi \in \mathbb{R}^{n}$ qualquer que seja $f_{j}$, é necessário e suficiente que $\widetilde{E_{j}}(\xi, t)$ seja temperada para $\xi \in \mathbb{R}^{n}$ e para todo $j=0,1$.

Denotamos por $i \lambda_{\mp}(\xi)$ as raízes do polinômio em $\tau, p(\xi, \tau)=\tau^{2}+q(\xi)=$ $\tau^{2}+a^{k l} \xi_{k} \xi_{l}$ para $\xi$ fixo arbitrário. Observemos que

$$
h_{\mp}(\xi, t)=e^{i t \lambda_{\mp}(\xi)}
$$

são soluções do problema (5.24)-(5.25) com dados de Cauchy $\left[i \lambda_{\mp}(\xi)\right]^{j}$ e por $(5.26)$ temos que

$$
h_{\mp}(\xi, t)=\sum_{+,-} \widetilde{E_{j}}(\xi, t) \tau_{\mp}(\xi)^{j} .
$$

Quando as raízes $\tau_{\mp}$ são distintas de (5.29)-(5.30) temos

$$
\left[\begin{array}{cc}
1 & \tau_{-}(\xi) \\
1 & \tau_{+}(\xi)
\end{array}\right]\left[\begin{array}{c}
\widetilde{E_{0}}(\xi, t) \\
\widetilde{E_{1}}(\xi, t)
\end{array}\right]=\left[\begin{array}{c}
e^{t \tau_{-}(\xi)} \\
e^{t \tau_{+}(\xi)}
\end{array}\right]
$$

Portanto, podemos achar a solução de (5.30) como segue. Seja $V\left(\tau_{-}, \tau_{+}\right)$e $V_{j}\left(\tau_{-}, \tau_{+}, t\right)$ os determinantes de Vandermonde em $\tau_{\mp}$, onde $V_{j}\left(\tau_{-}, \tau_{+}, t\right)$ tem as raízes $\tau_{-}^{j}, \tau_{+}^{j}, \quad j=0,1$ substituídas por $e^{t \tau_{-}}, e^{t \tau_{+}}$, isto é,

$$
\begin{gathered}
V_{0}\left(\tau_{-}, \tau_{+}, t\right)=\operatorname{det}\left[\begin{array}{cc}
e^{t \tau_{-}(\xi)} & \tau_{-}(\xi) \\
e^{t \tau_{+}(\xi)} & \tau_{+}(\xi)
\end{array}\right] \\
V_{1}\left(\tau_{-}, \tau_{+}, t\right)=\operatorname{det}\left[\begin{array}{ll}
1 & e^{t \tau_{-}(\xi)} \\
1 & e^{t \tau_{+}(\xi)}
\end{array}\right] .
\end{gathered}
$$

Então

$$
\widetilde{E_{j}}(\xi, t)=V_{j}\left(\tau_{-}, \tau_{+}, t\right) / V\left(\tau_{-}, \tau_{+}\right) .
$$

No caso da equação da onda em $\mathbb{R}^{n+1}$,

$$
\widetilde{E_{0}}(\xi, t)=\frac{1}{2}\left(e^{i t|\xi|}+e^{-i t|\xi|}\right) \quad \text { e } \widetilde{E_{1}}(\xi, t)=\frac{1}{2 i|\xi|}\left(e^{i t|\xi|}-e^{-i t|\xi|}\right) .
$$


Definamos os operadores

$$
\begin{aligned}
E_{j}(t) u(x) & =(2 \pi)^{-n} \int e^{i x \cdot \xi} \widetilde{E_{j}}(\xi, t) \hat{u}(\xi) d \xi, \quad u \in C_{c}^{\infty}\left(\mathbb{R}^{n}\right) \\
& =\sum_{+,-}(2 \pi)^{-n} \int_{|\xi|>M} e^{i\left(x \cdot \xi+t \lambda_{\mp}(\xi)\right)} a_{j \mp}(\xi, t) \hat{u}(\xi) d \xi \\
& +(2 \pi)^{-n} \int_{|\xi| \leq M} e^{i x \cdot \xi \widetilde{E_{j}}(\xi, t) \hat{u}(\xi) d \xi .}
\end{aligned}
$$

Módulo um operador regularizante, $E_{j}(t)$ é igual à soma de 2 FIO da forma

$$
F(t) u(x)=(2 \pi)^{-n} \int e^{i \phi(x, t, \xi)} a(\xi, t) \hat{u}(\xi) d \xi,
$$

onde $a(\xi, t)$ é uma função $C^{\infty}$ de $(\xi, t)$ em $\mathbb{R}^{n} \times \mathbb{R}$ e

$$
\phi(x, t, \xi)=x \cdot \xi+t \lambda(\xi),
$$

onde $\lambda(\xi)$ é analítica e homogênea de grau um em $\mathbb{R}^{n} \backslash\{0\}$ e $i \lambda(\xi)$ é uma raiz do polinômio em $\xi, P_{m}(\xi, \tau)$, o que significa que a função $\phi$ é a solução da equação característica

$$
P_{m}\left(\partial_{x} \phi, i \partial_{t} \phi\right)=0
$$

onde $\partial_{x} \phi=\operatorname{grad}_{x} \phi$. Além disso, a função $\phi$ satisfaz a condição de Cauchy .

$$
\left.\phi\right|_{t=0}=x . \xi .
$$

A equação (5.35) é de primeira ordem mas não linear; então isto se divide em duas equações da forma

$$
\partial_{t} \phi=\lambda_{k}\left(\partial_{x} \phi\right), \quad k=1,2 .
$$

Cada problema (5.36)-(5.3i) tem uma única solução suave, que é real, e (5.34) é a solução de um dos problemas.

\section{Caso 2-coeficientes variáveis}

Vamos estender para o operador de onda com coeficientes variáveis o método usado para coeficientes constantes. Consideremos operadores integrais de Fourier $E_{j} \in L^{-2}$ da forma

$$
\left[E_{j} f\right](x)=\int_{\mathbb{R}^{n}} e^{i \phi(x, \xi)} a_{j k}(x, \xi) \hat{f}(\xi) d \xi,
$$


onde $a_{j k} \in S^{-2}$ é um símbolo e $\phi$ é uma função de fase não-degenerada em $\mathbb{R}^{n} \times\left(\mathbb{R}^{n} \backslash\{0\}\right)$, isto é,

$$
\frac{\partial^{2} \phi}{\partial_{x} \partial_{\xi}} \neq 0
$$

A parametriz pode ser construída explicitamente a partir de um conjunto de equações diferenciais sobre o símbolo do operador. Seja $p \sim \sum_{j} p_{j}$ a expansão assintótica do símbolo $p(x, \xi)$. A solução $a_{j k}(x, \xi)=\sum_{l} a_{j k l}$ é construída recursivamente calculando a ação de $P$ sob o operador (5.38). Para uma função $a \in C^{\infty}$ qualquer devemos investigar a ação de $P$ em funções oscilatórias,

$$
P\left(e^{i \phi} a\right)=e^{i \phi} P\left(x, \partial_{x}+i \partial \phi\right) a
$$

O trabalho é determinar os símbolos $\phi_{k}$ e $a_{j k}$ tais que

$$
P\left(e^{i \phi} a_{j k}\right) \in S^{-\infty}
$$

De (5.32), temos que $E_{j}$ pode ser representada por um FIO. Mais especificamente, em coordenadas locais para $t$ pequeno, temos

$$
\left[E_{j} f\right](x)=\sum_{k=1}^{2}(2 \pi)^{-n} \int e^{i \phi_{k}(x, \xi)} a_{j k}(x, \xi) \hat{f}(\xi) d \xi+R_{j} f(x),
$$

onde $R_{j}$ é um operador regularizante.

Seja

$$
P=a^{\alpha \beta} \partial_{\alpha} \partial_{\beta}+b^{\alpha} \partial_{\alpha}+c(x)
$$

$\operatorname{com} a^{00}=-1, a^{i j}=a^{i j}(x)$.

$$
P(x, \partial x) E_{j} u(x) \sim \sum_{k=1}^{2}(2 \pi)^{-n} \int e^{i \phi_{k}(x, \xi)} P\left(x, \partial_{x}+i \partial \phi\right) a_{j k}(x, \xi) \hat{u}(\xi) d \xi
$$

módulo um operador regularizante.

Precisamos que, para cada $k=1,2$,

$$
P\left(x, \partial_{x}+i \partial \phi\right) a_{j k}(x, \xi)=0,
$$

isto é,

$a^{\alpha \beta} \partial_{\alpha} \partial_{\beta} a_{j k}+a^{\alpha \beta} i \phi_{\beta} \partial_{\alpha} a_{j k}+a^{\alpha \beta} i \phi_{\alpha} \partial_{\beta} a_{j k}-a^{\alpha \beta} \phi_{\alpha} \phi_{\beta} a_{j k}+b^{\alpha} \partial_{\alpha} a_{j k}+i b^{\alpha} \phi_{\alpha} a_{j k}+c(x) a_{j k}=0$. 
Os termos homogêneos com respeito a $\xi$ em (5.41) são todos identicamente nulos. O termo com máximo grau de homogeneidade é $P_{m}(x, i \partial \phi) a_{j k 0}$ portanto, precisamos resolver a equação da eikonal

$$
P_{m}(x, i \partial \phi)=0
$$

isto é,

$$
\partial_{t} \phi^{ \pm}= \pm\left(a^{i j} \phi_{x_{i}} \phi_{x_{j}}\right)^{1 / 2}
$$

com condição inicial

$$
\phi(0, x)=x . \xi .
$$

Como a equação da eikonal (5.42) é uma EDP não linear de primeira ordem, podemos solucioná-la pelo método das bicaracterísticas e uma das soluções é a função fase $\phi_{k}$.

Vamos determinar os símbolos $a_{j k l}$, resolvendo as equações de transporte (5.39), não podemos esperar que estas equações sejam independentes de $x$. Primeiro, escolhemos $a_{j k 0}$ tal que, para $t=x_{0}$ pequeno e $\xi$ grande, temos,

$a^{\alpha \beta} \partial_{\alpha} \partial_{\beta} a_{j k 0}+2 i a^{\alpha \beta} \phi_{\beta} \partial_{\alpha} a_{j k 0}-a^{\alpha \beta} \phi_{\alpha} \phi_{\beta} a_{j k 0}+b^{\alpha} \partial_{\alpha} a_{j k 0}+i b^{\alpha} \phi_{\alpha} a_{j k 0}+c(x) a_{j k 0}=0$

$\mathrm{e}$

$$
a_{j k 0}\left(0, x^{\prime}, \xi\right)=I(x, \xi) .
$$

Portanto,

$$
2 i a^{\alpha \beta} \phi_{\beta} \partial_{\alpha} a_{j k 0}+i b^{\alpha} \phi_{\alpha} a_{j k 0}=-a^{\alpha \beta} \partial_{\alpha} \partial_{\beta} a_{j k 0}-b^{\alpha} \partial_{\alpha} a_{j k 0}-c(x) a_{j k 0},
$$

onde o operador de transporte é dado por

$$
\mathcal{T}=2 i a^{\alpha \beta} \phi_{\beta} \partial_{\alpha}+i b^{\alpha} \phi_{\alpha}
$$

Os $a_{j k l}$ são determinados por indução. Vamos supor que $l=0,1, \ldots, N$, pois o resto é um operador regularizante. Então,

$$
(P(x, D)+\mathcal{T})\left(a_{j k 0}+a_{j k 1}+\ldots+a_{j k N}\right)=0 .
$$

Isto é,

$$
P(x, D) a_{j k 0}+\mathcal{T} a_{j k 0}=0
$$




$$
\begin{gathered}
P(x, D) a_{j k 1}+\mathcal{T} a_{j k 1}=0 \\
\vdots \\
P(x, D) a_{j k(N-1)}+\mathcal{T} a_{j k(N-1)}=0 \\
P(x, D) a_{j k N}+\mathcal{T} a_{j k N}=0 .
\end{gathered}
$$

Usando o método de aproximações sucessivas, temos que

$$
\mathcal{T} a_{j k l}=-a^{\alpha \beta} \partial_{\alpha} \partial_{\beta} a_{j k(l-1)}-b^{\alpha} \partial_{\alpha} a_{j k(l-1)}-c(x) a_{j k(l-1)}=-P a_{j k(l-1)} .
$$

As equações (5.44) não são por si mesmas suficientes para determinar os $a_{j k l}$. Vamos juntar condições apropriadas no tempo $t=x_{0}=0$. Usando (5.40), obtemos

$$
\partial_{x_{0}}{ }^{r} E_{j} f(x) \sim \sum_{k=1}^{2}(2 \pi)^{-n} \int e^{i \phi_{k}(x, \xi)}\left(\partial_{x_{0}}+i \partial_{x_{0}} \phi_{k}\right)^{r} a_{j k}(x, \xi) \hat{f}(\xi) d \xi .
$$

Precisamos, para $r=0,1$, que

$$
\left.\sum_{k=1}^{2}\left(\partial_{x_{0}}+i \partial_{x_{0}} \phi_{k}\right)^{r} a_{j k}(x, \xi)\right|_{x_{0}=0}=\left\{\begin{array}{lll}
1 & \text { se } & j=r \\
0 & \text { se } & j \neq r .
\end{array}\right.
$$

O termo com maior grau de homogeneidade é

$$
\left.\sum_{k=1}^{2}\left(i \partial \phi_{k}\right)^{r} a_{j k 0}\right|_{x_{0}=0}
$$

Observemos que quando $t=x_{0}=0, \partial_{t} \phi_{k}=\lambda_{k}$ por (5.42). Portanto,

$$
\left.\sum_{k=1}^{2}\left(i \lambda_{k}\right)^{r} a_{j k 0}\right|_{t=0}=\left\{\begin{array}{lll}
1 & \text { se } & j=r \\
0 & \text { se } & j \neq r .
\end{array}\right.
$$

Novamente introduzimos o determinante de Vandermonde $V\left(\lambda_{1}, \lambda_{2}\right)$ e o denotamos por $V_{j k}\left(\hat{\lambda}_{k}\right)$, com $j=0,1$ e $k=1,2$, o menor de $V\left(\lambda_{1}, \lambda_{2}\right)$, isto é, $V_{j 1}\left(\hat{\lambda}_{1}, \lambda_{2}\right)$ e $V_{j 2}\left(\lambda_{1}, \hat{\lambda}_{2}\right)$. Então, da equação $(5.47)$, temos que

$$
a_{j k 0}=V_{j k}\left(\hat{\lambda}_{k}\right) / V\left(\lambda_{1}, \lambda_{2}\right) \text { quando } t=0 .
$$




\section{Capítulo 6}

\section{O teorema de propagação de singularidades}

Com ajuda do conjunto frente de ondas mostraremos, neste capítulo, o teorema de propagação de singularidades das soluções de $P u=f$, que é o objetivo desta dissertação. Este resultado é devido a Hörmander (9).

Teorema 6.1 Seja p o símbolo principal do operador diferencial parcial $P$ tal que $p$ é real e u é uma solução de $P u=f \in C^{\infty}$. Suponhamos que $\left(x_{0}, \xi_{0}\right) \in W$. Fu. Vamos assumir que $\operatorname{grad}_{\xi} p\left(x_{0}, \xi_{0}\right) \neq 0$, tal que a bicaracterística nula através de $\left(x_{0}, \xi_{0}\right)$ é bem definida próxima de $\left(x_{0}, \xi_{0}\right)$. Denotamos por $\Gamma$ a extensão maximal da bicaracterística nula. Então toda a curva $\Gamma \in W F u$.

Vamos provar que, se um ponto $(\bar{x}, \bar{\xi}) \in \Gamma$ não pertence ao conjunto frente de ondas de u, então toda a curva $\Gamma$ não está no conjunto frente de ondas de $u$. Podemos assumir que $u$ tem suporte compacto. Precisamos provar o resultado só localmente sobre $\Gamma$, pois o argumento pode ser continuado ao longo de $\Gamma$; isto é uma das vantagens de se trabalhar em espaços cotangentes.

Prova: Suponhamos $(\bar{x}, \bar{\xi}) \notin \Gamma$ e $\left(\frac{\partial}{\partial \xi_{n}}\right) p(\bar{x}, \bar{\xi}) \neq 0$. Então, pela observação do lema 3.2, existe um operador pseudo-diferencial $A$ de grau zero com $A u \in C^{\infty}$ e o símbolo $a(x, \xi)=1$ numa vizinhança de $(\bar{x}, \bar{\xi})$. Nosso objetivo é construir um operador pseudo-diferencial $B$ de grau zero tal que $B u \in C^{\infty}$ e $b_{0}(x, \xi) \neq 0$ ao longo de $\Gamma$, sendo $b_{0}$ o símbolo principal de $B$. Pelo Lema 3.2 isto prova o teorema. 
$B$ é construída com $b_{0} \neq 0$ sobre $\Gamma$ tal que $[P, B]$ é $C^{\infty}$, isto é, tem símbolo identicamente nulo, e o suporte do símbolo $b$ de $B$ fica numa vizinhança cônica de $\Gamma$, e tal que $B u \in C^{\infty}$ próximo do plano $x^{n}=\bar{x}^{n}$. Para realizar isto desejamos determinar o símbolo $b=b_{0}+b_{-1}+\ldots$, onde $b_{-j}$ é de ordem $-j$. O símbolo principal de $[P, B]$ é

$$
\frac{1}{i} H_{p} b_{0}=\frac{1}{i} \sum\left(\frac{\partial p}{\partial \xi_{j}} \frac{\partial b_{0}}{\partial x^{j}}-\frac{\partial p}{\partial x^{j}} \frac{\partial b_{0}}{\partial \xi_{j}}\right) .
$$

Vamos escolher $b_{0}$ para ser solução de $\frac{1}{i} H_{p} b_{0}=0$, tal que $b_{0}$ é constante sobre as bicaracterísticas de $p$. Podemos achar tal $b_{0} \operatorname{com} b_{0}(\bar{x}, \bar{\xi})=1$ e tal que $b_{0}$ tenha suporte numa vizinhança cônica pequena de $\Gamma$ (usando a homogeneidade de $p$ ). Podemos prescrever $b_{0}$ sobre uma hiperfície transversal para as bicaracterísticas através de $(\bar{x}, \bar{\xi})$, com $b_{0}$ identicamente nulo fora de uma vizinhança pequena de $(\bar{x}, \bar{\xi})$ e homogênea de grau um em $\xi$. Podemos assegurar que, para $x^{n}$ próximo de $\bar{x}^{n}, a=1$ sobre o suporte de $b_{0}$. Agora todos os demais termos $b_{-j}$, podem ser determinados com suporte em alguma vizinhança cônica pequena de $\Gamma$ tal que o símbolo de $[P, B]$ é zero. Por exemplo, para achar $b_{-1}$ olhamos todos os termos de ordem $m-2$; se $p(x, \xi)=p+p_{m-1}+\ldots$ precisamos

$$
\frac{1}{i} H_{p} b_{1}+\frac{1}{i} H_{p_{m-1}} b_{0}+\sum_{|\alpha|=2} \frac{1}{\alpha !}\left(\partial_{\xi}^{\alpha} p_{m} D_{x}^{\alpha} b_{0}-\partial_{\xi}^{\alpha} b_{0} D_{x}^{\alpha} p_{m}\right)=0 .
$$

Isto produz uma equação diferencial ordinária para $b_{-1}$ ao longo de cada curva bicaracterística de $p$, a qual pode ser resolvida. Analogamente para cada $b_{-j}$.

Assim, podemos inferir que $P B u=B P u+[P, B] u \in C^{\infty}$, dado que ambos os termos $B P u$ e $[P, B] u$ pertencem a $C^{\infty}$. Além disso, para $x^{n}$ próximo de $\bar{x}^{n}$ o símbolo de $A$ é identicamente um sobre o suporte de $b$ tal que o símbolo de $B(I-A)$ é identicamente nulo. Por isso, para $x^{n}$ próximo de $\bar{x}^{n}$,

$$
B u=B(I-A) u+B A u \in C^{\infty} .
$$

Em uma vizinhança de $(\bar{x}, \bar{\xi})$ o símbolo de $p$ pode ser fatorado como

$$
p=\left(\xi_{n}-\lambda\left(x, \xi_{1}, \ldots, \xi_{n-1}\right)\right) q
$$

com $\lambda$ real, homogênea em $\xi^{\prime}=\left(\xi_{1}, \ldots, \xi_{n-1}\right)$ de grau um e $q(\bar{x}, \bar{\xi}) \neq 0$. Aplicando a proposição 2.3 podemos achar um operador hiperbólico

$$
L=D_{n}-\lambda\left(x, D_{1}, \ldots, D_{n-1}\right)-\rho_{0}\left(x, D_{1}, \ldots, D_{n-1}\right),
$$


e um operador elíptico $Q(x, D)$, tal que o símbolo de $P-L Q$ seja identicamente nulo sobre o suporte dos termos no símbolo de $B$. Por isso $L Q B u \in C^{\infty}$. Dado que $B u \in C^{\infty}$ para $x^{n}$ próximo de $\bar{x}^{n}$ também $Q B u \in C^{\infty}$. Como $L$ é um operador hiperbólico sabemos que o problema de Cauchy é bem posto e existe uma única solução $u \in C^{\infty}$ se os dados iniciais são $C^{\infty}$. Assim, segue que $Q B u \in C^{\infty}$ e, como $Q$ é elíptico da mesma forma, concluímos que $B u \in C^{\infty}$.

Exemplo: Vamos provar que a equação da onda $\frac{\partial^{2} u}{\partial x_{0}^{2}}-\Delta u=0 \mathrm{em} \mathbb{R}^{n+1}$ não pode ter soluções com singularidades isoladas. No exemplo temos $m=2$, e assim $p_{2}(x, \xi)=-\xi_{0}^{2}+|\xi|^{2}$. O sistema hamiltoniano

$$
\begin{aligned}
& \dot{\xi}=-\frac{\partial p_{2}}{\partial x}=0 \\
& \dot{x}=\frac{\partial p_{2}}{\partial \xi}=\left\{\begin{array}{l}
-2 \xi_{0} \\
2 \xi
\end{array}\right.
\end{aligned}
$$

tem a solução $\xi=$ constante, $\quad x_{o}=-2 \xi_{0} t, \quad x=2 \xi t$. Seja $0 \in \operatorname{suppsing}(u)$. Então existe um ponto $\left(0,0, \xi_{0}, \xi\right) \in W F(u)$, tal que pelo Lema 3.2 temos que $|\xi|^{2}=\xi_{0}^{2}$. Pelo teorema $6.1\left(-2 \xi_{0} t, 2 \xi t, \xi_{0}, \xi\right) \in W F(u)$ para qualquer $t$, em particular, $\left(-2 \xi_{0} t, 2 \xi t\right) \in \operatorname{suppsing}(u)$. 


\section{Apêndice A}

\section{o lema de Morse}

Lema A.1 (Lema de Morse) Suponha que $\Phi$ tem um ponto crítico não degenerado na origem e que $\Phi(0)=0$. Então próximo de $y=0$ existe uma mudança de variáveis $C^{\infty}, y \rightarrow \widetilde{y}$, tal que nas novas coordenadas,

$$
\Phi(\widetilde{y})=1 / 2\left({\widetilde{y_{1}}}^{2}+\ldots+{\widetilde{y_{j}}}^{2}-{\widetilde{y_{j+1}}}^{2} \ldots-{\widetilde{y_{n}}}^{2}\right) .
$$

Prova: Depois de fazer uma mudança de variáveis (linear) inicial, podemos sempre assumir que

$$
\left(\frac{\partial^{2} \Phi}{\partial y_{j} \partial y_{k}}\right)=\left(\begin{array}{cccccc}
1 & 0 & 0 & 0 & 0 & \ldots \\
0 & \ddots & \vdots & \vdots & \vdots & 0 \\
0 & \ldots & 1 & 0 & \ldots & 0 \\
0 & \ldots & 0 & -1 & 0 & \ldots \\
0 & \vdots & 0 & 0 & \ddots & 0 \\
0 & 0 & 0 & 0 & \ldots & -1
\end{array}\right)
$$

quando $y=0$. Então, dado que $\frac{\partial \Phi}{\partial y_{1}}=0$ e $\frac{\partial^{2} \Phi}{\partial y_{1}^{2}} \neq 0$ na origem, segue do teorema da função implícita que deve existir uma função $\overline{y_{1}}=\overline{y_{1}}(y) \in C^{\infty}$ solução da equação

$$
\left(\frac{\partial \Phi\left(\overline{y_{1}}, y^{\prime}\right)}{\partial y_{1}}\right)=0
$$

Portanto, se fizermos a mudança de variáveis

$$
y \rightarrow\left(y_{1}-\overline{y_{1}}, y^{\prime}\right)
$$


sempre podemos assumir que $\overline{y_{1}}=0$, que é

$$
\left(\frac{\partial \Phi\left(0, y^{\prime}\right)}{\partial y_{1}}\right)=0
$$

mas, pelo teorema de Taylor, isto significa que estamos na situação onde

$$
\Phi(y)=\Phi\left(0, y^{\prime}\right)+C(y) y_{1}^{2},
$$

onde a função $C \in C^{\infty}$, a qual é positiva próxima da origem. Finalmente, se colocamos

$$
\widetilde{y_{1}}(y)=(C(y))^{1 / 2} y_{1},
$$

segue que

$$
\Phi(y)=1 / 2{\widetilde{y_{1}}}^{2}+\widetilde{\Phi}\left(y^{\prime}\right),
$$

o que, por indução, dá o lema A.1 de (Morse). 


\section{Apêndice B}

\section{Subespaços lagrangeanos}

Lema B.1 Se $V_{0}$ e $V_{1}$ são dois subespaços lagrangeanos de $T^{*}\left(\mathbb{R}^{n}\right)$ podemos achar um terceiro subespaço lagrangeano $V$, o qual é transverso a ambos $V_{0}$ e $V_{1}$.

Prova: É suficiente provar que podemos escolher uma base simplética $e_{1}, \ldots e_{n}$, $\epsilon_{1}, \ldots, \epsilon_{n}$ de $T^{*}\left(\mathbb{R}^{n}\right)$ tal que $V_{0}$ é estendida por $\epsilon_{1}, \ldots, \epsilon_{n}$ e $V_{1}$ por $e_{k+1}, \ldots e_{n}, \epsilon_{1}, \ldots, \epsilon_{k}$. Podemos então tomar $V$ como o subespaço

$$
\begin{aligned}
& V= \\
& \left\{\sum x_{j} e_{j}+\sum \xi_{j} \epsilon_{j}: x^{\prime \prime}=\xi^{\prime \prime}, \xi^{\prime}=0 \quad \text { com } \quad x^{\prime}=\left(x_{1}, \ldots, x_{k}\right), x^{\prime \prime}=\left(x_{k+1}, \ldots, x_{n}\right)\right\} .
\end{aligned}
$$

Para verificar esta afirmação escolhemos uma base $\epsilon_{1}, \ldots, \epsilon_{k}$ para o subespaço $V_{0} \cap V_{1}$ e estendemos isto para uma base total $\epsilon_{1}, \ldots, \epsilon_{n}$ de $V_{0}$. Se limitamos $\sigma$ para $V_{0} \times V_{1}$, vemos que isto dá um duplo entre $V_{0} \backslash\left(V_{0} \cap V_{1}\right)$ e $V_{1} \backslash\left(V_{0} \cap V_{1}\right)$. Isto acontece porque ambos $V_{0}$ e $V_{1}$ são lagrangeanos e daí $V_{j}^{\perp}=V_{j}, \quad j=0,1$. Por conta disto podemos escolher $\left(e_{k+1}, \ldots, e_{n}\right) \in V_{1}$ tal que $\sigma\left(\epsilon_{i}, e_{j}\right)=\delta_{i j}$ se $k+1 \leq i, \quad j \leq n$, se estendemos a base simplética parcial $\left\{e_{j}\right\}_{k+1}^{n}, \quad\left\{\epsilon_{j}\right\}_{1}^{n}$ para uma base simplética total, usando o teorema 4.1. 


\section{Apêndice C}

\section{Propriedades da função beta}

Observação: Vejamos outras propriedades de $\Gamma$ que precisamos no capitulo 1. Se $a>0$ e $b>0$

$$
B(a, b)=\int_{0}^{1} t^{a-1}(a-t)^{b-1} d t
$$

é chamada função beta. Além disso temos que $B(a, b)=\frac{\Gamma(a) \Gamma(b)}{\Gamma(a+b)}$. Tomando $t=1 /(1+s)$ como nova variável obtemos

$$
B(a, 1-a)=\int_{0}^{1} t^{a-1}(1-t)^{-a} d t=\int_{0}^{\infty} s^{-a}(1+s)^{-1} d s, \quad 0<a<1 .
$$

Integrando $s^{-a}(1+s)^{-1}$ em $\mathbb{C}$ e fazendo um corte ao longo de $\mathbb{R}_{+}$, primeiro de $\mathbb{R}-i 0$ a 0 , depois a $\mathbb{R}+i 0$ e finalmente ao longo do círculo $|s|=R$ a $\mathbb{R}-i 0$, obtemos, quando $\mathbb{R} \rightarrow \infty$,

$$
B(a, 1-a)\left(1-e^{-2 \pi i a}\right)=2 \pi i e^{-\pi i a},
$$

o que podemos escrever como

$$
\Gamma(a) \Gamma(1-a)=B(a, 1-a)=\pi / \sin (\pi a) .
$$

Por continuidade analítica, isto vale também quando $a$ não é inteiro. 


\section{Referências}

[1] Sogge C., Fourier integrals in classical analysis, Cambridge, 1993.

[2] Hörmander L. Duistermaat J.J., Guillemin V.W., Fourier integral operator.1, Mathematics past and present Fourier integral operator (Victor W. Guillemin Jochen Bruning, ed.), Springer-Verlag, 1971, pp. 23-182.

[3] Treves F., Introduction to pseudo-differential and fourier integral operators, vol. 2, Plenum, 1980.

[4] Hounie J., Teoria elementar das distribuições, 12 Colóquio Brasileiro de Matemáticas, IMPA, RJ-Brasil, 1979.

[5] _ Introdução aos operadores pseudo-diferenciais, 16 Colóquio Brasileiro de Matemáticas, IMPA, RJ-Brasil. 1987.

[6] Hörmander L., On the existence and the regularity of solution of linear pseudo-differential equations, Eiseignement Math (1971), 99-163.

[7] - Seminar on singularities of solutions of linear PDE (Princeton university press e university of tokyo press. eds.), no. 91, Annals of Mathematics studies, Princeton, New Jersey, 1979.

[8] _ The analysis of linear partial differential operators, vol. I, SpringerVerlag, Berlin, 1985.

[9] Nirenberg L., Lectures on linear partial differential equations, Americam Mathematical Society (1972), no. 17, 19-45.

[10] Shubin M.A., Pseudo-differential operators and spectral theory, SpringerVerlag, 1978.

[11] Cordaro P., Notas da disciplina teoria das distribuições e análise de fourier, USP, 1999. 
[12] Schirmer P.P., Tópicos em equacões diferenciais hiperbólicas, 21 Colóquio Brasileiro de Matemáticas, IMPA, RJ-Brasil, 1997. 


\section{Índice Remissivo}

índice, 88

base simpléctica, 67

bicaracterísticas, 86

complemento ortogonal simpléctico, 67

conjunto frente de ondas, 50

corolário Relações de Sokhotskii, 13

derivada distribuicional, 10

distribuição, 9

homogênea, 15

equação do eikonal, 86

espaço

Besov, 71

de Minkowski, 90

das distribuições lagrangianas, 72

vetorial simpléctico, 66

existência da parametriz elíptica, 44

fatorização, 45

forma quadrática Lorentziana, 19

forma simpléctica, 66

função

de Heaviside, 11

holomorfa, 12

função de fase, 23

não degenerada, 69
Funções de Bessel, 35

gráfico canônico, 77

hiperfície

característica, 83

não-característica, 83

integral oscilatória, 25

Lema

da fase não-estacionária, 32

de Schur, 46

de Van der corput, 34

soma de Borel, 41

métrica Lorentziano, 89

O problema de Cauchy, 82

operador

pseudo-diferencial, 40

adjunto, 43

de onda, 19

elíptico, 85

hiperbólico, 86

integral de Fourier, 76

parabólico, 86

propriamente suportado, 41

parametriz local, 91

parte principal, 83 
Parte principal da integral de Cauchy, 10

produto escalar Lorentziano, 89

relação canônica homogênea, 71

símbolos, 24

secção, 70

solução fundamental, 17

do operador de Laplace, 17

do operador de onda, 18

subespaço

lagrangiano transverso, 69

subespaço vetorial

coisotrópico, 67

isotrópico, 67

lagrangeano, 67

simpléctico, 67

subvariedade lagrangeana, 69

Superfícies características, 82

suporte singular, 9

\section{Teorema}

da composição de FIO, 78

da integral oscilatória, 25

Conjunto Frente de onda da integral oscilatória, 57

da parametriz local, 91

de Gauss no plano complexo, 12

do valor de fronteira de funções

holomorfas, 12

Solução fundamental do operador de onda, 19
Lorentziana, 89

simpléctica, 68

vetor

tipo espaço, 90

tipo luz, 90

tipo tempo, 90 